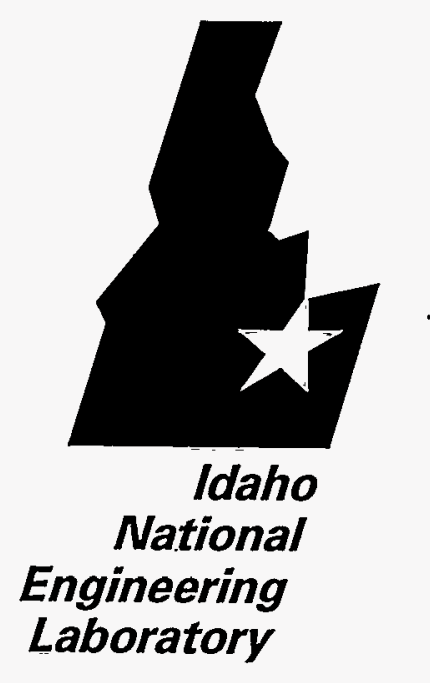

INEL-96/0076

Rev. 0

RECEIVED

June 1996

JUL 291996

OSTI

Use of Monte Carlo Methods in Environmental Risk Assessment at the INEL: Applications and Issues

Gerald Harris

Robin Van Horn

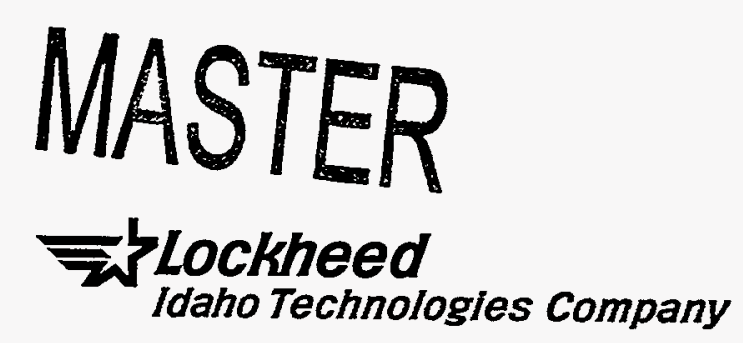

DISTRIBUTION OF THIS DOCUMENT IS UNLIMTEDD 


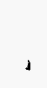




\section{Use of Monte Carlo Methods in Environmental Risk Assessments at the INEL: Applications and Issues}

Gerald Harris

Robin Van Horn

June 1996

\section{Idaho National Engineering Laboratory Environmental Restoration Department Lockheed Martin Idaho Technologies Company Idaho Falls, Idaho $\mathbf{8 3 4 1 5}$}

Prepared for the

U.S. Department of Energy

Assistant Secretary for Environmental Management

Under DOE Idaho Operations Office

Contract DE-AC07-94ID 13223 



\section{DISCLAIMER}

Portions of this document may be illegible in electronic image products. Images are produced from the best available original document. 
. 


\section{Use of Monte Carlo Methods in Environmental Risk Assessment at the INEL: Applications and Issues}

INEL-96/0076

Revision 0

June 1996

Approved by:

This report was prepared as an account of work sponsored by an agency of the United States Government. Neither the United States Government nor any agency thereof, nor any of their employees, makes any warranty, express or implied, or assumes any legal liability or responsibility for the accuracy, completeness, or usefulness of any information, apparatus, product, or process disclosed, or represents that its use would not infringe privately owned rights. Reference herein to any specific commercial product, process, or service by trade name, trademark, manufacturer, or otherwise does not necessarily constitute or imply its endorsement, recommendation, or favoring by the United States Government or any agency thereof. The views and opinions of authors expressed herein do not necessarily state or reflect those of the United States Government or any agency thereof. 



\begin{abstract}
The EPA is increasingly considering the use of probabilistic risk assessment techniques as an alternative or refinement of the current point estimate of risk. This report provides an overview of the probabilistic technique called Monte Carlo Analysis. Advantages and disadvantages of implementing a Monte Carlo analysis over a point estimate analysis for environmental risk assessment are discussed. The general methodology is provided along with an example of its implementation. A phased approach to risk analysis that allows iterative refinement of the risk estimates is recommended for use at the INEL.
\end{abstract}





\section{EXECUTIVE SUMMARY}

Monte Carlo Analysis (MCA) is a powerful tool for evaluating risks in the presence of uncertainty. The technique could be useful in future environmental risk assessment work done at the Idaho National Engineering Laboratory (INEL). This document provides the background information useful for risk assessors and risk managers to understand MCA. It presents the general methodology of MCA and identifies issues that will need to be resolved before MCA can be effectively implemented at the INEL.

MCA is a conceptually simple method of probabilistic risk assessment. For the input parameters in the risk model, a probability distribution is assigned based on available information about the parameter; these distributions are each randomly sampled $n$ times. The $n$ random realizations from each input parameter are then entered into the model to produce $n$ risk estimates. The input distributions take into account uncertainties in those parameters; the resulting risk values provide an estimate of the risk distribution. Hence, the Monte Carlo analysis essentially propagates the input uncertainties through the risk model to the resulting risk estimates.

In this report, the motivation for using MCA as an improvement over the baseline risk assessment (BRA) point estimates is given. Two major shortcomings of the point estimate approach is that it suffers from creeping conservatism so that the user does not know how conservative the estimate is, and it provides limited information to the risk manager and public. The use of MCA helps alleviate both of these problems.

The use of MCA helps focus attention on the uncertainty in the risk assessment. Further, it provides a quantitative description of the uncertainty. In this way, the manager and stakeholders are better able to understand the risks and are better able to make informed decisions. A key theme of the document is that uncertainty matters and MCA provides a practical description of the uncertainty.
Some reasons for implementing an $\mathrm{MCA}$ include:

- Evidence shows that "best estimates" are not particularly good estimates; the quality of the estimates is significantly improved when the analysis forces the consideration of uncertainties.

- One purpose of risk assessment is to help anticipate the unexpected; again, by explicitly considering uncertainties, it is easier to determine the contingencies.

- Risk managers must rely on experts when making decisions; without uncertainty analysis, the manager cannot assess the reliability of the expert's information.

Some of the disadvantages of implementing an MCA include:

- Adds increased complexity to the risk assessment process

- Difficult to determine a good MCA from a bad MCA

- Some skepticism of MCA by regulatory agencies

- More intensive data requirements than a BRA.

Except under the situation in which the point estimate of risk leads to an unequivocal decision for an INEL site, the advantages of implementing an MCA far outweigh the disadvantages. A phased approach to implementing MCA risk analysis is recommended. Each phase would be a refinement, based on more information, of the previous phase. Sensitivity and correlation results from each phase would be useful in identifying which parameters to focus effort on.

In general, the MCA will be most useful when a better understanding of the risks and the processes creating them is needed. Situations in which the 
MCA should be considered as a tool in the risk assessment include:

- When the calculated baseline point risks are - equivocal (say between $1 \times 10^{-6}$ and $1 \times 10^{-4}$ )

- When there are a large number of input parameters and exposure pathways subject to uncertainty.

While the actual implementation of MCA is straightforward, there are a number of issues that need to be agreed upon by all INEL stakeholders. These issues include selection of parameter distributions, use of expert judgment, implementation of correlation between parameters, and bounding of the assessment. Of these, selection of parameter distributions may be the most contentious.

When selecting parameter distributions, consideration must be given to the level of specificity and the source of the data. For some parameters and under certain scenarios, a distribution based on regional or national data may be adequate, while for other parameters or scenarios, the distribution must be site-specific. In any case, all stakeholders should agree to the use of the selected distributions.

A related issue is with the development of uncertainty distributions for the toxicity parameter. Toxicity values are subject to a great deal of uncertainty. They are generally derived from animal studies using much higher doses than would result from any possible INEL exposure. While models have been proposed for extrapolating high dose results to low dose and animal studies to humans, quantifying the uncertainties in these extrapolations are not obvious. As with the other input parameter distributions, all stakeholders should agree to the use of the selected toxicity distributions.

Prior to performing the MCA, the problem being considered must be well understood and defined (as with any risk assessment). The scenarios, pathways, receptors, and contaminants of interest must be clearly defined and agreed upon.
Once the problem has been well-formulated, there are four basic steps to implementing the Monte Carlo assessment.

1. Implement a sensitivity analysis of the risk, identifying parameters that most impact the risk estimate.

2. Determine appropriate probability density functions (PDFs) or cumulative distribution functions (CDFs) for parameters identified in Step 1.

3. Implement Monte Carlo sampling of input distributions to calculate the risk distribution.

- Determine the number of Monte Carlo samples, $n$, needed to get a reasonably precise estimate of the risk distribution.

- Assess the reproducibility and representativeness of the results. Also, implement a final sensitivity analysis showing which parameters have the largest impact on the overall uncertainty in the risk estimates.

4. Present a graphical summary of the results along with a qualitative discussion. Clearly identify the uncertainties that were quantified in the analysis. Discuss other qualitative uncertainties not included in the quantitative analysis. Present the results in such a manner that the significance of the risks are clearly understood by the decision-makers.

The efficiency of the Monte Carlo methodology may be improved by using Latin Hypercube Sampling (LHS). In LHS, each input parameter distribution is broken up into $n$ (the number of samples) equi-probable intervals and a value is selected from each interval. These $n$ values are then randomly matched with the $n$ values from the other distributions to form $n$ sets of input parameter. These sets are input to the model to generate $n$ risk values. This method ensures good coverage of each of the input parameters and speeds convergence of the tails of the risk distribution. 
While the MCA methodology provides a significantly better description of the risk than does a point estimate, it is not a panacea. It will require some conservative assumptions, though the degree of conservatism will be less than in the point estimate. MCA is subject to the same qualitative uncertainties as the point estimate, including that due to the model and scenarios considered.
MCA methodology is recommended as a tool beyond the BRA point estimate of risk. While this document provides a general framework and methodology for MCA, it is not a protocol. Currently WAG 7 is implementing a probabilistic risk assessment using MCA techniques. Some of the lessons learned from that experience will be invaluable in developing a more detailed protocol for the use of MCA at the INEL. 
viii 


\section{CONTENTS}

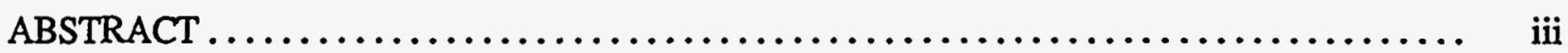

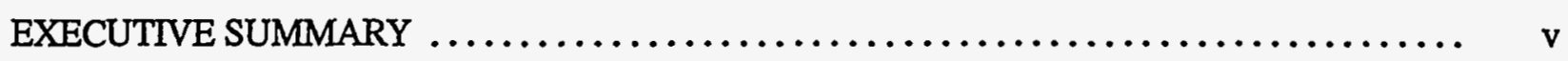

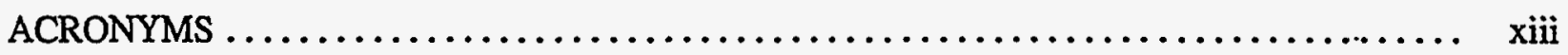

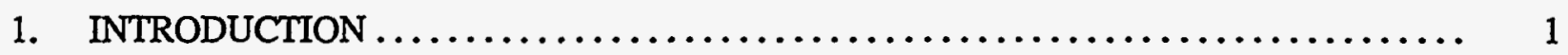

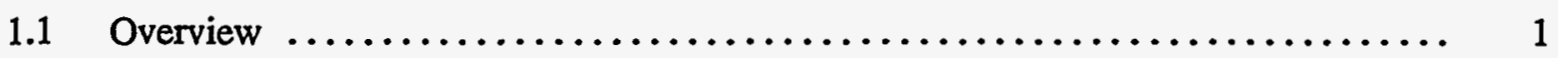

1.2 Risk, Risk Assessment, and Risk Management $\ldots \ldots \ldots \ldots \ldots \ldots \ldots \ldots \ldots . . \ldots \ldots$

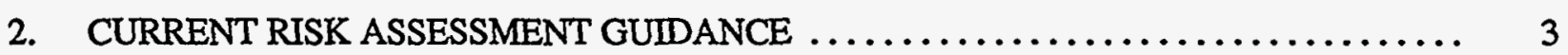

3. IMPORTANCE OF UNCERTAINTY ANALYSIS $\ldots \ldots \ldots \ldots \ldots \ldots \ldots \ldots \ldots \ldots$

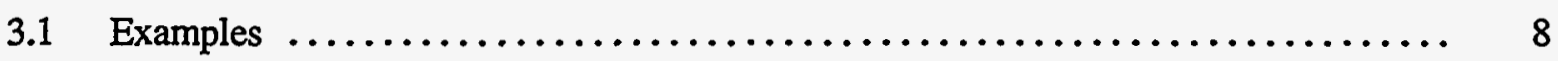

3.1.1 Example 1: Does the Uncertainty Wash Out? ................. 8

3.1.2 Example 2: The Limitation of the Point Estimate. ................ 8

3.1.3 Example 3: A More Complete and Informative Assessment............ 9

3.2 Possible Problems with Monte Carlo Analysis $\ldots \ldots \ldots \ldots \ldots \ldots \ldots \ldots \ldots . . \ldots \ldots$

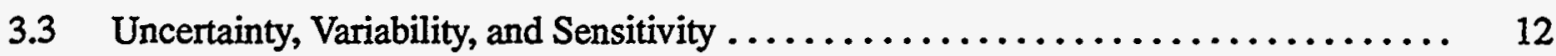

3.3.1 Uncertainty and Variability $\ldots \ldots \ldots \ldots \ldots \ldots \ldots \ldots \ldots \ldots, 12$

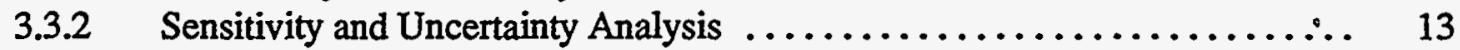

3.4 Summary $\ldots \ldots \ldots \ldots \ldots \ldots \ldots \ldots \ldots \ldots \ldots \ldots \ldots \ldots \ldots \ldots \ldots \ldots \ldots, 14$

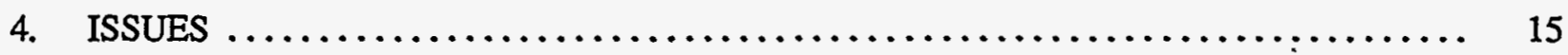

4.1 When to Implement a Monte Carlo Analysis ........................ 15

4.2 Level of Detail and Specificity ............................... 16

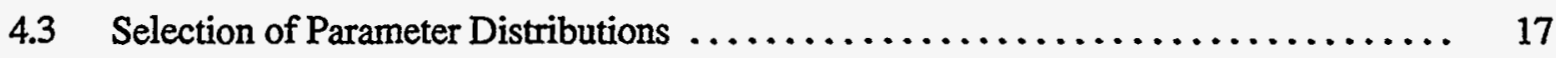

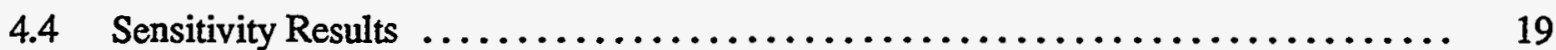

4.5 Correlation Between Parameter Uncertainty Distributions ................ 20

4.6 Expert Opinion $\ldots \ldots \ldots \ldots \ldots \ldots \ldots \ldots \ldots \ldots \ldots \ldots \ldots \ldots \ldots \ldots \ldots \ldots \ldots \ldots \ldots \ldots \ldots \ldots, 21$

4.7 Bounding the Assessment/Problem $\ldots \ldots \ldots \ldots \ldots \ldots \ldots \ldots \ldots \ldots \ldots \ldots, 21$

$4.8 \quad$ Ecological Risk Assessment $\ldots \ldots \ldots \ldots \ldots \ldots \ldots \ldots \ldots \ldots \ldots \ldots \ldots \ldots, 22$

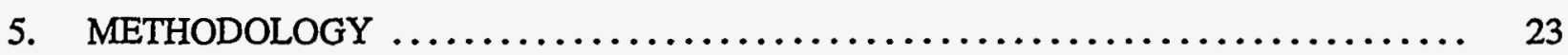

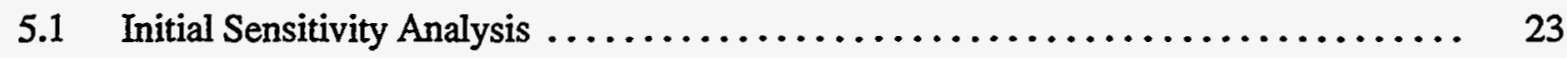


5.1.1 Nominal Range Method $\ldots \ldots \ldots \ldots \ldots \ldots \ldots \ldots \ldots \ldots \ldots \ldots, 23$

5.1.2 Some Caution Is Required $\ldots \ldots \ldots \ldots \ldots \ldots \ldots \ldots \ldots \ldots \ldots \ldots \ldots \ldots \ldots \ldots, 24$

5.2 Distribution Selection $\ldots \ldots \ldots \ldots \ldots \ldots \ldots \ldots \ldots \ldots \ldots \ldots \ldots \ldots, 24$

5.2.1 Types of Quantities $\ldots \ldots \ldots \ldots \ldots \ldots \ldots \ldots \ldots \ldots \ldots \ldots \ldots \ldots \ldots \ldots \ldots \ldots \ldots, 24$

5.2 .2 Types of Distributions $\ldots \ldots \ldots \ldots \ldots \ldots \ldots \ldots \ldots \ldots \ldots \ldots \ldots \ldots \ldots \ldots \ldots, 27$

5.2.3 Selecting Distributions $\ldots \ldots \ldots \ldots \ldots \ldots \ldots \ldots \ldots \ldots \ldots \ldots \ldots, \quad 33$

$5.3 \quad$ Monte Carlo Sampling $\ldots \ldots \ldots \ldots \ldots \ldots \ldots \ldots \ldots \ldots \ldots \ldots \ldots \ldots \ldots \ldots \ldots \ldots \ldots \ldots, 34$

5.3.1 Determine the Number of Monte Carlo Samples ................. 34

5.3.2 Assess Quality of the Results ........................... 36

5.3.3 Implementation of the Monte Carlo Simulation ................. 38

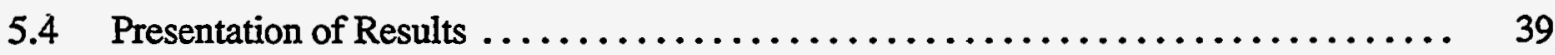

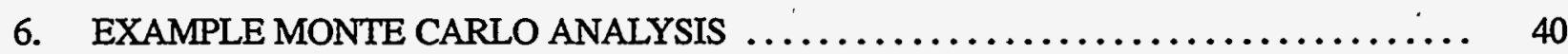

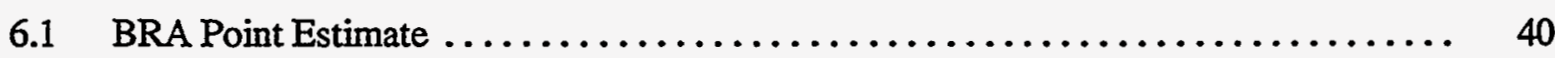

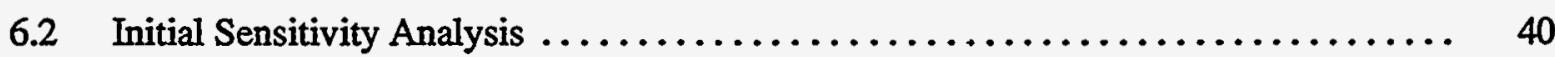

$6.3 \quad$ Parameter Distribution Selection $\ldots \ldots \ldots \ldots \ldots \ldots \ldots \ldots \ldots \ldots \ldots \ldots, 42$

6.3.1 Benzene Concentration in Soil $\ldots \ldots \ldots \ldots \ldots \ldots \ldots \ldots \ldots \ldots \ldots \ldots, 43$

6.3.2 Exposure Duration $\ldots \ldots \ldots \ldots \ldots \ldots \ldots \ldots \ldots \ldots \ldots \ldots \ldots \ldots, 44$

6.3.3 Ingestion Rate $\ldots \ldots \ldots \ldots \ldots \ldots \ldots \ldots \ldots \ldots \ldots \ldots \ldots \ldots \ldots, \quad 45$

6.3.4 Slope Factor $\ldots \ldots \ldots \ldots \ldots \ldots \ldots \ldots \ldots \ldots \ldots \ldots \ldots \ldots \ldots, \quad 47$

6.3.5 Averaging Time Distribution $\ldots \ldots \ldots \ldots \ldots \ldots \ldots \ldots \ldots \ldots \ldots \ldots, \quad 47$

6.3.6 Body Weight $\ldots \ldots \ldots \ldots \ldots \ldots \ldots \ldots \ldots \ldots \ldots \ldots \ldots \ldots \ldots, 50$

6.4 Number of Monte Carlo Samples ............................ 50

6.5 Monte Carlo Implementation $\ldots \ldots \ldots \ldots \ldots \ldots \ldots \ldots \ldots \ldots \ldots \ldots \ldots \ldots, 50$

6.6 Quality of the Results $\ldots \ldots \ldots \ldots \ldots \ldots \ldots \ldots \ldots \ldots \ldots \ldots \ldots \ldots \ldots \ldots \ldots \ldots \ldots, \quad 52$

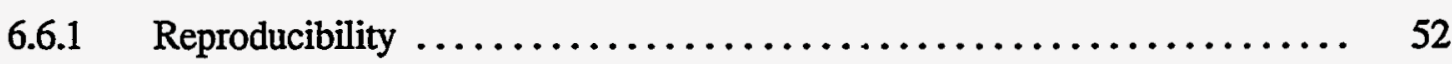

6.6.2 Sensitivity of Risk to Input Parameters $\ldots \ldots \ldots \ldots \ldots \ldots \ldots \ldots \ldots \ldots . \quad 53$

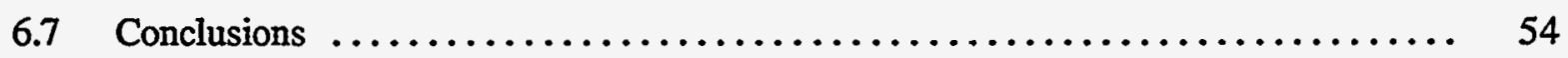

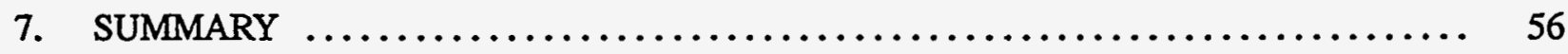

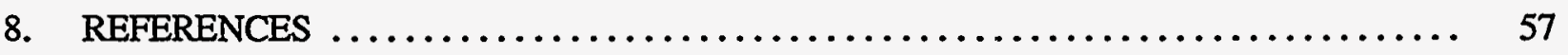




\section{FIGURES}

1. Plot of the reduction efficiencies of the two soil remediation methods $\ldots \ldots \ldots \ldots \ldots \ldots \ldots$

2. Plot of the simulated cancer risk distributions $\ldots \ldots \ldots \ldots \ldots \ldots \ldots \ldots \ldots \ldots \ldots \ldots \ldots$

3. Plots of the density or mass functions for common distribution types .............. 29

4. Example of LHS, with two variables and three samples $\ldots \ldots \ldots \ldots \ldots \ldots \ldots \ldots$

5. Fit of a lognormal distribution to benzene concentration in soil $\ldots \ldots \ldots \ldots \ldots \ldots \ldots \ldots$

6. Probability density function for benzene concentration in soil $\ldots \ldots \ldots \ldots \ldots \ldots \ldots \ldots$

7. Exposure duration (total residence time) distribution fit $\ldots \ldots \ldots \ldots \ldots \ldots \ldots \ldots \ldots$

8. Probability density function for exposure duration $\ldots \ldots \ldots \ldots \ldots \ldots \ldots \ldots \ldots \ldots \ldots$

9. Probability density function for soil ingestion rate $\ldots \ldots \ldots \ldots \ldots \ldots \ldots \ldots \ldots \ldots$

10. Probability density function for slope factor $\ldots \ldots \ldots \ldots \ldots \ldots \ldots \ldots \ldots \ldots \ldots$

11. Averaging time (age) distribution fit $\ldots \ldots \ldots \ldots \ldots \ldots \ldots \ldots \ldots \ldots \ldots \ldots \ldots \ldots$

12. Probability density function for averaging time $\ldots \ldots \ldots \ldots \ldots \ldots \ldots \ldots \ldots \ldots \ldots$

13. Probability density function for body weight $\ldots \ldots \ldots \ldots \ldots \ldots \ldots \ldots \ldots \ldots \ldots \ldots$

14. Cumulative distribution function of risks as observed from the Monte Carlo simulations .... 51

15. Probability density function of risks as observed from the Monte Carlo simulations ...... 52

\section{TABLES}

1. Risk equation parameters for which enough data are available to develop standard probability density functions (from Finley and Paustenbach 1994) .............. 18

2. Sensitive and uncertain parameters in INEL risk assessments (Smith 1994) ......... 20

3. Summary of types of quantities used in risk/exposure equations (Morgan and Henrion 1990) ... 25

4. Standard probability density functions proposed by Brorby and Finley (1994) ........ 35

5. Variables and constants used in the benzene-contaminated soil ingestion scenario ........ 41

6. Input values and calculated risks for the nominal range method sensitivity analysis ...... 41

7. Rank of parameter importance in the risk equation as determined by the nominal range method .......................................... 42

8. Summary statistics for results of the Monte Carlo analysis $\ldots \ldots \ldots \ldots \ldots \ldots \ldots \ldots \ldots$ 
9. Comparison of risk summary statistics between the original simulation and its replicate .....

10. Comparison of percentiles of the risk distributions between the original simulation and its replicate

11. Measures of influence of the input parameters on the risk as observed in the original simulation and its replicate 


\section{ACRONYMS}

BRA baseline risk assessment

$\mathrm{CDF}$ cumulative distribution function

DOE U.S. Department of Energy

EPA U.S. Environmental Protection Agency

HEAST Health Effects Assessment Summary Tables

HI hazard index

INEL Idaho National Engineering Laboratory

IRIS Integrated Risk Information System

MCA Monte Carlo analysis

NRC U.S. Nuclear Regulatory Commission
PBPK Physiologically-Based Pharmacokinetic

PDF probability density function

PMF probability mass function

PRA probabilistic risk assessment

RAGS Risk Assessment Guidance for Superfund

RfD reference dose

RI/FS remedial investigation/feasibility study

RME reasonable maximum exposure

SF slope factor

TUBE theoretical upper bound estimate 


\section{Use of Monte Carlo Methods in Environmental Risk Assessment at the INEL: Applications and Issues}

\section{INTRODUCTION}

Monte Carlo analysis (MCA) is an increasingly popular tool in human health and ecological risk assessment that uses distributions rather than single point estimates to represent input parameters (e.g., body weight, ingestion rate) to produce a distribution of risks. Human health risk assessments commonly use baseline risk assessment (BRA) methodology. In the performance of a BRA, the analysis typically uses conservative assumptions to determine a single point estimate of risk. For certain sites or Waste Area Groups (WAGs) at the Idaho National Engineering Laboratory (INEL), it may be necessary and desirable to have more information about the possible risk for the decision-making process than a point estimate. As a next step, MCA

- Allows for explicit quantitative assessment of the uncertainty in the risk analysis

- Provides more information to the risk manager

- Alleviates the need for conservatism.

MCA is one of a variety of probabilistic methods in risk assessment. In the field of human health and ecological risk assessment, the terms "probabilistic risk assessment" (PRA) and Monte Carlo analysis are sometimes used interchangeably, however this report retains the distinction that MCA comprises a subset of the PRA methods. The U.S. Environmental Protection Agency's (EPA's) guidelines for risk assessment use fairly simple models of exposure and risk. Because there is uncertainty in the input model parameters, the resulting risk or exposure estimates are subject to the propagation of these uncertainties. MCA provides a probabilistic approach to propagating these input uncertainties to better characterize the resulting risk and exposure estimates. It does so by the repeated random sampling of the input parameter distributions to produce an output (risk or exposure) distribution.

The advantages of the probabilistic assessment (and MCA, in particular) of risks and exposures over the point estimate approach are as follows (Finley and Paustenbach 1994):

- Provides more meaningful information to risk managers and the public

- Avoids disputes over best point estimates

- Associates risk estimates with a quantitative measure of uncertainty

- Reduces creeping conservatism

- Allows for quantitative evaluation of conservatism in the point estimate approach

- Provides a more meaningful sensitivity analysis.

This report discusses some of these advantages in more detail, along with some potential problems associated with the use of MCA. Additionally, the report provides a framework for the implementation of MCAs at the INEL.

\subsection{Overview}

This report serves as an introduction to MCA. It provides a general overview of PRA, and focuses on the use of Monte Carlo sampling and related methods. The document does assume a certain degree of knowledge of the current environmental risk assessment methods used at the INEL. The report is expected to be most useful to those who need a good understanding of Monte Carlo methods. This may include both technical and managerial people. There is a large body of literature on 
PRA and MCA; this report focuses on what has been produced for environmental risk assessment.

One of the "nice" aspects of Monte Carlo sampling is that it provides a conceptually simple method of uncertainty analysis. However, technical details and issues identified in this report will need resolution and probably agreement upon by all risk assessment stakeholders at the INEL. Also, since point estimates of risk are also conceptually simple, part of the report establishes what is to be gained by using a Monte Carlo analysis of risk.

The remainder of this report is divided into several broad topics. The first, comprising Section 2, reviews the BRA guidance and practices as they relate to the INEL. The second, comprising Section 3, discusses uncertainty and its relevance in risk assessment. The third, comprising Section 4, identifies issues in MCA for which there is no general consensus on what constitutes best practice. Recommendations for handling these issues are made with the understanding that all stakeholders agree and understand the consequences of the recommendations. The fourth, comprising Section 5, provides a detailed description of the MCA methodology. These sections provide sufficient detail that a risk analyst would likely be able to implement an MCA. Because this report advocates an iterative approach to MCA, no explicit cookbook methodology is given. Finally, Section 6 presents an annotated example of a simple MCA analysis.

\subsection{Risk, Risk Assessment, and Risk Management}

Risk is the possibility of suffering harm or loss. This dictionary definition contains two key components of risk: risk only refers to negative consequences, and it has an element of uncertainty. A more formal and quantitative definition given by Kaplan and Garrick (1981) as the set of all possible triplets ( event, probability of the event, consequence of the event $\}$ ) from which one can derive the expected adverse consequence. Probability is considered to represent subjective degrees of belief as opposed to the classical frequentist definition.

Risk analysis/risk assessment should explicitly do five things:

1. Identify scenarios that can lead to loss or harm

2. Identify the loss or harm that may result

3. Estimate the likelihood of such loss or harm occurring

4. Discuss the uncertainties in the estimates

5. Clearly communicate the significance of the results to the decision-makers/risk managers.

Risk management uses the results of the risk assessment along with other social, political, ethical, and financial considerations to come to decisions. The uncertainties in the risk assessment must be factored into the decision process of the risk manager to ensure a result that balances all considerations.

The point estimate approach used for assessing environmental and human health risks (and management decisions) at the INEL uses a qualitative discussion of uncertainty. This approach assumes that the uncertainty inherent in the risk estimate can be overcome by making conservative assumptions so as to be protective of human health and the environment. This tends to result in conservative, but hopefully not unrealistic, point estimates. Since the final point estimate tends to be what is reported or considered, publicly, it is subject to misinterpretation. The impact of the uncertainty on the estimate is nebulous in the decision-making process. A central theme of this report is that uncertainty does matter, and that MCA is a convenient method for quantifying that uncertainty so that it is more integral to the decision-making process. 


\section{CURRENT RISK ASSESSMENT GUIDANCE}

The Federal Facilities Agreement/Consent Order (FFA/CO) and associated Action Plan (AP) describe the governance and implementation of remediation of the INEL and dictate the use of BRA in the process. The AP provides the schedules for all INEL operable units records of decisions. The critical-path schedule is based on the following conditions:

1. The last Track 2 Summary Report for each WAG will be submitted before submittal of the last RI/FS scope of work for that WAG

2. All Track 1 reviews for each WAG will be completed before the submittal of the last Track 2 Sampling and Analysis Plan for that WAG.

The RI/FS process includes development and submittal of a draft $R I$ report with a complete BRA.

The current guidance for performance of a BRA is provided in the EPA's "Risk Assessment Guidance for Superfund Volumes I and II" (EPA 1989a), also known as RAGS. RAGS provides guidance on estimating the risk to humans from chemical exposures. The methodology presented in RAGS is a baseline risk assessment because it assumes no remediation or institutional controls are applied to the site. The methodology has four basic steps:

1. Data collection and evaluation

- Gather and analyze relevant site data

- Identify potential chemical of concern.

\section{Exposure assessment}

- Analyze contaminant releases

- Identify exposed populations

- Identify potential exposure pathways
- Estimate exposure concentrations for pathways

- Estimate contaminant intakes for pathways.

3. Toxicity assessment

- Collect qualitative and quantitative toxicity information

- Determine appropriate toxicity values.

4. Risk Characterization

- Characterize potential for adverse health effects to occur:

- Estimate cancer risks

- Estimate noncancer hazard quotients

- Evaluate uncertainty

- Summarize risk information.

The exposure estimate results in a measure called the "reasonable maximum exposure" (RME). The RME is the maximum exposure that is reasonably expected to occur at a site. RMEs are used so that the uncertainty is essentially overshadowed by the conservatism. The EPA recognizes that there is a great deal of uncertainty in environmental risk assessment; rather than quantifying the uncertainty, the RME approach biases the result to a high-end risk, thus protecting human health. The bias is expected to be large enough to account for the uncertainty. This is a reasonable approach for screening out from further consideration sites whose conservative risk estimate does not exceed the $10^{-6}$ to $10^{-4}$ range (these numbers indicate the probability that an individual within the exposed population will suffer harm, typically cancer, from exposure to the contaminant(s)). RAGS states that

Each intake variable in the equation has a range of values. For Superfund exposure assessments, intake variable values for a given pathway should be selected so that 
the combination of all intake variables results in an estimate of the reasonable maximum exposure for that pathway ... the reasonable maximum exposure (RME) is the maximum exposure that is reasonably expected to occur at a site. Under this approach, some intake variables may not be at their individual maximum values, but when in combination with other variables will result in estimates of RME. (pp. 6-19, emphasis in the original)

Due in part to concerns about the vagueness of the definition of the RME and its associated conservativeness, EPA (1992a) introduced a different approach for evaluating exposures. It defines three levels of exposure, the theoretical upper bounding estimate (TUBE), the high-end estimate, and the "typical" exposed individual. The TUBE is an upper limit on the exposure, beyond which no higher exposure can theoretically occur. The TUBE can be used to screen out various risk scenarios. The high-end exposed individual is the exposure/risk above the 90 th percentile of the population distribution of individual risks, but not higher than the individual in the population who has the highest risk. Note that the high-end risk is more a conceptual definition as given by the EPA (1992a). The typical exposed individual essentially represents the median exposure/risk.

The point estimate approach has a number of disadvantages. Finley and Paustenbach (1994) cite four disadvantages:

1. Repeated use of conservative point estimates tends to significantly overestimate actual exposure

2. Limited information for risk managers and public is provided

3. No associated measure of confidence is provided

4. Sensitivity or uncertainty analyses usually not very meaningful.
Nevertheless, the point estimate does have some advantages. It is generally simple to calculate. It is universally accepted by regulators. And it does allow screening of chemicals/pathways that are not significant risk drivers.

Recent guidelines for exposure assessment (EPA 1992a) recognize the value of determining distributions of exposures or doses and using Monte Carlo methods. The guidelines state that "exposure and dose information needs to be put in the appropriate form. Ideally, this would be a distribution of doses of the appropriate type across the population or population subgroup of interest." Further, in determining the distribution of doses, the guidelines state that "If data on the distribution of doses are not available, but data on the parameters used to calculate the dose are available, a simulation (such as an exposure model or Monte Carlo simulation) can sometimes be made of the distribution."

These guidelines (EPA 1992a) also recognize a need for better uncertainty analysis. They distinguish between uncertainty characterization (a qualitative discussion of the thought processes that lead to the selection and rejection of specific data, estimates, scenarios, etc.) and uncertainty assessment (a quantitative analysis of the uncertainty). They identify three broad categories of uncertainty:

1. Scenario uncertainty: Uncertainty regarding missing or incomplete information that is needed to fully define the exposure and dose.

2. Parameter uncertainty: Uncertainty regarding some parameter.

3. Model uncertainty: Uncertainty regarding gaps in scientific theory required to make predictions on the basis of causal inferences.

Scenario uncertainty and model uncertainty are difficult to assess quantitatively, and hence tend to be relegated to a qualitative analysis in the risk assessment. Rish (1988) discusses three approaches for analyzing model uncertainty: (1) validation of models, (2) verification of models, and (3) analysis of credible alternative models. Validation determines whether the model is representative of the phenomena being simulated. 
Verification determines if the computer code accurately performs the underlying model's calculations.

Parameter uncertainty is the area where most work has been done in developing quantitative methods. The guidelines (EPA 1992a) identify four levels of assessing uncertainty in the risk/ exposure estimates in increasing order of complexity and data requirements:

1. Sensitivity analysis: determine the effect of changes to a parameter's value on the exposure/dose.

2. Analytical uncertainty propagation: determine how the combined uncertainty and variability of the parameters produces the uncertainty and variability in the exposure/dose through use of mathematical derivations of variance propagations.

3. Probabilistic uncertainty analysis: determine how the combined uncertainty and variability of the parameters produces the uncertainty and variability in the exposure/dose through use of simulation. The Monte Carlo analysis assigns probability density functions (PDFs) to each parameter, PDFs repeatedly randomly selects values from each parameter's PDF, then enters these values into the risk/ exposure model to produce a distribution of exposure/dose.
4. Classical statistical methods: develop exposure/dose distributions directly from samples of exposures/doses. Generally, these methods can only be used for current risk scenarios which have measurable (as opposed to modeled) exposures/doses.

The DOE released an Information Brief on Guidelines for Exposure Assessment (DOE 1994a) that addresses the role of uncertainty analysis in the exposure assessment. It reiterates the discussion on the categories of uncertainty presented in the EPA guidelines (EPA 1992a). The information brief distinguishes between the results obtained from an uncertainty characterization and an uncertainty assessment. The distinction between the two methods is primarily the degree of sophistication in describing the uncertainty. For simple exposure assessment, uncertainty characterization (a qualitative discussion) may be all that is necessary. The uncertainty assessment is more quantitative and begins with simple measures and techniques and progresses to more complex methods as necessary.

In a companion Information Brief on Risk Characterization (DOE 1994b), the potential changes in the new guidance are discussed. The new guidance moves away from the RME to a high-end risk using a 90th percentile of the risk distribution. The use of Monte Carlo simulation for estimating risk/ exposure distributions is suggested in the new guidelines. 


\section{IMPORTANCE OF UNCERTAINTY ANALYSIS}

The need for uncertainty analysis and PRA has historically come about from the field of policy analysis and decision analysis. Much of the work developed from cost-benefit analyses of a variety of policy issues by a small number of think-tanks. Probabilistic risk assessment came to the attention of the nuclear power policy field with the publication of the Reactor Safety Study, NUREG-75/014 (WASH-1400), of 1975. This report formally handled uncertainty. While the report did have some technical faults, it led to the adoption of PRA methods in assessing reactor risks, as documented in the PRA Procedures Guide (NUREG/CR-2300, 1983).

While the EPA has been slower to adopt methods for handling uncertainties in risk assessments, it is being pressured towards more formal methods for handling uncertainties. In 1993, the EPA's Science Advisory Board issued a report (EPA 1993) recommending

that the EPA move to a distributional approach for calculating the RME, i.e., develop distributions for each of the terms or variables needed to calculate individual exposures and their distributions. These distributions determine a subsequent distribution for exposure, which can be calculated using Monte Carlo methods.

Some of the pressure towards change in the use of probabilistic methods is coming from Congress. For example, in a 1994 bill introduced in the House of Representatives (H.R. 4306, "Risk Assessment Improvement Act of 1994"), the Congress finds that "uncertainties inherent in risk assessments are neither adequately communicated by risk assessors nor clearly recognized by decision-makers and the public." One purpose of this Act is to establish a risk assessment program in the EPA that "develops risk characterization guidance and oversees its implementation in order to communicate the full range of risks and uncertainties" (emphasis added).
The EPA previously circumvented the problem of uncertainty by using RMEs. Virtually all parameters in risk equations were biased such that the resulting risk was higher than the true risk is believed to be. As discussed earlier, this can lead to unrealistic risk estimates. It is also difficult to defend what constitutes a reasonable maximum or worst-case scenario. Morgan and Henrion (1990) indicate an even more insidious problem with point estimates:

"There is considerable evidence to suggest that, because of the limitations of the human thought process, cognitive biases may give rise to "best estimates" that are not actually very good. Even if all that is needed is a "best estimate" answer, the quality of that answer may be significantly improved by an analysis that forces people to think about the full range of uncertainty associated with the problem."

Rish (1988) cites several reasons why uncertainties must be considered in risk analyses:

- The EPA has a responsibility to providethrough its regulations, guidelines, practices, and rulings-a reasonable level of assurance that protection of human health and the environment are maintained. In order to have confidence that this goal is achieved, the implications of uncertainties on regulatory decisions must be carefully assessed.

- There can be considerable costs associated with a decision based upon analysis with a high level of inherent uncertainty. These potential costs come from adopting a course of action which results in unexpected negative consequences, misplaced or practically irreversible commitments of resources, or policies which are difficult to alter at a later date when new information becomes available. Analysis of uncertainties can help to identify a risk management strategy which is most flexible to uncertain or changing conditions, and can provide a higher degree of confidence that risk management goals will be achieved. 
- Environmental risk analysis results can be highly sensitive to uncertainties in inputs or model formulations. Once the sources of uncertainties in the assessment are identified, their relative contribution to the overall uncertainty in risk estimates can be examined. This is useful information for planning measurement and modeling activities.

- When there is disagreement among sources of information, a good decision requires knowing the extent to which the disagreement would affect risk analysis results. An example would be disagreement among health experts about dose-response relationships.

- Many technological risk management problems involve complex mixtures of technical fact and value judgments. Explicit characterization of uncertainties can help to distinguish disagreements over technical uncertainties from those which are due to divergent values.

- The act itself of examining uncertainties in a quantitative manner results in a broader understanding of the processes being modelled, and the sources and nature of the controversial issues involved. It forces a careful review and characterization of the present state of knowledge, and it provides a structure for updating the risk assessment as information and understanding evolve.

In addition, Morgan and Henrion (1990) cite other reasons for uncertainty analysis in quantitative risk and policy analysis:

- A central purpose of policy research and policy analysis is to help identify the important factors and the sources of disagreement in a problem, and to help anticipate the unexpected. An explicit treatment of uncertainty forces us to think more carefully about such matters, helps us identify which factors are most and least important, and helps us plan for contingencies to hedge our bets.
- Increasingly we must rely on experts when we make decisions. It is often hard to be sure we understand exactly what they are telling us. It is harder still to know what to do when different experts appear to be telling us different things. If we insist they tell us about the uncertainty of their judgments, we will be clearer about how much they think they know and whether they really disagree.

- Rarely is any problem solved once and for all. Problems have a way of resurfacing. The details may change but the basic problems keep coming back again and again. Sometimes we would like to be able to use, or adapt, policy analyses that have been done in the past to help with the problems of the moment. This is much easier to do when the uncertainties of the past work have been carefully described, because then we can have greater confidence that we are using the earlier work in an appropriate way.

- Many real-world “decisions" are not made by a single person at a discrete time. More typically, a decision process may involve multiple actors making explicit and implicit decisions over an extended period. A piece of analysis will be more useful if it treats the uncertainty explicitly, allowing users to evaluate its conclusions and limitations better in the changing context of the ongoing decision process.

- Policy analysts have a professional and ethical responsibility to present not just "answers" but also a clear and explicit statement of the implications and limitations of their work. Attempts to fully characterize and deal with important associated uncertainties help them to execute this responsibility better.

Finkel (1990) notes additional reasons for implementing uncertainty analysis in risk assessments:

- Cost/benefit decisions should hinge on explicit comparisons of the probabilities and social costs associated with the possible true 
values of risk, not just a single comparison for one such value.

- Risk managers should avoid ranking different risks simply by comparing point estimates. He argues that comparing point estimates is extremely unreliable, particularly when comparisons become precarious because the degree of conservatism is hidden.

This is not to say that one can carelessly compare results from Monte Carlo analyses. However, in MCA, issues of comparability are more likely to be well characterized, while in the BRA the degree of conservatism and other factors that can affect comparability are often unknown or not well described.

The following examples are presented to support the premise that uncertainty analysis is a critical part of the decision-making process. These examples should develop an appreciation and better understanding of the role of uncertainty analysis in risk assessment.

\subsection{Examples}

The following examples illustrate the need for uncertainty assessments. Each example contrasts a point estimate with a result that accounts for uncertainty. These are brief examples focusing on matters of uncertainty rather than the methods used to get to the results. Section 6 provides a detailed example of a PRA.

\subsubsection{Example 1: Does the Uncertainty} Wash Out? Hoffman and Hammonds (1992) describe a lake contaminated with methyl mercury and the surrounding sediments contaminated with inorganic mercury. The upper $95 \%$ confidence limit of the concentration of methyl mercury in lake fish was $0.3 \mathrm{mg} / \mathrm{kg}$. The corresponding Hazard Index (HI) point estimate of risk to the maximally exposed individual (from ingestion of fish) is 0.94 . For the soil ingestion route, the upper 95\% confidence limit of the concentration of inorganic mercury in soil is $700 \mathrm{mg} / \mathrm{kg}$, corresponding to a $\mathrm{HI}$ point estimate of risk of 2.33 . Hence, it appears that the soil presents the greatest risk.
However, after taking into account the uncertainty in the input parameters and running a Monte Carlo analysis, Hoffman and Hammonds (1992) came to exactly the opposite conclusion. The $95 \%$ upper confidence limit on the HI for the soil ingestion pathway was 0.7 . The $95 \%$ upper confidence limit on the $\mathrm{HI}$ for the fish ingestion pathway was 1.2. They attribute this reversal to the large uncertainty in the Reference Dose (RfD) for inorganic mercury. While one would typically not compare a single percentile under a Monte Carlo analysis, the implication of this example is that the uncertainty in the RfD was not adequately described in the quantitative BRA point estimate.

In this example, the uncertainty in the two estimates was not equivalent and did not "wash out." That is, the conservatism in the fish ingestion scenario was not sufficient to account for the uncertainty in the RfD for inorganic mercury. Had the risk manager ignored the uncertainty, the best decision on how to act at this site may not have been made. Armed with the results of the uncertainty analy'sis, the manager is better equipped to make a sound decision.

Similar situations may occur at the INEL. In addition to uncertainties in contaminant toxicity estimates, some areas have a large variation in contaminant concentrations that, when taken into account in the risk assessment, could lead to similar reversals of decisions.

\subsubsection{Example 2: The Limitation of the Point Estimate. The point estimate provides} limited information, particularly in view of its weight in risk decisions. It is not clear exactly what the point estimate represents beyond a vague notion of a conservative estimate and "reasonable maximum exposure." Burmaster and Harris (1993) cite evidence that the EPA intends for the point estimate of risk not be exceeded by more than 5-10\% of the exposed population. The EPA (1992a) states, with qualifiers, that the high-end risks are risks "above the 90 th percentile of the population distribution, but not higher than the individual in the population who has the highest risk."

Even the EPA, in its 1992 guidelines for exposure assessment (EPA 1992a) recognize the 
limitations of the TUBE risk estimates. They note that the estimate is useful for eliminating sites from further consideration (if the estimate is less than the regulatory threshold). However, the bounding estimate of risk or exposure "cannot be used to make a determination that a pathway is significant . . . and it certainly cannot be used for an estimate of actual exposure (since by definition it is clearly outside the actual distribution)" (EPA 1992a).

So when the risk analyst presents a BRA point estimate of $5 \times 10^{-5}$, what can be concluded? From the above discussion, it could be concluded that no more than $10 \%$ of the exposed population exceeds this likelihood of getting cancer, and the person with the maximum risk has at least this risk level.

With an MCA, however, the risk analyst will be able to make more definitive statements. For example, an MCA allows conclusions such as: the risk estimate of $5 \times 10^{-5}$ represents the 95 th percentile of the population risk distribution, and that $1 \%$ of the population are actually subject to risks exceeding $1 \times 10^{-3}$, or that $99 \%$ of the population does not exceed the $1 \times 10^{-6}$ risk level, etc.

Hence, a decision based solely on the point estimate are inherently based on more nebulous information than the MCA results. The additional information provided by the MCA results allows the risk manager to make more reasoned and defensible decisions.

\subsubsection{Example 3: A More Complete and Informative Assessment. While an MCA is not a panacea for all risk management decisions, it will improve the manager's ability to better weigh the costs and benefits of various alternatives. The following is a simplified analysis that takes uncer- tainty into account when considering alternative remediation technologies.}

The manager of a site needs to choose between two remediation alternatives for cleaning up soil contaminated by carbon tetrachloride. The average soil concentration is $500 \mathrm{mg} / \mathrm{kg}$. A point estimate of the risk is calculated to be $5 \times 10^{-5}$. The manager has been told to reduce the risk to the $1 \times 10^{-6}$ level by reducing the average soil concentration to
$10 \mathrm{mg} / \mathrm{kg}$ carbon tetrachloride. Two technologies are available for the remediation. The first method, Method A, provides an $85 \%$ reduction in concentration at $\$ 1,000$ per ton processed. The second method, Method B, provides $95 \%$ reduction in concentration at $\$ 3,000$ per ton processed.

The manager notes that Method $A$ will reduce the concentration to about $75 \mathrm{mg} / \mathrm{kg}$ and Method B to $25 \mathrm{mg} / \mathrm{kg}$. While neither method reduces the soil concentration to below $10 \mathrm{mg} / \mathrm{kg}$, Method B appears to be the better choice, despite the cost. However, the decision is reviewed internally before finalization. As it happens, a particularly sharp risk assessor is part of the review.

The risk assessor decides to take a closer look at the meaning of the numbers. First, he discovers that the $500 \mathrm{mg} / \mathrm{kg}$ is the arithmetic mean; in fact, the soil concentrations are highly skewed, with a median of about $50 \mathrm{mg} / \mathrm{kg}$. The risk assessor finds that a lognormal distribution [with an underlying normal distribution mean of $\log _{e}(50)$ and standard deviation $\left.\log _{e}(9)\right]$ characterizes the soil concentration distribution quite well. Based on these concentrations, the risk assessor determines that about $10 \%$ of the population faces risks of $1 \times 10^{-4}$ or more, about $40 \%$ of the population faces risks of $1 \times 10^{-5}$ or more, and $75 \%$ of the population face cancer risks of $1 \times 10^{-6}$ or more.

When determining the uncertainty in the efficiency ratings of the two soil remediation methods, the analyst finds that the stated values were for soil concentrations of up to $500 \mathrm{mg} / \mathrm{kg}$. Further, Method A efficiency increases to about $95 \%$ above $1,000 \mathrm{mg} / \mathrm{kg}$, but is only about $75 \%$ efficient at small concentrations. Method B's efficiency is $95 \%$ for concentrations up to $1,000 \mathrm{mg} / \mathrm{kg}$, but then sharply decreases to $75 \%$ above $4,000 \mathrm{mg} / \mathrm{kg}$. The reduction efficiencies of the methods are shown in Figure 1.

Based on this information, the risk assessor simulates the application of both remediation methods and the resulting risks, which are shown in Figure 2. First, the point estimates of risk are $1 \times 10^{-5}$ and $5 \times 10^{-6}$ risk of getting cancer for Methods $\mathrm{A}$ and $B$ respectively. Using Method $A$, about $47 \%$ of 


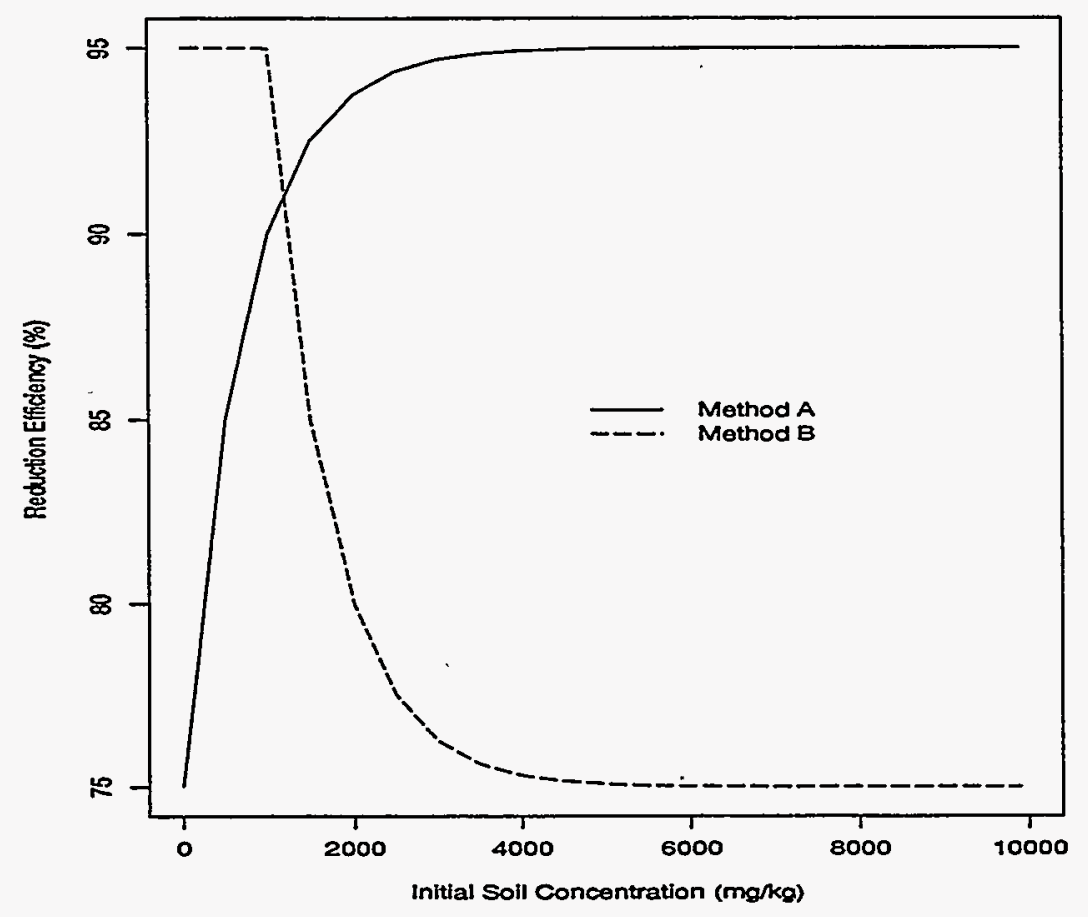

Figure 1. Plot of the reduction efficiencies of the two soil remediation methods.

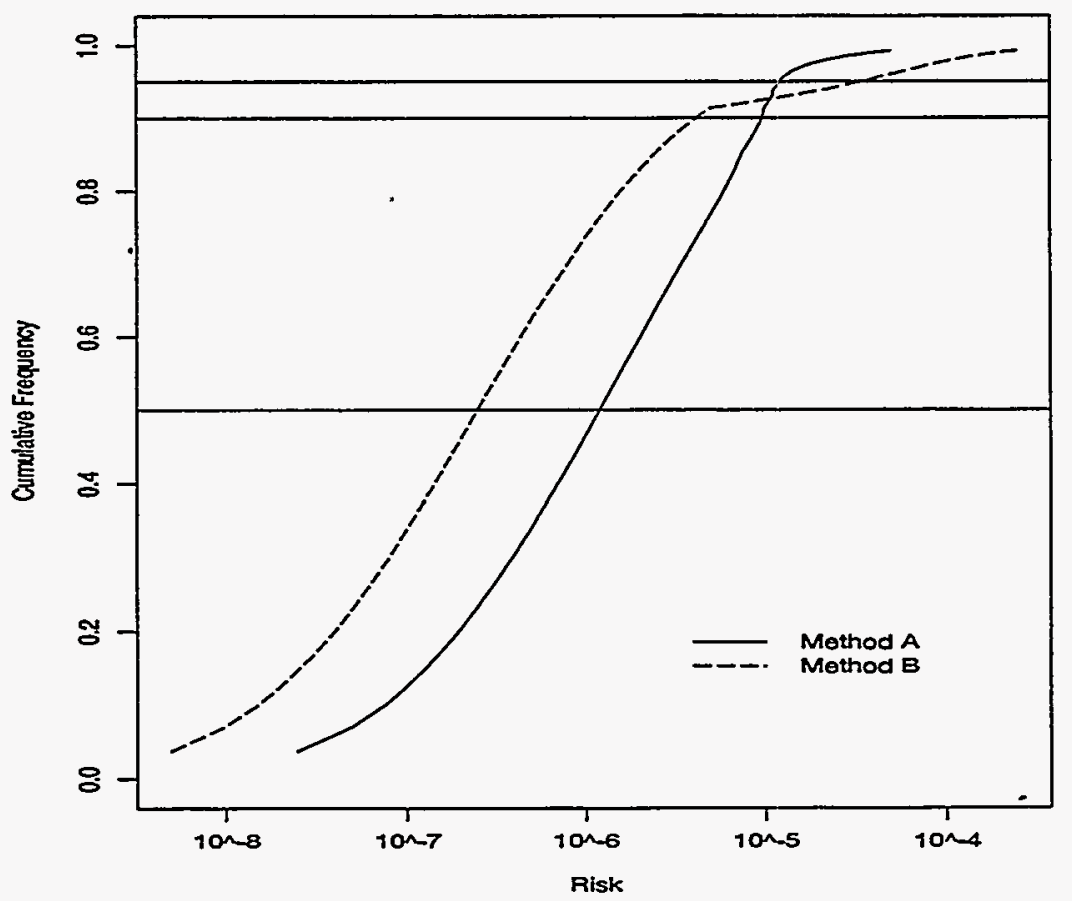

Figure 2. Plot of the simulated cancer risk distributions after application of two remediation methods. The horizontal lines are drawn at the 50th, 90th, and 95th percentiles of the distributions. 
the risk population is at less than the $1 \times 10^{-6}$ risk level, and that with Method B about 74\% of the risk population is less than the $1 \times 10^{-6}$ risk level. However, the 99th percentiles of the risk distributions are quite different. For Method A, the 99th percentile risk is about $4 \times 10^{-5}$, but for Method B it is $2 \times 10^{-4}$.

The risk analyst provides this information to the manager. The manager sees that the decision is not as straightforward as previously thought. It is not clear whether either method actually meets the requirement of reducing risk to the $1 \times 10^{-6} \mathrm{level}$. If these are the only two methods available for remediation, it is not clear which is preferred. Method A does not reduce the risk to a typical citizen (i.e., the median risk) to the $1 \times 10^{-6}$ level, $9 \%$ of the population is subject to risks greater than $1 \times 10^{-5}$, but leaves only $0.3 \%$ of the population with a risk greater than $1 \times 10^{-6}$. Method $B$, which does reduce the risk to a typical citizen to below the $1 \times 10^{-6}$ level, has $8 \%$ of the population subject to risks greater than $1 \times 10^{-5}$, but is more expensive and leaves $2.3 \%$ of the population exposed to risk levels of greater than $1 \times 10^{-4}$.

This example is more realistic than the other two in that rather than providing a clear answer for a given decision, the MCA provides a better picture of the consequences of decisions. The initial decision made by the manager was based on limited information since the uncertainty (in the soil concentrations, in particular) was not considered. When the uncertainty is included in the decision process, the decision-maker gains a better understanding of the issues that must be faced in the decision-making process.

\subsection{Possible Problems with Monte Carlo Analysis}

Clearly, a Monte Carlo analysis generally requires more effort than a BRA. The analyst must carefully balance the amount of effort against the quality of the results. To implement a "perfect" uncertainty analysis at a site would be impossible. To get site-specific information on all parameters that enter into a risk assessment would be highly unlikely. The search for the perfect risk assessment can easily lead to "analysis paralysis"-that condition in which a larger project, which the analysis is intended to support, is prevented from moving forward due to excessive introspection of the methods and data.

At some point, further data collection or more advanced modeling will lead to a case of diminishing returns. It may be difficult to determine when this occurs. This report assumes that the risks are calculated using the equations given in RAGS (EPA 1989a). Clearly, these simple models are not intended to provide particularly precise estimates of risk. Along with the large uncertainty associated with toxicity values, providing anything beyond a rudimentary distribution of the remaining input parameters may create a false confidence in the results.

Because the MCA does have more advanced data requirements, there is a concern that distinguishing a good uncertainty analysis from a bad one may be difficult (Barry 1993; Finley and Paustenbach 1994). The MCA analysis may be easily manipulated and it may be difficult to detect and judge the effects of the manipulations. This argument can also be applied to the current BRA process. Upon EPA's release of guidance for implementing MCA, concerns such as these may be alleviated.

A related problem is the lack of standardized distributions for key exposure factors (Finley and Paustenbach 1994; Finley et al. 1994). As discussed in Sections 5 and 7, standard distributions have been proposed for some parameters that have sufficient data available. Some key parameters that the risk may be extremely sensitive to are sitespecific; hence, no "standard" distribution for these parameters is likely to to be acceptable. Consequently, estimating distributions for these parameters may require a great deal of effort. In some cases, models are used to generate input parameter distributions; in these situations, the input parameter distribution is, at most, as good as the model generating it.

When input parameter values are generated from computationally complex models, the large numbers of simulations required by MCA may be 
impractical. There are techniques for improving the statistical efficiency of the general Monte Carlo method. The most well-known of these methods is Latin Hypercube Sampling (LHS), which applies a particular stratified sampling design to the input parameter distributions. Another useful method is importance sampling, in which the probability of selecting an input value is proportional to its inherent significance rather than its likelihood of occurrence; in particular, if a certain portion of the risk/exposure distribution was of great interest, input parameter values more likely to result in that output of interest could be assigned a higher probability of being sampled.

As with the BRA point estimates, there are qualitative uncertainties in the MCA results that cannot be ignored. The quality of the MCA is only as good as the selected risk model. The risk and exposure equations given in RAGS (EPA 1989a) were intended for BRA estimates; that is, these models are expected to use conservative assumptions to generate a conservative point estimate of risk. Such a model cannot be expected to produce unbiased and precise estimates of risk.

The simple BRA model may not begin to take into account the true complexities of determining exposures and risks. The model does not explicitly account for correlations between the input variables (although using an MCA or LHS sample can alleviate this problem). Age, in particular, may heavily impact risk, but is not explicitly included in the BRA risk models (there is discussion of treating children separately from adults in RAGS; others suggest treating older age groups separately as well). Care must be taken in associating the toxicity value with the appropriate exposure measure: absorbed dose, effective dose, etc. Finally, the true exposure is extremely difficult to model, due to variation in contaminant concentrations across time and space and variation in human habits.

The MCA technique does have some inherent shortcomings, but these should not pose a problem to its use in human health risk assessment. Morgan and Henrion (1990) note that under certain complex probability calculations, discrete probability tree models are preferable to MCA. In particular, if there is any Bayesian inferences to be made or the correlation structure is complex, then there may be reasons to prefer discrete probability trees. Iman and Helton (1988) point out that MCA and, in particular, LHs can induce undesirable correlations between input parameters. However, there are methods that will control these correlations.

Difficulty in estimating the tails of the input parameter distributions implies difficulty in estimating the tails of the risk distribution. It may be reasonable to compute risks for that part of the population with the greatest risk separately from the remainder of the population. This would provide for a more careful assessment of the model used and the validity of the extremes of the input parameter distributions (and the association of extreme input parameter values).

\subsection{Uncertainty, Variability, and Sensitivity}

The environmental risk assessment literature tends to make a distinction between uncertain parameters and variable parameters. The distinction helps identify which parameters to focus on to improve the uncertainty analysis. A sensitivity analysis is separate from an uncertainty analysis. However, sensitivity analyses are commonly used to help guicle the effort in the uncertainty analysis.

3.3.1 Uncertainty and Variability. In quantitative uncertainty analyses for environmental risk assessment, it is customary to distinguish between parameters that are uncertain and those that are variable. An uncertain parameter is one for which little data or information is available; hence the parameter value is literally uncertain. A variable parameter is one for which there is sufficient data or information, but the parameter does vary; for example, heights of men in a population cannot be quantified by a single height. Clearly, by these definitions some parameters may be both variable and uncertain.

Variability in a parameter can be treated in two ways:

1. Run the model on each element/member of the population or distribution. For example, a 
wind rose is a population of wind speeds and directions, and the entire wind rose can be part of a computer model of airborne transport.

2. Let the variability help generate the uncertainty distribution. For example, body weight varies among adults, and this distribution can be determined from data. A random adult ingesting vegetation or water has an uncertain body weight-that is, the postulated adult would know his/her own weight, but the analyst does not. Therefore, the distribution of adult body weights (describing variability) becomes the uncertainty distribution (describing the lack of knowledge). This method of treating variability is commonly used in environmental risk assessment.

If a variable quantity is difficult to measure, the estimated variability gives only an initial approximation of the corresponding uncertainty distribution. That is, there is uncertainty in the moments (e.g., mean, variance) of the underlying distribution for that variable quantity. There is likely to even be some uncertainty in the form of the distribution (e.g., normal, lognormal). Then this first approximation of the uncertainty distribution must be widened to reflect the fact that the population characteristics are not known very well. For example, it is known that the toxicity of a contaminant can depend upon age, sex, health, etc. That is, the toxicity is known to vary. However, typically there is little or no empirical data on toxicity in humans; that is, the toxicities are also subject to a great deal of uncertainty. The variability among humans would be treated just as variable body weights were treated above, but now the resulting uncertainty distribution must be widened to reflect the fact that even for a human of specified age, sex, health, etc., the toxicity is uncertain. In this example, this uncertainty may dominate the original variability.

When variable parameters are used in an MCA, the distribution used to characterize the variability is often considered to be an uncertainty distribution. This is argued from the point of view of sampling. When sampling from the distribution, essentially an individual from the underlying population is selected; since it is not known which individual is to be selected, it is uncertain as to which value the parameter will take on. This argument better justifies the identical handling of uncertain and variable quantities in an MCA.

Distinguishing between uncertain and variable parameters allows one to better focus efforts at improving the analysis. Essentially, a parameter with well-described variation and no uncertainty cannot be better known. That is, additional data or information will not substantially improve the quality of the uncertainty assessment. On the other hand, uncertain parameters are not sufficiently well known. For these parameters, additional data or information will potentially improve the quality of the uncertainty assessment.

From the perspective of the risk manager or decision-maker, the distinction between uncertainty and variability is not likely to affect decisions. While the final uncertainty in the risk estimates is generated as a result of uncertain and variable input parameters, the risk manager generally does not need to distinguish that part of the total uncertainty due to uncertain input parameters and variable input parameters.

\subsubsection{Sensitivity and Uncertainty Analy-} sis. Quantification of uncertainty is commonly accompanied by a sensitivity analysis. A sensitivity analysis determines how much a parameter affects the final result. For a unit change in the parameter's value, the sensitivity analysis determines how much change occurs in the final result. The uncertainty analysis quantifies the uncertainty in the final risk as a function of the uncertainties in the input parameters. Additionally, the uncertainty analysis will commonly quantify the proportion of the risk uncertainty due to each input parameter.

The sensitivity of the result may be assessed locally or globally. Local sensitivity is measured on subsets of the ranges of the input parameters. The sensitivity of the risk to local perturbations of the input parameters varies depending upon the local ranges selected. A global sensitivity analysis considers the effect of the input parameters on the risk by considering the input parameters across 
their full range of possible values. A global sensitivity analysis is related to the uncertainty analysis in that the range of the input parameters depends upon the uncertainty and variability in those parameters.

The sensitivity analysis is a useful tool for focusing the effort expended in the MCA. Prior to performing MCA calculations, the sensitivity analysis will identify which parameters' distributions to treat probabilistically. For INEL applications, this has already been done to a great extent by Smith (1994).

\subsection{Summary}

Quantitative uncertainty analysis provides numerical estimates about the quality of the results. In the case of risk assessments, the quantitative uncertainty analysis results in the range and likelihood of risks to human health or ecological receptors from exposures to contaminants. It is important to recognize that an uncertainty analysis is more than a propagation of error terms. While this report focuses on that part of an uncertainty analysis which is primarily concerned with propagation of error, the qualitative analysis, as discussed in the RAGS, is no less important. A complete uncertainty analysis should be a statement about the quality of the data, results, and/or decisions as well as the quantitative uncertainty results.

The PRA methods discussed in this document are a special case of uncertainty analyses and support the decision-making of the risk manager. Upon completion of the MCA, the risk assessor provides the results to the risk manager, who will use these results in the decision-making process. This process must also consider social, political, and financial issues, which typically are not covered under the uncertainty analysis/PRA. The focus of this report is to provide the decisionmaker with an adequate description of risk so that the decision-maker can make a more informed decision. However, the PRA/uncertainty analysis is not the only factor in making such a decision. 


\section{ISSUES}

There are a number of important issues in implementing an MCA. Resolution of these issues is not simply determining what approach is technically/ scientifically "best" (which, by the way, does not necessarily have a simple answer). As with risk assessment in general, there is no "perfect" MCA analysis of a given problem. While a perfect standard may not exist, an MCA with less uncertainty and bias and more representativeness would be considered a better analysis. Considering the current state of the DOE budget, the question needing to be answered is whether the analysis is sufficient for decision-making.

Given the lack of current regulatory guidance for Monte Carlo sampling, these issues may need to be resolved on a project-by-project basis. Most of these issues will require agreement between all the agencies (DOE, EPA, State of Idaho) with oversight of the INEL environmental programs.

Throughout the following sections, a phased approach to PRA is assumed. Each phase is a complete analysis, but is also an iterative refinement of the the previous phase. Refinements can include reducing the conservatism, using more site-specific data, using more detailed models of exposure or contaminant transport, etc. Stopping rules would include:

- It is highly probable that the overall carcinogenic risk is less than $1 \times 10^{-6}$ or the overall non-carcinogenic hazard index is less than one.

- It is highly probable that the overall carcinogenic risk is greater than $1 \times 10^{-4}$ or the overall non-carcinogenic hazard index is greater than one.

- Resources (time, budget) preclude the further refinement of the MCA. For the given level of resources required, the amount of information gained by continuing to a further refinement is not likely to significantly improve the quality of the risk assessment or decision.
- Information outside of the risk assessment provides enough useful information that, in conjunction with the risk assessment results, a decision can be agreed to by all stakeholders.

\subsection{When to Implement a Monte Carlo Analysis}

Because implementing an MCA can be timeconsuming, it is not desirable to perform an MCA with every risk assessment done at the INEL. For example, if the BRA risk is less than $1 \times 10^{-6}$ for carcinogens or the HI is less than 1 for noncarcinogens, then an MCA is not likely to affect any decision-making. Conversely, if the point estimate of risk is extremely high (e.g., greater than $1 \times 10^{-4}$ ), an MCA is not likely to significantly improve the risk manager's decision.

Within the environmental risk assessment community, there is little discussion of when to implement an MCA. Price (1993b) suggests that MCA is most useful when either the RME risks exceed the regulating criteria by less than a factor of ten, and/ or the typical estimate does not result in an unacceptable risk. Finley and Paustenbach (1994) note that as the number of exposure pathways and input parameters increase, the difference between the BRA point estimate and the 95 th percentile of the MCA risk distribution also increases. Based on Finley and Paustenbach (1994), another criteria could be whether secondary pathways (e.g., ingesting beef from cows that grazed on contaminated vegetation) are used in the risk assessment, since they found that secondary pathways can significantly increase the uncertainty in the risk estimates, resulting in an order of magnitude difference between the RME risk estimate and the 95th percentile of the MCA risk distribution.

Hattis and Burmaster (1994) provide a general discussion of when to implement an MCA analysis. Their suggestions focus on how the MCA can support project objectives. Hence, while they discuss how MCA can quantify uncertainty, they also consider how MCA is beneficial in determining whether additional information would be cost effective, how the MCA can identify and address 
vulnerable subpopulations, and how it can be used to prioritize and evaluate remediation alternatives.

A cost-benefit approach to implementing an MCA would likely be useful. Iterative refinement may also be a useful strategy. If the BRA point estimate of risk is between $1 \times 10^{-6}$ and $1 \times 10^{-4}$, a firstlevel MCA analysis might be run. This first-level MCA could be based on available data and, with great caution, expert opinion. For some future pathways, complex computer models, such as those for groundwater transport, may be required. In these cases, an iterative approach may be difficult because a significant portion of the MCA effort will be spent on running the computer model.

The MCA will be most useful when the risk manager would like the following:

- A better handle on the degree of conservatism of the BRA point estimate (i.e., determine where the point estimate falls in the resulting MCA risk distribution).

- A better understanding of the distribution of risks across the population (i.e., determine what proportion of a population is subject to a given level of risk or exposure). Also, identify those subpopulations that might be most at risk from exposure.

- More information on the ability to improve the risk estimates. Determine which pathways, contaminants, or parameters contribute most to the uncertainty in the risk or exposure. Consequently, determine whether the uncertainty can be significantly reduced by focusing effort on those causes.

- In general, then, a better understanding of the risks and the processes creating them. Stronger, more complete, risk/exposure information upon which to base decisions.

It is suggested that an MCA be implemented when the following conditions are met:

- The point estimate of risk is between $1 \times 10^{-6}$ and $1 \times 10^{-4}$ or the $\mathrm{HI}$ is between 1 and 10 .
- There are a large number of input parameters and exposure pathways subject to uncertainty.

- The cost of implementing at least a first step MCA can be justified by at least considering the cost of improving the quality of the decision versus the cost of no additional information.

\subsection{Level of Detail and Specificity}

This issue concerns the magnitude of scope of the MCA. The example shown in Section 6 is an extremely simple analysis. It uses a very simple exposure model and does not take into account any site-specific information (except contaminant concentrations in soil). The example does not use transport models. MCAs at the INEL can use this simple approach or go into much greater detail.

The decision on the level of detail required will need to be based on a cost-benefit analysis. If data is not currently available or complex transport models need to be used, then the cost for implementing a higher quality MCA may outweigh the benefits. In line with the iterative refinement approach, at some point a further refinement of the MCA results may not be worth the benefit.

Many of the risk scenarios considered at the INEL require extensive modeling transport of contaminants (e.g., to the groundwater). In some cases these models are quite complex, have many inputs, and are time consuming (costly) to run. So one issue is the extent to which the uncertainty in these outputs should be quantified. Implementing a Monte Carlo analysis of 5,000 to 10,000 runs could be prohibitive.

Under these conditions, the result of the transport model (e.g., concentration in groundwater) may be treated separately from the risk model. For example, a distribution of the concentrations in groundwater may be generated by applying LHS to the transport model. LHS is a stratified Monte Carlo sampling technique requiring far fewer runs than a regular Monte Carlo sample, while still resulting in good uncertainty estimates. This 
distribution could then be entered into a broader Monte Carlo sample for estimating the risk distribution.

Because a large proportion of the uncertainty in the risk estimates is due to the uncertainty in the toxicity values, it has been suggested that distributions for toxicity parameters could be generated by applying different high-dose to low-dose extrapolation models or using PBPK (physiologicallybased pharmacokinetic) models (Price 1993). Implementing these methods requires a great deal of time and expertise.

A good MCA will use site-specific input parameter distributions. However, site-specific data may not always be available due, for example, to cost or, in the case of future risk scenarios, unattainability. When site-specific data is not available, collection of such data may be considered. Alternatively, data from similar sites may be used in its place or regional data may provide a practical surrogate. In any case, when site-specific data is not available, caution must be applied to avoid unrepresentative exposure and risk estimates.

If surrogate data is used, the biases and uncertainties that affect the representativeness of the data should be considered (Barry 1994). That is, the ways in which the surrogate data may differ from the true site condition should be assessed. If biases can be identified and can impact the results, then they should be corrected for. If biases cannot be identified, then a method for quantifying the uncertainty introduced by the surrogate data should be implemented.

If further refinement is needed but the costs of implementation are prohibitive, other methods could be explored. Alternative exposure and toxicity models may be worth considering. Switchover analysis with the current data could be insightful. A switchover analysis determines where in an input parameter's distribution, the (discrete) decision would change. So, for example, one might determine what contaminant concentration results in a probable risk of $1 \times 10^{-6}$, and compare this value to the current site concentrations to determine if it is feasible to clean the soil down to that concentration.

\subsection{Selection of Parameter Distributions}

Site specificity is one aspect of the larger subject of selection of parameter distributions. The issue of development of parameter distributions is critical to a meaningful MCA. For some parameters, there may be plenty of observed data to arrive at a highly accurate distribution/characterization; some other parameters' distributions may be specified from theory. However, there will always be parameters for which there is not enough available data nor understanding to get an accurate distribution.

A number of references discuss distribution selection (in particular, Morgan and Henrion 1990; and Finley et al. 1994). Region III of the EPA expects that the PDFs will be based on peerreviewed literature and site-specific data (EPA 1994). Regardless of the source or method of selection, the selected distributions must be agreed to by all stakeholders.

"Standard" PDFs for risk assessment parameters are becoming more available in the literature. Finley and Paustenbach (1994) cite 11 exposure parameter studies which they believe have sufficient data and applicability for which standard distributions can be developed. Brorby and Finley (1993) present some suggested standard PDFs. Finley et al. (1994) present standard distributions for 10 exposure parameters and provide valuable advice on selecting distributions. Table 1 identifies the parameters considered in these studies.

Distribution selection is not as simple as finding a reference in the literature. For example, consider soil ingestion by children. EPA (1989b) cites at least 7 studies of soil ingestion by children, with average amounts ranging from 20 to $100 \mathrm{mg} /$ day (not including pica behavior). Of course, some of these studies may have flaws which would eliminate them from consideration (e.g., not adjusting for trace elements in food). Still 3 or 4 sets of reasonable data may remain. Now should the "best" data be selected or should the results be collapsed 
Table 1. Risk equation parameters for which enough data are available to develop standard probability density functions (from Finley and Paustenbach 1994).

\begin{tabular}{ll}
\hline Soil ingestion rates (children) & Total water ingestion rates \\
Body weights & Total tapwater ingestion rate \\
Vegetable ingestion rates & Total skin surface area \\
Particle deposition rates & Skin surface area by body part \\
Dermal soil adherence & Time in a shower \\
Mother's milk ingestion rates & Total fish consumption \\
Inhalation rates & - Marine \\
Exposure duration & - Freshwater \\
- Time since last job change & - Shellfish \\
- Residential occupancy period & - Recreationally harvested fish \\
\hline
\end{tabular}

(meta-analysis)? For this example, Thompson and Burmaster (1991) selected the "best" data set to determine a PDF. They used the data set from the original study to calculate the PDF. Brorby and Finley (1993) also selected the study they considered "best" for this parameter-but it was a different study than that selected by Thompson and Burmaster.(1991). In fact, Brorby and Finley (1993) consider the data used by Thompson and Burmaster (1991) to be of low quality (Thompson and Burmaster 1991 made some transformations of the data to account for the data's shortcomings). Hence, it is necessary to thoroughly review the available data on a parameter and evaluate its quality. It may be necessary to enlist the expertise of relevant technical professionals to help determine which data sets are of sufficient representativeness and quality.

In the EPA Region III guidance (1994), it is recommended that PDFs be developed from professional judgment only when no data is available and only in the form of triangular or uniform distributions. Conversely, Seiler and Alvarez (1994) present a strong argument against using triangular or uniform distributions when little or no data is available, as discussed in Section 5.2.2. Regardless of the distribution used to represent the parameter, the selected distribution must be technically defensible.
With respect to the MCA protocol, then, there are a number of issues to be considered. Specifically, while the MCA protocol can provide guidelines on selecting and developing PDFs for parameters, recommending distributions (beyond what is already available in the literature) is a task unto itself and requires knowledge in the area of the parameter under study.

In any case, all agencies and stakeholders involved in the risk assessment and decisionmaking process must agree to the selected distributions. This should help guarantee that the distributions are sufficiently broad to account for all uncertainties in the parameters.

Development of a distribution for the toxicity parameter merits particular attention. The potency parameter used in risk assessment is arguably the most critical parameter in the risk model. It is the sole link between the exposure assessment and the risk. The toxicity/potency of a chemical is typically determined from dose-response studies, typically animal studies.

The problem with the potency measures is the high degree of uncertainty in them. While there are a number of reasons for this uncertainty, the two largest sources of uncertainty are likely (1) extrapolation from animal studies to humans, and (2) extrapolation from high doses to low doses. 
For carcinogens, the potency is given by the "slope factor" (SF, also called a "cancer potency factor") and has units of (kg.d)/mg. The SF is the risk per dose. It is calculated as the upper $95 \%$ confidence limit of the slope of the (log) linear relationship between dose and response (usually frequency of tumors), with intercept at zero dose (i.e., no threshold). Clearly, the no-threshold assumption is at least conservative (if not realistic); however, the low-dose extrapolation model is more difficult to characterize with respect to conservativeness.

For non-carcinogens, the potency is given by the "reference dose" (RfD) and has units of $\mathrm{mg} /(\mathrm{kg} \cdot \mathrm{d})$. Non-carcinogens are assumed to have a threshold, that is, a dose below which there is no adverse affect. The RfD is essentially the threshold (the "No Observed Adverse Effect Level" or NOAEL) from chronic exposure studies. More specifically, the $\mathrm{RfD}$ is the NOAEL divided by uncertainty factors.

There is evidence supporting extrapolation of potency results from animal studies to humans (for example, see Allen et al. 1988). However, there is a concern that correlations in potency between different species may be an artifact of the experimental design (see Freedman et al. 1993). There are also known instances of chemicals that are carcinogenic in humans but apparently not in animals (for example, benzene and arsenic). PBPK modeling was designed to overcome the difficulty of extrapolating animal study results to humans. It has been applied in monte carlo risk assessments (e.g., Cronin et al. 1995).

These uncertainties propagate into the final risk estimate. Price (1993a) states that "compounds ... for which low-dose human potency is based solely on extrapolation from high-dose animal experiments, uncertainties in risk are dominated by the uncertainty in potency." He further cites a 1985 Office of Science and Technology report which states that the "estimated carcinogenic potency does not necessarily give a realistic prediction of the risk. The true estimate of risk is unknown, and may be as low as zero." Finley and Paustenbach (1994) state that "it is likely that the inherent conservatism and degree of uncertainty in the slope factors far outweigh the lack of precision in the exposure assessment."

Developing distributions for potency can be extremely difficult and controversial. Finley and Paustenbach (1994) note that SF PDFs for 2,3,7,8-TCDD, benzene, and benzo(a)pyrene have been proposed. While they propose generating PDFs by applying a variety of low-dose extrapolation models to the best available data, this likely will not quantify all the uncertainties in these parameters. Clearly there is a need to better characterize the uncertainty distributions of contaminant toxicities. Again, when such distributions are developed they will need to be agreed upon by all agencies and stakeholders involved in the decision-making process.

\subsection{Sensitivity Results}

Most of the literature on MCA (e.g., Thompson, et al. 1992; Finley and Paustenbach 1994; Burmaster and Anderson 1993) suggests that for each MCA an initial sensitivity analysis should be implemented to determine which parameters should be varied and which can safely be held constant without significantly affecting the final risk distribution. The authors agree that in most INEL MCA applications, an initial sensitivity analysis should be implemented. However, when similar scenarios, models, and contaminants are addressed in other previous INEL MCAs, it may be possible to rely on the previous sensitivity analysis results.

The sensitivity results will allow the risk assessor to better determine which parameters should be treated probabilistically. Burmaster and Anderson (1993) suggest the parameter be treated probabilistically if the range of the parameter accounts for a dominant fraction of the variation or range in the predicted risks.

The literature is somewhat vague on how to implement the preliminary sensitivity analysis. While some suggest developing PDFs or CDFs for all parameters of interest and then running the sensitivity analysis to focus on the most important parameters, this would be redundant. Another 
method determines rough upper and lower limits on the parameter and examines how much the risk changes over this range of the parameter (while holding the remaining parameters constant). This method, the nominal range method, is discussed in detail in Section 5.

At the INEL, Smith (1994) used different criteria to identify parameters that can be expected to require a more focused effort for developing distributions. These parameters are identified in Table 2 and are expected to be highly uncertain and the risk is highly sensitive to changes in these parameters. Although the risk results may be most dependent upon the assumptions/distributions developed for these parameters, they are not necessarily the only parameters that should be treated probabilistically in a particular MCA project.

\subsection{Correlation Between Parameter Uncertainty Distributions}

One limitation of current MCAs is characterizing and accounting for correlations between input parameters. While there are methods for handling correlations in MCA (Morgan and Henrion 1990), there is little data available on the strength of correlations between parameters commonly used in risk and exposure assessments.

Evidence suggests that it is important to account for correlations between parameters; the degree of importance depending upon the strength of the correlation. Smith et al. (1992) found that ignoring correlation may seriously bias the resulting risks; however, they also conclude that in some circumstances correlation can be ignored, and give a method for assessing how much effect correlation may have on results. Circumstances in which correlations may be ignored (because they will not substantially impact results) include:

\section{- Weak correlations}

- Correlations between variables with low influence/sensitivity.

Burmaster and Anderson (1994) consider a correlation greater than 0.6 (in absolute value) to be "strong." While this use of a cutoff value is an oversimplification, Burmaster and Anderson (1994) suggest performing some simulations with and without the correlations to see how much difference they can make.

With the possible exception of treating children separately, age is not generally considered in the EPA exposure models. However, age does potentially account for a large proportion of the variation in some of the input parameters, such as weight and toxicity. Finley et al. (1994) suggest that a way to minimize errors due to correlation between parameters that are a function of age is to use agespecific distribution functions, breaking the exposure assessment calculations down by age.

When two (or more) parameters are positively correlated, and are summed in the model, then the mean will be unaffected but the variance will

Table 2. Sensitive and uncertain parameters in INEL risk assessments (Smith 1994).

$\begin{array}{ll}\text { Contaminant leach rate from } & \begin{array}{l}\text { Contaminant leach rate from } \\ \text { sludges }\end{array}\end{array}$

Diffusion rate of volatiles and semi-volatiles out of waste form into gas phase

Reference doses (nonradiological)

Sorption coefficients for interbed sediments
Hydrological properties of basalt fractures

Slope factors (nonradiological)

Thicknesses of individual sedimentary interbeds
Contaminant leach rate from surface washoff

Hydrological properties of interbed sediments

Sorption coefficients for disturbed areas (source zone) 
increase (than if the correlation is not accounted for). If the positively correlated parameters are multiplied, then both the mean and variance will be larger than if the correlation is not accounted for; if they are divided, then the mean and variance will be smaller than if the correlation is not accounted for.

When using complex computer models (e.g., contaminant transport models) the correlations may be accounted for to some extent by the models. Complex causation mechanisms that cannot be accounted for by simply including a correlation coefficient must be part of the model, if the MCA results are to be representative. As a relatively simple example, the radionuclide activity in a waste repository will change over time due to decay. The amount it changes is a function of the half-lives of the radionuclides. However, the relationship between activity and half-life cannot be quantified by the correlation coefficient; rather, the model must take decay into account.

\subsection{Expert Opinion}

When dealing with uncertain parameters (as opposed to variable parameters), a degree of subjectivity is required when developing the distribution. Eliciting expert opinion is one way to obtain distributions for uncertain parameters.

When data are not available and bounding distributions (e.g., uniform, triangular) do not provide enough specificity, then subjective probability distributions may be developed. The subjective methods can roughly be divided between informal and formal methods. Informal methods include self assessment, brainstorming, causal elicitation (without structured efforts to control biases), and taped group discussions (Barry 1994). While the informal approaches are clearly less costly in the short term, they may provide an incomplete description of the uncertainty in the parameter and the results will be more difficult to defend. Formal methods are likely to perform significantly better than informal methods under the following conditions (Barry 1994):

- Data are unobtainable and existing data must be supplemented
- Information to be gathered will have a major impact on the results of the study

- The available information is highly variable

- The issues are complex

- The representativeness of available data is questioned

- Existing findings must be supplemented, interpreted, or extended because of new data

- Several experts will be employed individually or as a team

- The findings are expected to undergo close scrutiny or subject to legal challenge

- Expert opinions will be used extensively by other studies

- The costs of redoing an informal study that failed to withstand criticism or that used faulty methods are expected to exceed the costs of conducting a formal elicitation study.

Use of expert judgment has weaknesses, one of which is a substantive problem in quantifying the tails of the distribution. The tails of the resulting risk distribution are of greatest regulatory interest and are most influenced by the tails of the input parameter distributions. Expert opinion is least effective at estimating tails of the distribution due to the greater uncertainty associated with extreme, rare events. Morgan and Henrion (1990) provides an overview of three methods for formal elicitation of expert opinion, along with further references.

\subsection{Bounding the Assessment/Problem}

The general form of the question/decision to be considered by risk analysis is "Does this site present a significant risk to humans/environment?" The answer to this question may depend upon how it is framed. When determining significant risk, the assessor must understand what population is to be considered. In the past, risk assessments have tended to focus on the most sensitive subpopulations 
(that is, they have been conservative). The EPA (1992a) suggests three portions of the population should be considered: the TUBE, a member of the population in the top $10 \%$ of risk, and a median population risk.

Some authors have cited a need to consider tails of distributions separate from the remainder of the distributions (e.g., Barry 1994; Lambert et al. 1994; Haimes et al. 1994). Whether this possibility should be developed and described in this document depends upon the framing of the assessment question. There is consensus that standard methods for parameter distribution selection do not adequately address the tails of the distributions. Hence, if the assessment question is framed such that the tails of distributions are important, then discussion of tail estimation should be included. However, if it is decided that, for example, the TUBE be a point estimate (as it should be) and the median and 90 th percentiles will be the driving risk values, then the tails may likely be ignored in most cases.

As with many of the other issues discussed here, the scenarios, pathways, models, endpoints, etc. must be agreed upon by the stakeholders. In order to meet the expectations/requirements of all the stakeholders, the problem must be clearly defined and understood by all stakeholders.

\subsection{Ecological Risk Assessment}

The use of probabilistic exposure models for ecological risk assessments at contaminated sites at the INEL has the same advantages and disadvantages as the human health efforts. The development of distributions, however, may be even more difficult due to lack of available information on the multiple receptors at the site. The complex food web and multiple species issues may also be problematic. 


\section{METHODOLOGY}

In this section, the methodology for performing an MCA is developed. There are three critical steps in the process.

1. Implement a sensitivity analysis of the risk, identifying parameters that most impact the risk estimate.

2. Determine appropriate probability density functions (PDFs) or cumulative distribution functions (CDFs) for parameters identified in Step 1.

3. Implement Monte Carlo sampling of input distributions to calculate the risk distribution.

- Determine the number of Monte Carlo samples needed to get a reasonably precise estimate of the risk distribution.

- Assess the reproducibility and representativeness of the results. Also, implement a final sensitivity analysis showing which parameters have the largest impact on the overall uncertainty in the risk estimates.

4. Present a graphical summary of the results.

The following sections provide more details on the implementation of these steps.

This document recommends the use of Monte Carlo simulation for risk distributions. Monte Carlo methods, including LHS, are conceptually and computationally simple to implement. Environmental risk assessment commonly uses complex transport models. Most other methods of uncertainty analysis cannot handle computational complexity as easily as Monte Carlo methods. However, other methods of uncertainty analysis are available. So, while most, if not all, the environmental risk analyses at the INEL are well suited to MCA, other methods might be considered. Alternative techniques are introduced and further referenced in Morgan and Henrion (1990).

\subsection{Initial Sensitivity Analysis}

Once the decision has been made to proceed with MCA, the initial step is to determine which parameters most impact the risk estimates. When the range of a parameter's uncertainty distribution account for a dominant fraction of the range in the predicted uncertainty in the risks, the risk would be considered sensitive to that parameter (Burmaster and Anderson 1993).

To determine which parameters meet this criteria, an initial sensitivity analysis should be implemented. The method suggested is called the "nominal range" sensitivity analysis (Morgan and Henrion 1990). Before implementing the nominal range method, the user may find it useful to initially decide (subjectively) which parameters most impact the risk. These initial identifications, though subjective, may be useful later in assessing the representativeness of the results from the nominal range analysis.

5.1.1 Nominal Range Method. Essentially, this method measures the change in risk over the expected range of parameter values. Those parameters that cause the greatest change in risk are considered the most important and should be handled probabilistically in the MCA. The nominal range method identifies those parameters which account for a dominant fraction of the range in predicted risks. Except for some problems discussed later, the method is straightforward to implement.

1. Estimate a reasonable minimum, median or average, and maximum for each parameter. These values are expected to be rough estimates, and should not require a great effort to determine. Expending too much effort would defeat the purpose of the initial sensitivity analysis: to focus the effort on the most important variables.

2. For each parameter, calculate the risk at the minimum and maximum values while holding all the remaining parameters at their median or average. The "swing weight" for the parameter is then the absolute value of the 
difference between the risks at the maximum and minimum for that parameter.

3. Rank the swing weights from largest to smallest; the largest swing weights represent the most important parameters. A rule of thumb for the cutoff has not yet been established. Consequently, a visual inspection of the rankings combined with professional judgment is required.

\subsubsection{Some Caution Is Required. Certain sit-} uations may cause serious problems with implementation of the nominal range method. First, there may be so many parameters or the model may be so complex that it is impractical/prohibitive to vary each parameter. Second, the presence of interaction between parameters may invalidate the results.

When there are many parameters, it may not be reasonable to vary each one, especially when the risk calculations are expensive to run. This is particularly a problem when running transport model codes for exposure estimates. In these situations, the analyst could use prior knowledge about which parameters are most important in the code, and vary only those parameters. Alternatively, it may be possible to use of fractional factorial designs to reduce the number of runs required to determine the most sensitive parameters.

The presence of interaction between parameters can lead to invalid results. Interaction occurs when the effect of one parameter on the risk depends upon the level of another parameter. For example, because a child's blood/brain barrier is not fully developed, it is more susceptible to lead poisoning than an adult's. A low concentration of lead which may not have any significant impact on an adult may have devastating effects on a child. Hence, the effect of lead on health depends upon age. This is an interaction.

Interaction can be assessed through a more rigorous selection of parameter levels than those produced using the nominal range method. If interaction is suspected, the analyst should consider methods that can assess interaction, such as factorial designs.

\subsection{Distribution Selection}

As discussed previously, selecting parameter distributions for use in the analysis is a critical and often difficult step in MCA. Selection of representative distributions is essential to obtain meaningful results from the MCA. Care must be taken in selecting accurate descriptions of the range and likelihood of parameter values so that the distributions adequately describe both the variation and uncertainty in the parameter. Distribution selection can be difficult due to a lack of available data, amount of effort required, and technical complexity.

The following Sections, 5.2.1 through 5.2.3, give more details on parameter distributions and their selection. The first section discusses the types of quantities that may enter a risk equation and how they should be handled in an MCA. The second section gives an overview of the types of distributions that are commonly used to characterize a parameter. The third section discusses methods for selecting distributions.

5.2.1 Types of Quantities. Morgan and Henrion (1990) identify six types of quantities that may enter a risk or exposure model as shown in Table 3. A. review of the MCA literature might imply that distributions can and should be developed for all parameters in the risk assessment equations. Morgan and Henrion (1990) present a strong argument against excessive use of probability distributions. Their main thesis is that certain quantities in a risk equation are not adequately characterized by probability distributions and entering them as such can result in misleading or vague results.

While distributions may be developed for any quantity in a risk or exposure model, it is important to recognize the limitations and implications. As shown in Table 3, Morgan and Henrion (1990) recommend that only empirical parameters be treated probabilistically in an MCA. Uncertainty in other 
Table 3. Summary of types of quantities used in risk/exposure equations (Morgan and Henrion 1990).

\begin{tabular}{lll}
\hline \multicolumn{1}{c}{ Type of quantity } & \multicolumn{1}{c}{ Example } & \multicolumn{1}{c}{ Treatment of uncertainty } \\
\hline Empirical parameter & $\begin{array}{l}\text { Contaminant concentrations, body } \\
\text { weight, soil ingestion rate } \\
\text { Defined constant }\end{array}$ & $\begin{array}{l}\text { Probabilistic, parametric, or } \\
\text { switchover }\end{array}$ \\
Decision variable & Remediation method & Certain by definition \\
Value parameter & Risk tolerance, value of life & Parametric or switchover \\
Index variable & Time, location & Parametric or switchover \\
Model domain parameter & Time increment & Certain by definition \\
\hline
\end{tabular}

quantities should be treated through parametric analysis or through switchover analysis.

In parametric analysis, the uncertain parameter is varied over a range of possible values without specifying the probability of that value occurring. That is, the model is analyzed for a range of values of the uncertain parameter. This allows one to assess whether changes in the parameter's values has any effect on the response.

In switchover analysis, the uncertain parameter's values are back-calculated to determine where in the range of the parameter's values a change in decision would occur (if anywhere). This results in a series of possible decisions and the uncertain parameter's values that are compatible with that decision. For example, the method used to remediate a site will affect future risk. For each remediation method considered, one could back-calculate to determine under which conditions (of the remaining parameters) the risk is minimized. This would be a switchover analysis.

Before discussing the types of quantities encountered in risk and exposure assessment, it should be noted that the determination of the type of a given quantity may vary depending upon the situation. For example, when determining Maximum Concentration Limits (MCLs) for drinking water, the EPA may consider the MCL to be a decision variable. However, the INEL, when assessing compliance with regulatory standards, would consider the MCL a defined constant.

5.2.1.1 Empirical Parameters. Empirical parameters or chance variables are quantities that, at least in theory, are measurable properties of the system/population being.modeled. Many variables in exposure and risk models are empirical quantities. Examples include carcinogenic slope factors, daily consumption of water by a 1- to 5-year-old child, and number of days a person is exposed to a contaminant. The key property of an empirical parameter is that it represents a measurable property of the population/system.

There are generally five types of empirical parameters used in human health risk assessment that may require distributions for use in an $\mathrm{MCA}$ :

1. Contaminant concentrations

2. Slope factors and hazard indexes

3. Certain pathway parameters

4. Transfer coefficients

5. Demographic statistics.

Empirical parameters are the only type of quantity that Morgan and Henrion (1990) recommend to be treated probabilistically (i.e., through probability distributions) in an MCA. Unlike other types of quantities that may be entered into an MCA, 
empirical parameters do have true, observable values; however, these true values are typically unknown and hence subject to both uncertainty and variability.

This guidance recommends that empirical parameters should be the only parameters treated probabilistically. The remaining types of quantities are presented for informational and awareness purposes.

5.2.1.2 Defined Constants. Defined constants are physical constants, including values such as $\pi$, Planck's constant, and the number of days in the year. Obviously, these values are constant by definition and would not be varied in an MCA.

5.2.1.3 Decision Variable. Decision variables are parameters over which there is direct control. For example, the remediation method used to clean up a site would be a decision variable since there is a choice of methods available that could be implemented. Decision variables have no "true" value, but have a set of possible values, one of which must be selected. Decision variables are not "uncertain" and cannot be treated probabilistically. They are commonly analyzed through switchover techniques to help the decision-maker determine the optimal value of the variable.

5.2.1.4 Value Parameters. Value parameters represent aspects of the preferences of the decision-makers or the people they represent. Two common examples include the controversial "value of life" and the risk tolerance (the degree of risk aversion). These parameters are often treated probabilistically, but Morgan and Henrion (1990) argue against this treatment for two reasons:

1. "Value parameters tend to be those that have the greatest uncertainty and may contribute most to the decision. Probabilistic treatment may hide the impact of this uncertainty so the decision-maker may not be able to see the implications of their possible alternative value choices."
2. "It is often an important purpose of analysis to help people to choose or clarify their values. If the analysis reveals that the value parameter does not affect the decision, it can be safely ignored. Alternatively, if the decision is found to be dependent upon the value parameter, then careful thought and refinement will be worthwhile."

Morgan and Henrion (1990) further argue that value parameters are best treated parametrically (i.e., each possible realization of the value parameter is analyzed separately). By so doing, the researcher can gain insight into the implications of possible alternative value choices.

5.2.1.5 Index Variables. Index variables are used to identify a location or cell in the spatial or temporal domain of a model. Examples of index variables include year in a multi-year model and grid location in a spatial model. Index variables may also identify a member of a set; for example, the RWMC is an element of the set of (potentially) contaminated sites at the INEL. As with defined constants, index variables have no uncertainty associated with them.

5.2.1.6 Model Domain Parameters. Model domain parameters bound the spatial and temporal extent of the system being modeled. They also specify the spatial and temporal increments of the index variables. They are important in both a PRA and BRA analysis. For example, for a future risk scenario, the last year modeled specifies the temporal domain of the model; for contaminant transport, depth to groundwater might be considered a domain parameter. Domain parameters also specify the step size of index variables. Domain parameters may also define baseline properties, such as background concentrations of metals in soils.

Because the model domain parameters control the precision of the representation and the computational complexity of the model, model domain parameters can substantially impact the uncertainty analysis. The finite domain of analysis and finite degree of detail gives rise to "approximation uncertainty." It is worthwhile to vary model domain parameters parametrically to examine how they affect the results of the analysis. 
Awareness and understanding of how the model domain parameters affect risk estimates is critical. This aspect of models is too easily overlooked. In particular, the temporal domain should be well understood when applying PRA techniques.

The magnitude of the time-scale may significantly impact a parameter's distribution. That is, short-term variation often differs from long-term variation. Examples in which short-term variation is actually larger than long-term variation are fairly common; indeed, when averaging over periods of time to get daily values, the variation will decrease as the averaging time increases.

Uptake parameters, whether ingestion or inhalation rates, for infrequently encountered substances can pose a particular problem in defining model domain parameters. Finley et al. (1994) specifically cite consumption of shellfish and freshwater fish. A short-term survey will conclude that a vast majority of the population do not eat either of these since they are infrequently eaten by individual population members. Hence, basing an uptake distribution for chronic uptake on such results will result in a poor characterization if the temporal domain is much longer than the survey's temporal domain.

5.2.2 Types of Distributions. This guidance recommends that empirical variables are the only parameters to be treated probabilistically in an MCA. These variables may be entered into the MCA in the form of a probability distribution. In this section, the forms that the distribution may take are examined. This is essentially an introduction to some commonly used distributions. For more information, the reader should refer to more definitive texts such as Evans, Hastings, and Peacock (1992) or Johnson and Kotz (1969, 1970a, 1970b, 1972).

The probability distribution must identify the values that the variable can achieve and the likelihood that the value(s) will occur. This can be done using cumulative distribution functions (CDFs) which show the probability that the variable will take on a value less than or equal to the specified value. When the $C D F$ is continuous, the derivative of the $\mathrm{CDF}$ is the probability density function (PDF), often simply called the density. For a discrete distribution, the CDF is a step function, and the difference of successive values is the probability mass function (PMF).

While the shape of the distributions of the risk equation input parameters generally have little or no effect on the resulting median risk values, they can have a large effect on the tails of the resulting risk distribution. Since the tails of the risk distribution are always the most uncertain and often of most interest, careful selection of input parameter distributions that adequately characterize the extreme values of the parameter is important.

A case in point is the common use of the uniform distribution when there is little or no available information on a parameter. It is not clear how this trend started; possibly because the distribution has such a simple form it was convenient to use; also, by assigning each value an equal probability of occurrence (within the range of the parameter), it is considered a reasonable descriptor of a high degree of uncertainty.

Seiler and Alvarez (1994) argue that use of the uniform distribution requires far more stringent knowledge requirements than the use of other distributions. The uniform distribution is a twoparameter distribution; the parameters represent the minimum and maximum values. According to Seiler and Alvarez (1994), knowing exactly what these limits are requires a tremendous amount of knowledge, further saying that points even near the extreme values of the range have equal probability of occurrence also requires a great deal of knowledge since it goes against common sense (for many risk input parameters).

On the other hand, there is some support for using simple distributions like the uniform and triangular. Use of the uniform distribution is somewhat conservative if the minimum and maximum are appropriately selected. Also, EPA Region III (EPA 1994) requires the use of uniform distributions when distributions are based on expert judgment. Morgan and Henrion (1990) argue that the use of these distributions would indicate that the 
true distributions are not precisely known and may help prevent over-interpretation of the results.

Characterization of the tails of a parameter distribution can be a particular problem. Since risk managers are commonly interested in the tails of the risk distribution, and these tails are usually a result of combinations of extreme values of the input parameter distribution, tail characterization may be of particular importance.

If the INEL protocol is to follow current EPA (1992a) exposure assessment guidance, then the focus will be on the TUBE, 90th percentile or higher, and the median. The TUBE is derived theoretically and will not rely on MCA. The 90th percentile and median can be estimated from MCA results (indeed, nonparametric confidence limits on these values can be developed). Finley et al. (1994) indicate that in their experience, estimation of the median and 90-95th percentile are relatively unaffected by extreme values. Hence, it may be that no special consideration of tails and extreme values is warranted.

If values beyond the 95th percentile of the risk or exposure distribution are of interest for the decision-making process, then special methods for handling tail distributions may be required. Lambert et al. (1994) present a good introduction to extreme value statistics and methods for handling tails of distributions. Basically, they argue that the assessment of the tail of the distribution should often be performed separately from the assessment for central values.

Distributions can be used to describe either a set of observable values or the likelihood that an unknown but estimable quantity takes on a particle value. In the first case, the distribution describes the probability or frequency with which the possible values occur in the population. Distributionsobservable data can be either discrete or continuous. For example, the lognormal distribution provides a good description of adult body weights (Brorby and Finley 1994). In the latter case, the distribution represents variability and uncertainty in a parameter. Distributions of empirical parameters will be continuous. For example, the expected proportion of the contaminant in food that is absorbed would likely be described by a beta distribution.

The risk analyst may have observed data; in which case, the analyst will try to fit the observed data to a known distribution. The parameter(s) of that distribution have an uncertainty distribution, and perhaps a variability distribution. The form of the distribution of a parameter may be determined from statistical estimation procedures (e.g., binomial data often lead to a beta distribution for $p$ ), or may be based on expert judgment. In any case, the uncertainty or variability distribution may have one of the forms given next.

Plots of the most commonly used PDFs and PMFs are shown in Figure 3. The characterization and applicability of these distributions are briefly discussed in the following sections. The first five distributions, the normal, lognormal, exponential, Weibull, and gamma, can apply to both data and parameters. The next three distributions, the beta, uniform, and triangular, are typically used in describing parameters. The final distributions, the binomial and Poisson, are typically used in describing data.

5.2.2.1 Normal Distribution. The normal, or Gaussian, distribution is the familiar bellshaped distribution. It is a commonly used distribution for describing unbiased measurement error and sums of random variables. This and the lognormal are the most familiar distributions and may therefore be somewhat overused.

The PDF for a normally distributed variable is given by

$f(x)=\frac{1}{\sqrt{2 \pi \sigma^{2}}} \exp \left(-\frac{(x-\mu)^{2}}{2 \sigma^{2}}\right) ;-\infty \leq x \leq \infty$.

The mean and variance are given by $\mu$ and $\sigma^{2}$, respectively. There is no closed form of the CDF, though tables and numerical methods are available. Many of the following distributions converge to the normal distribution as the coefficient of variation, $v$, gets small, where $v$ is the standard deviation divided by the mean. 
a. Normal

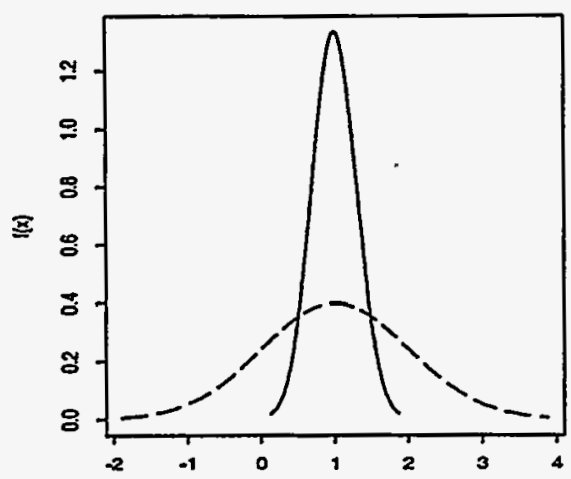

d. Weibull

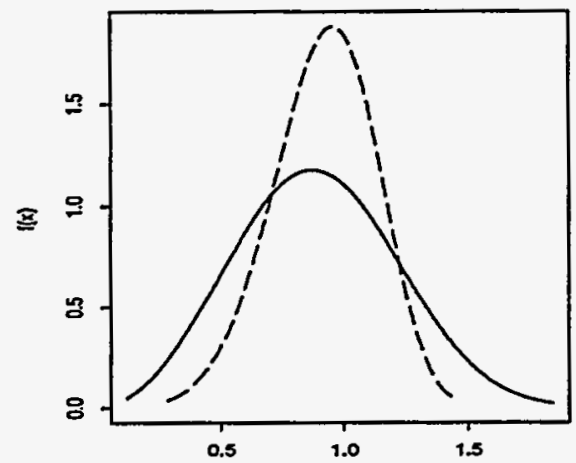

g. Triangular

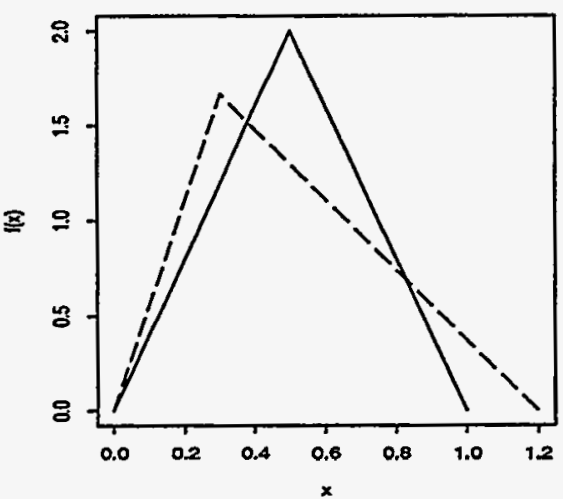

b. Lognormal

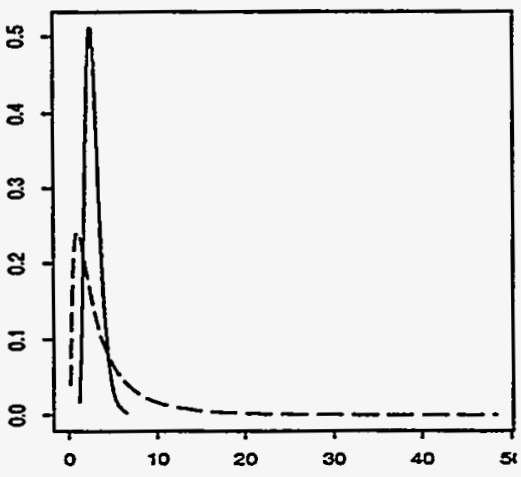

e. Gamma

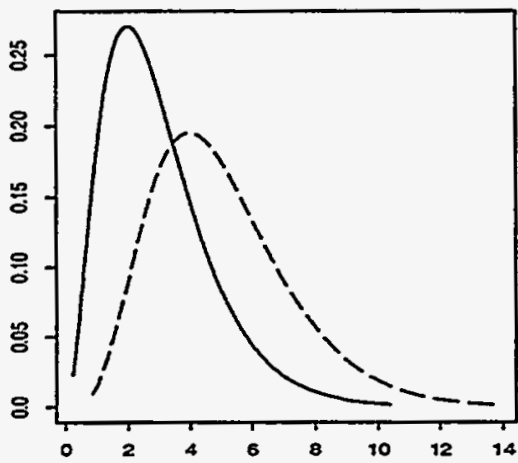

h. Poisson

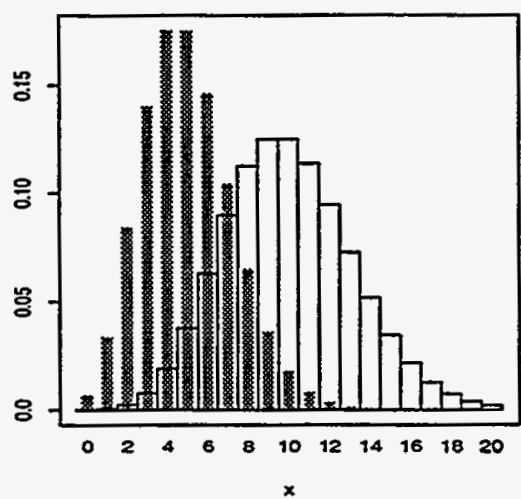

c. Exponential

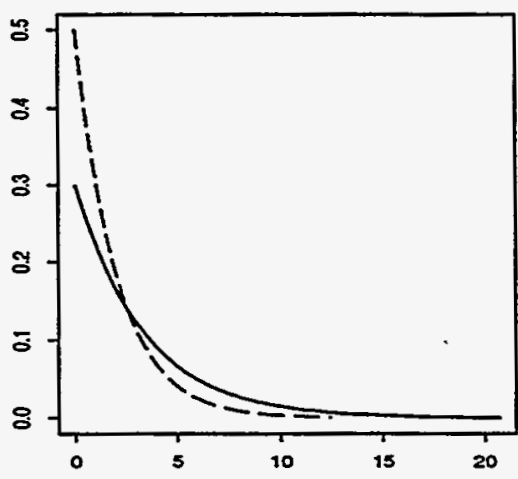

f. Beta

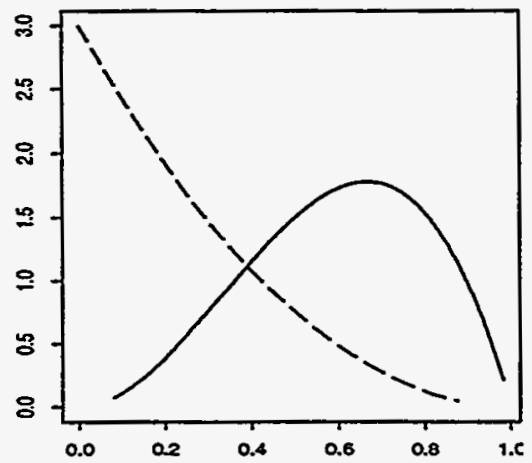

i. Binomial

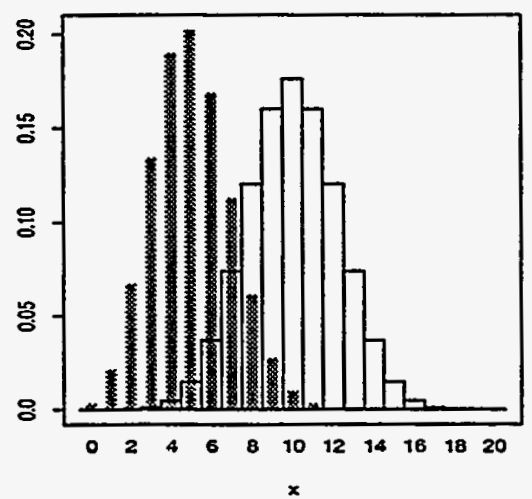

Figure 3. Plots of the density or mass functions for common distribution types. Parameter values are as follows (see Section 5.2 for parameter definitions): a. Normal, solid: $\mu=1, \sigma=0.3$; dashed: $\mu=1, \sigma=1$. b. Lognormal, solid: $\mu_{\mathrm{y}}=1, \sigma_{\mathrm{y}}=0.3$; dashed: $\mu_{\mathrm{y}}=1, \sigma_{\mathrm{y}}=1$. c. Exponential, solid: $\lambda=0.3$; dashed: $\lambda=0.5$. d. Weibull, solid: $\gamma=0, \alpha=3, \beta=1$; dashed: $\gamma=0, \alpha=5, \beta=1$. e. Gamma, solid: $\gamma=0, \alpha=3, \beta=1$; dashed: $\gamma=0, \alpha=5, \beta=1$. f. Beta, solid: $\alpha=3, \beta=2, \gamma=0, \theta=1$; dashed: $\alpha=1, \beta=3, \gamma=0, \theta=1$. g. Triangular, solid: $\alpha=0, \gamma=0.5, \beta=1.0$; dashed: $\alpha=0, \gamma=0.3, \beta=1.2$. h. Poisson, filled: $\lambda=5$; blank: $\lambda=10$. i. Binomial, filled: $n=20, p=0.25$; blank: $n=20, p=0.5$. 
There is always some probability of obtaining a negative value when sampling from a normal distribution. As such, it is not a purely accurate descriptor of parameters that cannot take negative values (e.g., daily ingestion of water, body weight). This may not be of concern if the mean of the distribution is located at least 5 standard deviations above zero, since then the likelihood of a negative value is extremely small, on the order of $3 \times 10^{-7}$ (three ten-millionths). If the mean is 6 standard deviations greater than zero, the likelihood of a negative value is about $1 \times 10^{-9}$ (one billionth). Keep in mind, however, that Monte Carlo analyses may use a large number of simulations, certainly on the order of tens of thousands. Consequently, there is actually a greater likelihood of sampling a negative value than one might realize. For example, if the probability of selecting a negative value in a single random sample is 0.0001 , then the likelihood of selecting at least one negative value in 10,000 random samples is about 0.63 . To circumvent this problem, analysts will sometimes use a truncated normal distribution, with truncation occurring at the smallest possible value, commonly zero.

5.2.2.2 Lognormal Distribution. A parameter, $x$, is said to be lognormally distributed when $y=\ln x$ is normally distributed. The lognormal distribution is commonly used to describe contaminant distributions and products of random variables. This distribution is descriptive of nonnegative, highly skewed quantities. It is particularly appropriate for representing large uncertainties that are expressed on a multiplicative or order-of-magnitude basis. For example, when the quantity $\mathrm{X}$ is known within a factor of 2 , or within an order-of-magnitude (factor of 10), a lognormal distribution is probably appropriate for its representation.

There are two, three, and four parameter lognormal distributions. We consider only the twoparameter lognormal here. The PDF for the twoparameter lognormal distribution is given by

$$
\begin{gathered}
f(x)=\frac{1}{x \sqrt{2 \pi \sigma_{y}^{2}}} \exp \left(-\frac{\left(\ln x-\mu_{y}\right)^{2}}{2 \sigma_{y}^{2}}\right) ; x>0, \\
-\infty \leq \mu_{y} \leq \infty, \sigma_{y}>0,
\end{gathered}
$$

where $\mu_{\mathrm{y}}$ and $\sigma_{\mathrm{y}}$ are the mean and variance of the transformed variable $y=\ln x$. These are the two parameters most commonly used to specify a lognormal distribution, though some authors will use the geometric mean $\left[\exp \left(\mu_{y}\right)\right]$ and geometric standard deviation $\left[\exp \left(\sigma_{y}\right)\right]$. There is no closed-form representation of the CDF, although it can be expressed in terms of the normal CDF.

5.2.2.3 Exponential Distribution. The exponential distribution is important in that it forms the basis for a number of other probability distributions. The exponential distribution has been used to describe the time until a specific event occurs (i.e., the interarrival time of a Poisson process) and is sometimes said to represent "pure death" or "survivalship" processes (Haimes et al. 1994). Examples include storm event durations, length of residence time, and spill sizes. The distribution has a single scale parameter, its shape and coefficient of variation are constant. The gamma and Weibull distributions are extensions of the exponential distribution that provide more flexibility in describing input parameters.

The exponential distribution is unique in that it is memoryless. As mentioned above, the exponential is commonly used to describe the time until an event occurs. It is memoryless because the conditional probability that the time until the event is $a+b$ units given that the event has not occurred in $a$ time units is the same as its initial probability of lasting $b$ time units.

The exponential PDF is given by $f(x)=\lambda e^{-\lambda x} ; x \geq 0, \lambda>0$.

The CDF for an exponentially distributed variable is given by

$F(x)=1-e^{-\lambda x} ; x \geq 0$.

5.2.2.4 Weibull Distribution. The Weibull distribution is a flexible distribution used in numerous engineering applications. Like the lognormal distribution, it is a non-negative distribution. The Veibull distribution is commonly used to model failure times in "weakest link" systems; it has also been used to model wind speed (Morgan 
and Henrion 1990) and spatial and temporal distributions of atmospheric radioactivity (Gilbert 1987). The estimate of $\gamma$ may be a reasonably good environmental "background" value, since $\gamma$ is a threshold parameter (Gilbert 1987).

The three-parameter Weibull (which reduces to the two-parameter Weibull when $\gamma=0$ ) PDF is given by

$$
\begin{gathered}
f(x)=\frac{\alpha}{\beta}\left(\frac{x-\gamma}{\beta}\right)^{a-1} \exp \left[-\left(\frac{x-\gamma}{\beta}\right)^{\alpha}\right]-\infty< \\
\gamma<\infty, x>\gamma, a>0, \beta>0,
\end{gathered}
$$

where $\gamma, \alpha$, and $\beta$ determine the location, shape, and scale, respectively, of the distribution. This reduces to the exponential distribution when $\alpha=1$ and $\gamma=0$. The CDF of the three-parameter Weibull is given by

$$
F(x)=1-\exp \left[-\left(\frac{x-\gamma}{\beta}\right)^{a}\right] \text {. }
$$

5.2.2.5 Gamma Distribution. The gamma distribution is most commonly used to describe the time until $k$ events occur in a Poisson process. It is similar to the lognormal, although it is less positively skewed and less tail-heavy (i.e., gives a lower probability to extreme values) than the lognormal. Gamma distributions have been used to represent precipitation quantities, pollutant concentrations, and the time between event occurrences when the event process has memory (e.g., there is event clustering) (Morgan and Henrion 1990). Gamma distributions have also been used to model interarrival times for multihit phenomena such as are theorized to exist in the process of carcinogenesis (Haimes et al.,1994).

The gamma distribution can be a two- or threeparameter distribution. The parameters in the three-parameter distributions, $\gamma, \alpha$, and $\beta$, determine the location, shape, and scale, respectively, of the distribution. When $\gamma=0$, the PDF reduces to the two-parameter gamma distribution. When $\alpha=1$, the gamma distribution is equivalent to the exponential distribution with parameter $1 / \beta$. The gamma distribution also characterizes the sum of independent and identically distributed exponential random variables. The PDF for the threeparameter gamma distribution is

$$
\begin{gathered}
f(x)=\frac{(x-\gamma)^{a-1}}{\beta^{a} \Gamma(a)} \exp \left[-\left(\frac{x-\gamma}{\beta}\right)\right]-\infty<\gamma \\
<\infty, x>\gamma, a>0, \beta>0 .
\end{gathered}
$$

The function $\Gamma(\mathrm{k})$ is the gamma function; when $\mathrm{k}$ is an integer, $\Gamma(k)=(k-1)(k-2)(k-3) \cdots(2)(1)$. There is no closed form for the CDF of the gamma distribution. When $\alpha \leq 1$, the PDF is L-shaped and is otherwise unimodal as shown in Figure 1.

5.2.2.6 Beta Distribution. Like the uniform and triangular distributions, the beta distribution has fixed endpoints. The distribution provides a flexible means for representing uncertainty within a finite range. The beta distribution has four parameters, $\alpha, \beta, \theta$, and $\gamma$. The endpoints are given by $\theta$ and $\gamma$. The shape and scale parameters are given by $\alpha$ and $\beta$, respectively.

The four-parameter beta PDF $[\operatorname{beta}(\alpha, \beta, \gamma, \theta)]$ is given by

$$
\begin{aligned}
f(x)= & \frac{\Gamma(a+\beta)}{\Gamma(\alpha) \Gamma(\beta)}(\theta-\gamma)^{1-a-\beta}(x-\gamma)^{a-1} \\
& (\theta-x)^{\beta-1} ; \gamma<x<\theta, a>0, \beta>0 .
\end{aligned}
$$

There is no closed form for the CDF. The PDF is U-shaped if $\alpha<1, \beta<1$, and J-shaped (or reverse $\mathrm{J}$-shaped) if $(\alpha-1)(\beta-1)<0$, and is otherwise unimodal.

5.2.2.7 Uniform Distribution. The uniform distribution is often used to describe parameters when there is a high degree of uncertainty. Like the beta distribution, its range is finite. It describes situations where there is an equally likely chance of any value in a finite range occurring. When not enough is known about a parameter to assess which values are more likely to occur, the uniform distribution is used. For example, a uniform distribution might be used to describe the probability of a puncture through a drum's surface at any location. 
The PDF of the uniform distribution is given by

$$
f(x)=\frac{1}{\beta-a} ; a<x<\beta .
$$

The CDF of the uniform distribution is given by

$$
F(x)=\left\{\begin{array}{cc}
0 & x \leq a, \\
\frac{x-a}{\beta-a} & a<x<\beta, \\
1 & x \geq \beta
\end{array}\right.
$$

As noted previously, there is some disagreement concerning the use of the uniform distribution as a representation of a highly uncertain parameter. Caution should be used in applying the uniform distribution. Occasionally a parameter may be described as "uniformly distributed between $10^{-3}$ and $10^{-5}$." A strict interpretation of this statement would imply that the probability that the value is between $10^{-3}$ and $10^{-4}$ is about 0.9 and the probability it is between $10^{-4}$ and $10^{-5}$ is 0.1 . More likely, the reference was to a log-uniform distribution that would assign an equal probability of 0.5 between $10^{-3}$ and $10^{-4}$ and between $10^{-4}$ and $10^{-5}$.

Haimes et al. (1994) recommend that the uniform and triangular distributions may work well when the relative error (ratio of largest value to smallest) is small, say less than a factor of 10 . When there is a large relative error, greater than a factor of 10 , then the loguniform and logtriangular are useful.

5.2.2.8 Triangular Distribution. This is another distribution commonly used when a great amount of uncertainty about the values of a parameter exists. Like the uniform, it is also has a finite range. It describes situations where it is believed that certain values have a higher likelihood of occurrence. Depending upon how the endpoints and mode are selected, the triangular distribution can result in a conservative approximation of truncated normal and lognormal distributions (Finley et al. 1994).

The triangular distribution has three parameters. The minimum and maximum are denoted by $\alpha$ and $\beta$, respectively. The mode (peak) is denoted by $\gamma$. The PDF of the triangular distribution is given by

$f(x)= \begin{cases}\frac{2(x-a)}{(\beta-a)(\gamma-\alpha)} & \alpha \leq x \leq \gamma \\ \frac{2(\beta-x)}{(\beta-\alpha)(\beta-\gamma)} & \gamma \leq x \leq \beta\end{cases}$

and its CDF is given by

$$
F(x)= \begin{cases}\frac{(x-a)^{2}}{(\beta-\alpha)(\gamma-\alpha)} & a \leq x \leq \gamma \\ 1-\frac{(\beta-x)^{2}}{(\beta-\alpha)(\beta-\gamma)} & \gamma \leq x \leq \beta\end{cases}
$$

5.2.2.9 Poisson Distribution. The Poisson distribution applies to discrete, non-negative valued variables. It is most commonly used to describe the number of events occurring in a fixed period of time. The distribution has a single parameter representing the mean of the distribution. When this parameter is relatively large, the Poisson distribution is typically approximated by a normal distribution.

The PMF for the Poisson distribution is given by

$f(x)=\frac{\lambda^{x} e^{-\lambda}}{x !} 0 \leq x<\infty, x$ integer, $\lambda>0$.

The CDF for the Poisson distribution is given by $F(x) \quad \sum_{i=0}^{x} \frac{\lambda^{i} e^{-\lambda}}{i !} 0 \leq x<\infty, x$ integer, $\lambda>0$.

5.2.2.10 Binomial Distribution. The binomial distribution also applies to discrete, nonnegative valued variables, and is commonly used to describe the number of events occurring in a fixed period of time or space. An example would be the number of days of measurable precipitation within a year at the INEL. The distribution has two parameters, $n$ and $p$.

The PMF of the binomial distribution is given by

$f(x)=\left(\begin{array}{l}n \\ x\end{array}\right) p^{x}(1-p)^{n-x}$, 
where $\left(\begin{array}{c}n \\ i\end{array}\right)$ is the number of combinations of $n$ objects taken $i$ at a time, $\frac{n !}{(n-i) ! i !}$. The binomial distribution $\mathrm{CDF}$ is given by

$F(x)=\sum_{i=0}^{x}\left(\begin{array}{l}n \\ i\end{array}\right) p^{i}(1-p)^{n-i}$.

5.2.3 Selecting Distributions. The risk analyst should use whatever a priori information on a parameter that is available. This may require a fair amount of effort, but is justifiable since parameter distribution selection is one of the more critical steps in the MCA process. The information may come from one of the three general sources: empirical, theoretical, and expert judgment.

There is no well-defined boundary between these sources of information; they are grouped together for convenience of discussion.

5.2.3.1 Empirical Sources. Empirical information includes observed data from site characterizations and controlled laboratory experiments. Sources include both site sampling results and data from the literature. Site characterization is always a preferred source of information, assuming the data is of reasonable quality. Data from controlled experiments will be subject to somewhat more uncertainty than site data because the experimental conditions may not be entirely representative of the site being assessed.

There are a variety of sources of information, depending upon the parameter type. Contaminant concentrations can result from sampling programs at the site, transport models, process data, and historic data. Slope factors and hazard indexes are developed by the EPA and regularly updated in the Health Effects Assessment Summary Tables (HEAST) and IRIS databases. These databases commonly provide relevant references to the literature that generated the estimate. Since the EPA does not specify distributions for slope factors and hazard indexes, the literature can potentially be useful in developing distributions. Pathway parameters and transfer coefficient distributions may be obtained from sampling, the literature, and theory. Demographic statistics are generated and reported by various government agencies, in particular commerce departments and health departments.

A distribution can be fit to empirical data using the following steps:

1. Plot a histogram of the data.

2. Roughly determine which of the distributions discussed in Section 5.2.2 and shown in Figure 1 best represent the histogram of observed values.

3. Determine estimates of the parameters of the distribution. For example, for the normal distribution you estimate the mean and variance. For the more obscure distributions, the references cited in Section 5.2.2 should be consulted for methods of estimating the distribution's parameters.

4. Implement either a probability plot or a goodness of fit test. Probability plots plot the quantiles of the observed distribution against the quantiles of the assumed distribution. If the assumed distribution is a good fit to the observed data, the plot will look like a straight line on the diagonal. Goodness of fit tests for distributions include the $\mathrm{X}^{2}$ test and Kolmogorov-Sminov tests. A discussion on their use can be found in most introductory statistics texts.

5. If the assumed distribution does not appear to fit the data well, try other distribution types. If none of the distribution types fit the data well, it may be necessary to use bootstrapping methods in the MCA (i.e., sampling from the observed data); use advanced methods for fitting density functions (e.g., splines), or consult other sources of data (theory and expert judgment).

There has also been work done on developing standard distributions; that is, distributions which should be the same for any human health risk assessment. Examples of parameters that might be standardized are body weight and residence time. Brorby and Finley (1994) propose some standard 
distributions summarized in Table 4. In addition to the distributions shown in Table 4, Finley et al. (1994) propose standard distributions for skin area by body part, total water intake, total fish consumption, time since last job change, and time in a shower. Finley et al. (1994) also break many of the distributions down by age. This is useful in that breaking the MCA down into ages removes some unwanted correlations or contradictory sample selections (e.g., a five-year-old weighing $180 \mathrm{lb}$ ). In some cases, results discussed in the Exposure Factors Handbook (EPA 1989b) might be useful in developing distributions. There is also a body of literature generated by the nuclear industry for safety PRAs that may be useful in developing distributions.

5.2.3.2 Theory. Scientific results on a variety of phenomena may provide information about parameter distributions, and may be preferred over empirical sources, particularly if the quality of the empirical data is questionable. For example, the decay of radionuclides is assumed to follow a Poisson distribution; while instruments for the detection and measurement of decay are quite sensitive, if the observed data were from certain continuous monitors that are not precise, the use of the Poisson distribution may be preferred over using observed data since the observed data may be of such low quality.

One could also refer to the Central Limit Theorem to help develop distributions, as discussed in Seiler and Alvarez (1994). From this Theorem, it can be inferred that if the variation in a random variable results from the sum of errors of other variables, the resulting variable will tend toward a normal distribution. And since the logarithm of a product is a sum, variables resulting from products of other random variables will tend toward a lognormal distribution.

It is preferable to use theories that are well established and accepted by the scientific community when using theory to develop parameter distributions. Controversial theories should be avoided. If there are conflicting theories relating to a parameter's distribution, it may be necessary to treat the parameter through switchover or parametric methods rather than probabilistically.

5.2.3.3 Expert Judgment. Expert judgment should only be used to develop parameter distributions as a last resort. There are some fairly advanced methods for eliciting expert opinion discussed in Morgan and Henrion (1990) and Rish (1988). The EPA (1994) suggests that when expert opinion is used, the resulting parameter should only be modeled as a uniform or triangular distribution because of the large degree of uncertainty. As previously discussed in Section 5.2.2, Seiler and Alvarez (1994) present a strong argument against this use of these "simple" distributions.

\subsection{Monte Carlo Sampling}

Monte Carlo methods provide a conceptually and computationally simple method for quantitatively estimating the uncertainty in the risk or exposure. In crude Monte Carlo analysis, a value is drawn at random from the distribution for each input parameter in the risk or exposure equation. Together this set of random values is used to calculate the risk or exposure. The entire process is repeated $n$ times, producing $n$ independent samples with corresponding risks or exposures. The resulting set of $n$ risks or exposures form an uncertainty distribution for the risk or exposure.

Latin Hypercube Sampling is a Monte Carlo method which is more efficient than crude Monte Carlo sampling. Rather than using a completely random sampling scheme, Latin Hypercube Sampling uses a stratified sampling scheme to ensure more efficient coverage of the input parameter uncertainty' distributions.

To implement Monte Carlo sampling, the number of samples required to achieve a given level of precision in the risk estimates must be selected. It is also necessary to determine the software requirements and quality of the results. These issues will be discussed in this section.

\subsubsection{Determine the Number of Monte} Carlo Salmples. The determination of the number of samples should be approached through the 
Table 4. Standard probability density functions proposed by Brorby and Finley (1994).

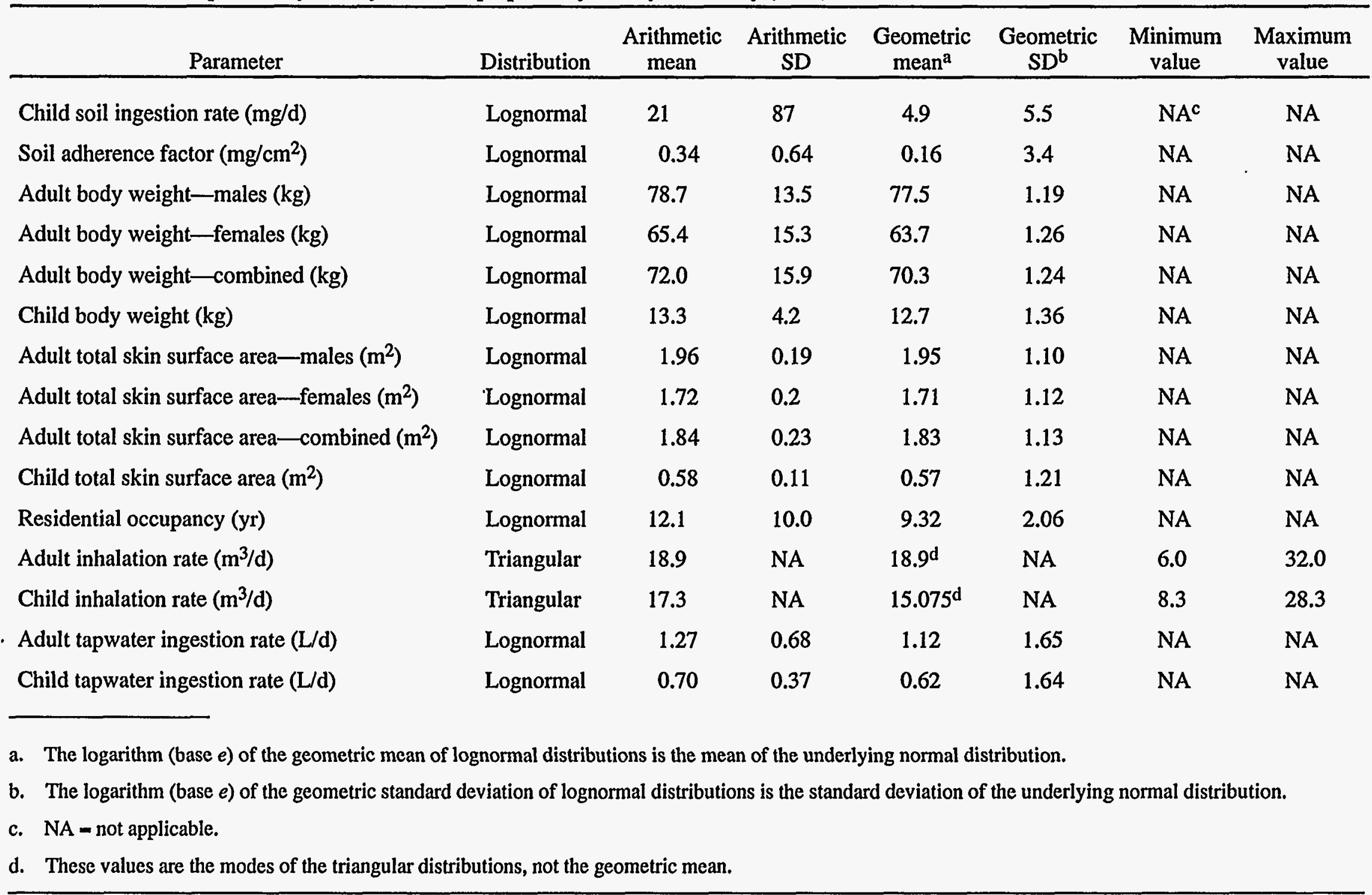


data quality objectives. Specifically, it is recommended that the precision of the estimation of the median risk be controlled. This will result in a conservative (i.e., relatively large) number of samples since it is more conservative than estimating an upper percentile of the risk distribution with the same precision.

The required sample size, $n$, for estimating the $p$ th percentile with a given level of absolute precision, $\Delta p$, is given by

$n=p(1-p)\left(\frac{z_{a}}{\Delta p}\right)^{2}$,

where $z_{\alpha}$ is the quantile of the standard normal distribution corresponding to the $1-(\alpha / 2)$ confidence level.

For the median, $p=0.5$. When $\alpha=0.05$ and $\Delta p=0.01$, the sample size is

$n=0.5(1-0.5)\left(\frac{2}{0.01}\right)^{2}=10,000$.

This is roughly the number of samples typically recommended (e.g., Burmaster and Anderson 1993; McKone and Bogen 1991). However, Morgan and Henrion (1990) note that this level of precision may be unwarranted given the empirical uncertainty in developing the input parameter distributions. They recommend simulations on the order of hundreds rather than thousands.

Others (e.g., Burmaster and Anderson 1993) argue that large numbers of samples are needed to estimate the tails of the distribution, which would seem to contradict the sample size equation given above. In the equation given above, $\Delta p$ is a fixed absolute precision and, as can be seen from the sample size formula, the extreme tails of the distribution (e.g., $p=0.01$ ) require far fewer samples to achieve the same level of precision for percentiles near the median. However, the relative precision of the estimates of the $p$ th percentiles in the tails is much worse than the relative precision of the median estimate.
If the number of simulations required is determined to be excessive due to computing requirements or other limitations, the best option is to consider using LHS. In a typical MCA, sampling from the input distributions is completely random; in LHS, stratified sampling is used. Essentially, in LHS each input parameter distribution is broken into $n$ strata, each having equal probability of $1 / n$ (see Figure 4). A value is randomly selected from each stratum and randomly matched with values from the other input parameters to form the input for a single simulation run. Not only does the technique reduce the number of runs, it also can be used to ensure sampling from the tails of the input distributions. Iman and Helton (1985) provide a fuller discussion of LHS and have a Fortran routine available for its implementation. Since it is a common alternative method of sampling, it is available in commercial software packages as well.

Actually, there does not appear to be any reason for not using LHS techniques in place of the nonstratified MCA methodology. LHS is more efficient because it provides a more precise risk estimation in fewer runs. The only time LHS may run into problems is when there is periodicity to the distribution, as discussed in Morgan and Henrion (1990). However, for the types of parameters considered in environmental and human health risk assessment, this behavior is extremely unlikely.

5.3.2 Assess Quality of the Results. Upon completion of the simulation (here, a simulation is considered to be a fixed set of samples and their results), the quality of the results should be assessed. Two characteristics should be assessed: reproducibility and uncertainty importance. The reproducibility criterion checks whether the same results (within a stated level of precision) would be produced if the simulation were run again under the same assumptions. The uncertainty importance assesses how much the variation in the input parameters influences the variation in the risk.

5.3.2.1 Reproducibility. Assessment of the reproducibility of the results is a check that enough samples were selected for the final risk distribution 

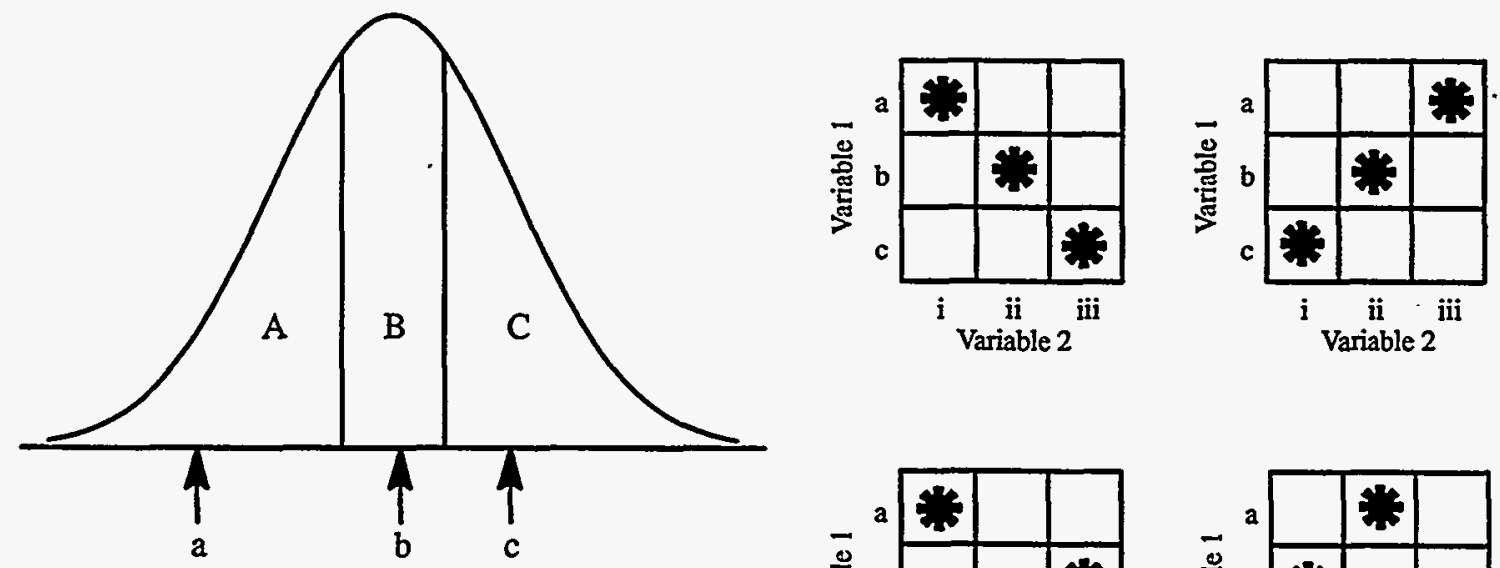

Step 1. From the first variable, tandomly select $a$ value $(a, b, c)$ from each equal probability region (A, B, C).
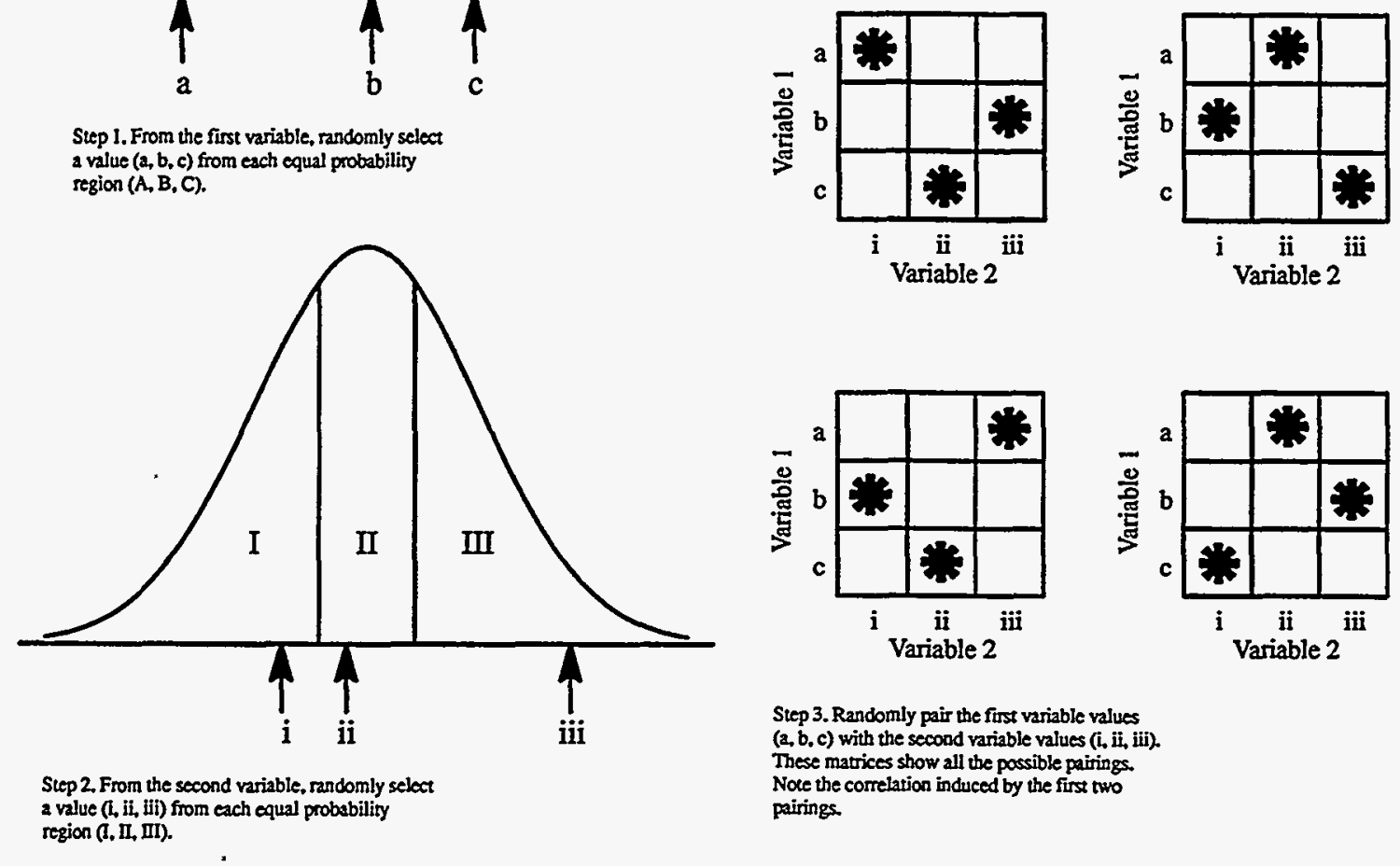

Step 3. Randomly pair the first variable values (a,b, c) with the second variable values (i, ii, iii). These matrices show all the possible pairings.

Note the correlation induced by the first two

pairings.

Figure 4. Example of LHS, with two variables and three samples. The two input variables are normally distributed. Note that regions $A, B$, and $C$ in the first variable and I, II, and III in the second variable each account for one third of the area under the curve.

to converge. That is, if the simulation is run again, the same risk distribution will result (within a stated level of precision). The best way to assess reproducibility is to run another simulation of the same size (i.e., same number of samples) and compare the results with the original simulation. The comparison can be done using a KolmogorovSmirnov test or other related $\mathrm{X}^{2}$ goodness of fit tests. Alternatively, certain summary statistics of the resulting distributions could be compared; for example, the means, variances, and selected percentiles (e.g., 50th, 95th). Thompson et al. (1992) use this latter method by computing the relative difference between the results. They felt the distributions to be reproducible if the summary statistics had relative differences of less than $1 \%$.

When comparing summary statistics, the extreme values (i.e., those in the tails of the distributions) are likely to be fairly sensitive; particularly the minimum and maximum. Less extreme percentiles in the tails of the distribution can also be affected. For example, Thompson et al. (1992) note that when they repeated a simulation of 10,000 samples, the 95 th percentile was not quite 
within $1 \%$ of the original simulation's estimate of the 95 th percentile.

\subsubsection{Uncertainty Importance. Asses-} sing the uncertainty importance is a process of attributing/apportioning the uncertainty in the risk to the input parameters. By so doing, one can determine which input parameters most affect the final uncertainty and may take steps to reduce the uncertainty in the input parameter. A number of methods for apportioning the uncertainty are available. It is recommended that at a minimum, correlation coefficients between each input parameter and the risk be determined.

To check the uncertainty importance of the input variables, the individual samples (i.e., the randomly selected values from each input parameter for each simulation with the resulting risk) must be saved. This allows the analyst to check that the sampling distributions were appropriately generated (e.g., the samples from a normally distributed input parameter with mean $\mu$ and standard deviation $\sigma$ look like a normal distribution with mean $\mu$ and standard deviation $\sigma$ ).

The Pearson correlation coefficient between the input parameter $x$ and the risk $y$ is given by

$$
\hat{\varrho}=\frac{\operatorname{Cov}(x, y)}{\sqrt{\operatorname{Var}(x) \operatorname{Var}(y)}}=\frac{\sum_{i=1}^{n}\left(x_{i}-\bar{x}\right)\left(y_{i}-\bar{y}\right)}{\sqrt{\sum_{i=1}^{n}\left(x_{i}-\bar{x}\right)^{2} \sum_{i=1}^{n}\left(y_{i}-\bar{y}\right)^{2}}},
$$

where the subscript $i$ refers to the $i$ th simulation, $\operatorname{Cov}(x, y)$ is the covariance between $x$ and $y, \operatorname{Var}(x)$ is the variance of $x$, and $\operatorname{Var}(y)$ is the variance of $y$. The correlation gives an estimate of the linear contribution of each input parameter to the uncertainty in the risk. It is inherently a global measure of uncertainty importance, averaging the effect of each input over the joint probability distribution for all other inputs (Morgan and Henrion 1990).

The correlation coefficient indicates linear relationships. If the relationship is non-linear, or either the input distribution or the risk distribution is highly skewed or has heavy tails, or there are extreme outliers, alternatives to the correlation coefficient should be considered. Probably the best alternative in these situations is to use a rank correlation (i.e., calculate the correlations between the ranks of the input parameters and the risk) such as "Spearman's $\rho$ " (Steel and Torrie 1980). Regardless of the correlation method used, it is also useful to plot each input parameter against the risk results to see if there are clear patterns in the relationships.

\subsubsection{Implementation of the Monte Carlo} Simulation. The actual implementation of the Monte Carlo simulation will be determined by the software used. Some of the commercially available programs allow specification of the parameter input distributions, the risk equation, and the number of runs. They produce summaries of the results, including the graphics discussed in Section 5.4.

The MCA sampling should reflect correlation between the input parameters, particularly if that correlation has an absolute value greater than 0.6. As discussed in Iman and Conover (1985), correlation is difficult to implement in MCA, particularly when the input parameters follow different distributional forms (e.g., lognormal, binomial). They propose a method for using rank correlation in place of linear correlation. Rank correlation is essentially the linear comelation of the ranks of the input parameters. This method has been implemented in a number of commercial MCA packages.

Because of the large sample sizes commonly involved in Monte Carlo analyses, attention must be paid to the quality of the random number generator. Computer random number generators are actually based on deterministic equations; however, these equations are such that the length of the sequence is long and the pattern of the pseudorandom numbers appears to be random. There have been cases where widely used generators introduce undesirable correlations and patterns into the samples. Knuth (1969) has a good discussion of random number generators and tests of the quality of the generators.

In some cases, it may be easier to break the simulation into parts. For example, if there is a large transport model that is too time-consuming to run 
upwards of 10,000 times, the distribution of the output from the transport model may be determined through other means (e.g., LHS); this output distribution could then be entered into the risk model. For example, if the transport model is used to determine the contaminant concentration at the receptor, the distribution of the concentration could be determined from using LHS on the input parameters to the transport model. The resulting concentration distribution could then be entered into the risk equation MCA. This method should not be implemented if the input parameters used in the separate model are strongly correlated with those used in the final model.

\subsection{Presentation of Results}

It is recommended that the resulting risk distributions be presented as both a frequency distribution (histogram) and cumulative distribution with the calculated BRA point estimate clearly identified.

The following should be included in the presentation of results:

1. A plot of the frequency distribution (histogram) of the resulting risk. The BRA point estimate should be clearly identified on the plot.
2. A plot of the cumulative distribution of the resulting risk. The BRA point estimate should be clearly identified on the plot.

3. A summary table of the resulting risks; this table should include the mean, standard deviation, number of runs, minimum, maximum, and the icosatiles (i.e., every 5 th percentile) of the distribution.

4. A summary table presenting the correlation coefficients between the input parameters and the resulting risk.

5. For each input parameter distribution used in the simulations, present the PDF (graphically) and a justification for the use of the distribution. Note the location of the point estimate of the parameter used in the BRA.

6. Clearly identify which uncertainties are quantified and which are left unquantified (e.g., model uncertainty).

7. A discussion of the assumptions and qualitative uncertainties. This will include discussions of models used. The analyst should note which assumptions are conservative and which are not. 


\section{EXAMPLE MONTE CARLO ANALYSIS}

The following section presents a hypothetical example highlighting the MCA steps as presented in this report. It is loosely based on an example given in Thompson et al. (1992). In this example, a soil is contaminated with benzene, a Group A carcinogen (i.e., a known human carcinogen). The example considers only a residential scenario adult ingestion exposure. Under this scenario, a person lives at the site where the benzene was spilled. They may ingest the benzene from dirty hands, inhalation of particulates with subsequent ingestion of the particulates trapped in the throat, etc. Results from this example MCA analysis are intended for illustrative purposes only.

The equations used to calculate the risk for both the point estimate and MCA results are from EPA (1989a). The intake (mg/kg-day) via ingestion of chemicals in soil is given by

Intake $(\mathrm{mg} / \mathrm{kg}$-day $)=\frac{\mathrm{CS} \times \mathrm{IR} \times \mathrm{CF} \times \mathrm{FI} \times \mathrm{EF} \times \mathrm{ED}}{\mathrm{BW} \times \mathrm{AT}}$,

and the carcinogenic risk is given by

Risk $=$ Intake $\times \mathrm{SF}$,

where symbol definitions are given in Table 5.

\subsection{BRA Point Estimate}

As discussed previously, the BRA point estimate uses a mixture of conservative and average estimates of the input parameters in the risk model. The values used for the point estimate in this example are shown in Table 5. The source of the value for each parameter in the model is also identified in Table 5. These values are entered into the equation given above to get the BRA point estimate.

Initial distributions for some of the parameters are also given in Table 5. It is not necessary to estimate parameter distributions when calculating BRA point estimates of risk. These distributions were used to determine the location of the point estimate in the distribution. This allows some degree of the assessment of the conservatism of the estimate. How these distributions were selected are discussed in Section 6.3.

The point estimate of risk for this example is calculated as the intake multiplied by the slope factor (SF) for benzene, $2.9 \times 10^{-2}(\mathrm{~kg}-\mathrm{d}) / \mathrm{mg}$. Using the values given in Table 5 , the intake is estimated to be $3.4 \times 10^{-4} \mathrm{mg} /(\mathrm{kg}$-day). The point estimate of risk is therefore $1 \times 10^{-5}$.

A risk value of $1 \times 10^{-5}$ is a borderline acceptable risk, falling closer to the lower end acceptable risk of $1 \times 10^{-6}$ than the upper end acceptable risk of $1 \times 10^{-4}$. Hence it is reasonable to pursue an MCA in this example to clarify the uncertainties and conservatism in the point estimate.

\subsection{Initial Sensitivity Analysis}

The first step before implementing an MCA is identifying the parameters that most impact the risk. Those parameters having the largest effect on the risk should be treated probabilistically in the MCA. Focusing attention on these parameters will provide the most benefit.

To determine which parameters are most important, a nominal range method analysis is used. Essentially each parameter is varied, one at a time, and the risk calculated while holding the remaining parameters at their approximate median. The range of the risk values calculated for each parameter are then ordered from largest to smallest-the larger ranges having more impact on the risk.

For each parameter, a lower limit, median, and upper limit are determined. In this example the lower limit was chosen to be greater than no more than 5\% of the parameter's distribution; the upper limit was chosen to be greater than at least $95 \%$ of the parameter's distribution. At this point it is not important to know the exact range of the parameter; rather, a reasonable estimate is required. The selected ranges for the parameters in this example are shown in Table 6. 
Table 5. Variables and constants used in the benzene-contaminated soil ingestion scenario.

\begin{tabular}{|c|c|c|c|c|c|c|}
\hline Parameter name & Symbol & Units & $\begin{array}{c}\text { Point } \\
\text { estimate }\end{array}$ & Distribution ${ }^{b}$ & $\begin{array}{l}\text { Distribution } \\
\text { referencec }\end{array}$ & $\begin{array}{l}\text { Point estimate } \\
\text { location }\end{array}$ \\
\hline Body weight & BW & $\mathrm{kg}$ & 70 & $\begin{array}{l}\text { Female: lognormal }(4.96,0.20)^{\mathbf{d}} \\
\text { Male: lognormal }(5.13,0.17)\end{array}$ & Brainard et al. 1992 & $\begin{array}{l}65 \text { th \%ile } \\
30 \text { th \%ile }\end{array}$ \\
\hline Ingestion rate & $\mathbb{R}$ & mg soil/d & 100 & Lognormal $(2,1.7)$ & Brorby et al. 1994 & 94th \%ile \\
\hline Exposure frequency & EF & $d / y r$ & 365 & & & \\
\hline Exposure duration & ED & $\mathrm{yr}$ & 30 & Lognormal $(0.6,1.4)$ & Israeli et al. 1992 & 98th File \\
\hline Averaging time & $\mathrm{AT}$ & d & 25,550 & Beta(4.2,2.1,5475,36500) & Wright 1993 & 42nd \%ile \\
\hline Fraction ingested & FI & Unitless & 1.0 & & & \\
\hline Conversion factor & CF & $\mathrm{kg} / \mathrm{mg}$ & $1 \times 10^{-6}$ & & & \\
\hline $\begin{array}{l}\text { Benzene concentration } \\
\text { in soil }\end{array}$ & CS & $\mathrm{mg} / \mathrm{kg}$ & 550 & Lognormal $(1.7,2.7)$ & Calculated & Max.e \\
\hline Slope factor & SF & $(\mathrm{kg} \bullet \mathrm{d}) / \mathrm{mg}$ & $2.9 \times 10^{-2}$ & Lognormal $(-4.33,0.67)$ & Thompson et al. 1992 & 88th \%ile \\
\hline \multicolumn{7}{|c|}{$\begin{array}{l}\text { a. Except for CS, CF and SF, point estimates come from EPA (1989a). The SF is from HEAST (EPA, 1991). The CS is calculated from soil sample } \\
\text { results. }\end{array}$} \\
\hline \multicolumn{7}{|c|}{$\begin{array}{l}\text { b. The lognormal distribution parameters are the mean and standard deviation of the log-transformed data. The beta distribution parameters are the } \\
\text { two shape parameters followed by the minimum and maximum. }\end{array}$} \\
\hline \multicolumn{7}{|c|}{ c. See discussion in Section 6.3 on parameter distribution selections. } \\
\hline \multicolumn{7}{|c|}{ d. The lognormal distribution for body weight is in units of pounds. A conversion factor is added to the risk equation to convert to kilograms. } \\
\hline
\end{tabular}

Table 6. Input values and calculated risks for the nominal range method sensitivity analysis.

\begin{tabular}{|c|c|c|c|c|c|c|c|}
\hline \multirow[b]{2}{*}{ Parameter name } & \multirow[b]{2}{*}{ Symbol } & \multirow[b]{2}{*}{ Units } & \multicolumn{3}{|c|}{ Parameter range } & \multicolumn{2}{|c|}{ Calculated risk } \\
\hline & & & Median & Lower & Upper & Low & High \\
\hline Body weight & BW & $\mathrm{kg}$ & 67 & 48 & 94 & $2.5 \times 10^{-9}$ & $4.9 \times 10^{-9}$ \\
\hline Ingestion rate & IR & mg soil/d & 31 & 8 & 116 & $9.1 \times 10^{-10}$ & $1.3 \times 10^{-8}$ \\
\hline Exposure frequency & $\mathrm{EF}$ & $d / y r$ & 340 & 300 & 365 & $3.1 \times 10^{-9}$ & $3.8 \times 10^{-9}$ \\
\hline Exposure duration & ED & yr & 10 & 3 & 40 & $1.1 \times 10^{-9}$ & $1.4 \times 10^{-8}$ \\
\hline Averaging time & AT & $d$ & 25,550 & 10,950 & 34,675 & $2.6 \times 10^{-9}$ & $8.2 \times 10^{-9}$ \\
\hline Fraction ingested & FI & Unitless & 0.8 & 0.1 & 1.0 & $4.4 \times 10^{-10}$ & $4.4 \times 10^{-9}$ \\
\hline $\begin{array}{l}\text { Benzene concentration } \\
\text { in soil }\end{array}$ & Cs & $\mathrm{mg} / \mathrm{kg}$ & 5.5 & 0.05 & 550 & $3.2 \times 10^{-11}$ & $3.5 \times 10^{-7}$ \\
\hline Slope factor & SF & $(\mathrm{kg}-\mathrm{d}) / \mathrm{mg}$ & 0.013 & 0.004 & 0.04 & $1.1 \times 10^{-9}$ & $1.1 \times 10^{-8}$ \\
\hline
\end{tabular}


The estimated risk when all the parameters are held at their median value is $3.5 \times 10^{-9}$. Results from varying parameters one at a time are shown in Table 6 . The ordered swing weights (differences between the low and high risks) from the nominal range analysis (see Section 5.1.1) are shown in Table 7.

Table 7. Rank of parameter importance in the risk equation as determined by the nominal range method.

\begin{tabular}{clr}
\hline Rank & \multicolumn{1}{c}{ Parameter name } & $\begin{array}{r}\text { Swing } \\
\text { weight } \\
\left(\times 10^{-9}\right)\end{array}$ \\
\hline & & \\
1 & $\begin{array}{l}\text { Benzene concentration } \\
\text { in soil }\end{array}$ & 352.2 \\
2 & Exposure duration & 13.0 \\
3 & Ingestion rate & 12.3 \\
4 & Slope factor & 9.8 \\
5 & Averaging time & 5.6 \\
6 & Fraction ingested & 4.0 \\
7 & Body weight & 2.4 \\
8 & Exposure frequency & 0.6 \\
\hline
\end{tabular}

Four levels of parameter importance appear to emerge from these results. Benzene concentration in soil is clearly has the most influence on the risk estimates. Exposure duration, ingestion rate, and slope factor form the next level of importance. Averaging time, fraction ingested, and body weight have a moderate influence on the risk. Exposure frequency has relatively little effect on risk.

This grouping indicates that most of the effort should be spent in quantifying the uncertainty due to benzene concentration in soil. It would also be reasonable to expend some time on the second group of parameters (exposure duration, ingestion rate, and slope factor). Less is to be gained from effort spent developing a distribution for the remaining parameters. These conclusions assume there is no significant interaction or correlation between the parameters.

In this example, because of the simplicity of the model, a more quantitative analysis of the importance of the individual parameters can be implemented. By taking the logarithm (base 10) of both sides of the risk equation given in the introduction to Section 6 , one can roughly quantify the proportion of the variance in $\log$ (risk) due to each of the log-transformed input parameters. The uncertainty in the soil concentration can change $\log$ (risk) by $4.04\left[\log \left(C S_{\max }\right)-\log \left(\mathrm{CS}_{\min }\right)\right]$ units. Similarly, the change in log(risk) due to ED, IR, SF, AT, FI, $\mathrm{BW}$, and $\mathrm{EF}$ is $1.12,1.16,1,0.50,1,0.29$, and 0.09 , respectively. These values can be considered multiples of the standard deviation of the $\log$ (risk). Assuming independence of the input parameters

[so that the total log(risk) variance is the sum of the input parameter variances], the total sum of the squared changes in $\log$ (risk) is 21.24 . Hence the percentage of total variance in $\log$ (risk) due to $C S$, ED, IR, SF, AT, FI, BW, and EF is $77,6,6,5,1,5$, $<1$, and $<1$, respectively.

Note that this analysis does give a somewhat - different ordering than the nominal range method. This is because in the nominal range method, the importance of a particular parameter partly depends on the medians of the remaining parameters-which is not the case in the rough variance component analysis done above. Both sets of results clearly indicate that soil concentration contributes the most to the uncertainty in the risk estimates.

\subsection{Parameter Distribution Selection}

Selection of the parameter distributions is the most important step in the MCA. The resulting risk distribution/uncertainty depends solely on the selected input parameter distributions (for a given model). Also, selection of parameter distributions can be a subjective process and subject to error or misunderstanding. While many parameter distributions can be generated by someone with general knowledge, certain parameters, such as slope factors, generally require professional expertise. 
The following sections discuss how the parameter distributions shown in Table 5 were selected. In some cases, the distributions were taken directly from the literature; in other cases, distributions were fit to observed data. In one case, fraction ingested, no information was readily available. Since this parameter has only moderate influence on risk, it will be treated as a constant (in this case, 1). Also, while there is information on amount of time spent at home (see Price et al. 1992), it was not in a form for easy distribution fitting; since exposure frequency has a minor impact on the risk, it too will be treated as a constant. While body weight is not considered important, it will be treated probabilistically in the Monte Carlo analysis, only because the distribution is widely and easily available and does not add any significant effort to the analysis. Had its distribution, like those of fraction ingested and exposure frequency, been difficult to assess, body weight would not have been included probabilistically.

Because the distributions used in this example are based on nationwide statistics, an assumption is being made that the demographics of the area contaminated is in some sense a cross section of United States. Had it been indicated that the example scenario occurs in a school yard or nonresidential area, the distributions (and potentially the exposure model) would be different.

6.3.1 Benzene Concentration in Soil. A total of 20 soil samples were randomly selected at the site; concentrations of benzene in those samples ranged from 0.05 to $550 \mathrm{mg} / \mathrm{kg}$. The results were clearly from a lognormal distribution. Figure 5 shows the empirical cumulative distribution function and the fitted lognormal cumulative distribution function. Since the observed and fitted values differ by less than the Kolmogorov-Smirnov difference (shown in Figure 5) the fitted distribution is not unreasonable given the observed values.

Further data analysis indicates that the benzene concentrations in the soil be modeled as a lognormal distribution with underlying normal distribution parameters of 1.7 for the mean and 2.7 for the

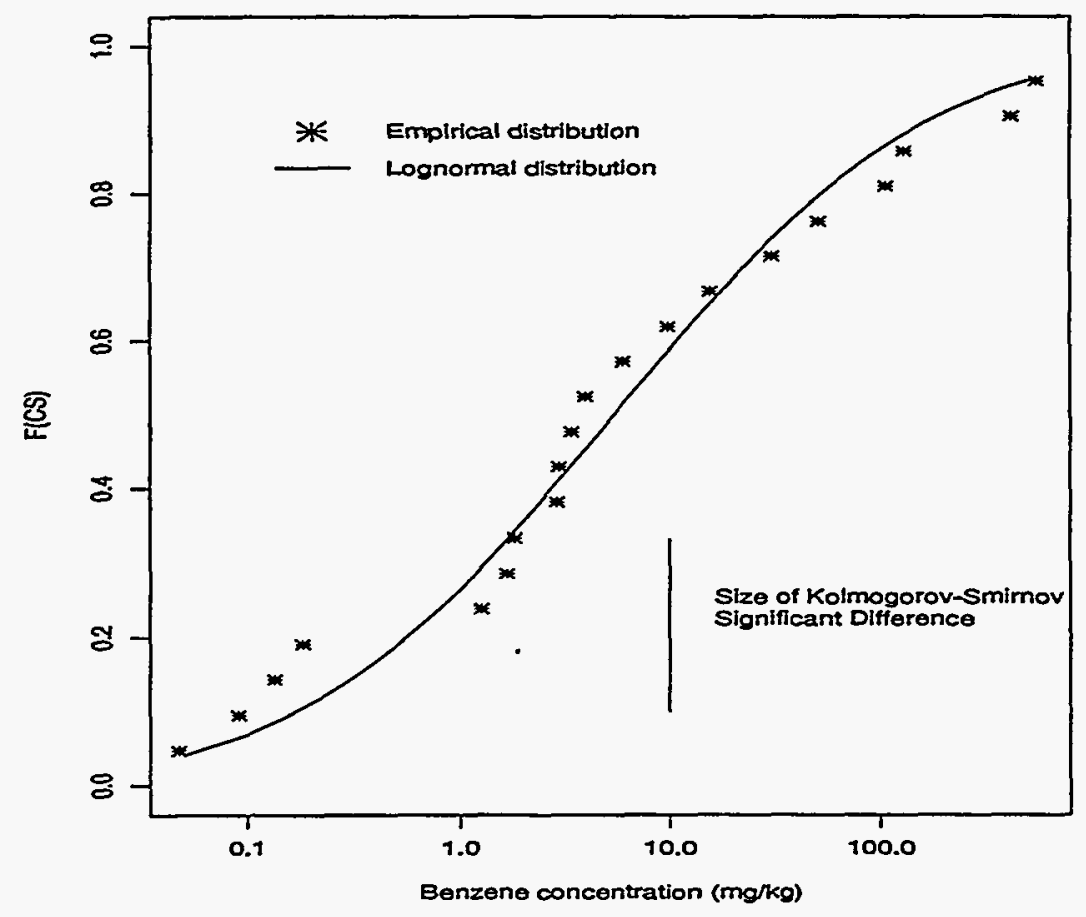

Figure 5. Fit of a lognormal distribution to benzene concentration in soil. The size of the KolmogorovSmimov indicates the vertical distance between the observed value and fitted distribution that would be considered significant at $\alpha=0.10$. 
standard deviation. This distribution is shown in Figure 6 . The $550 \mathrm{mg} / \mathrm{kg}$ concentration used in the BRA point estimate is at about the 96 th percentile of this distribution, as illustrated in Figure 6.

Since benzene concentration is the most influential parameter in the risk equation, the quality of the available data should be assessed. In particular, the quality of the mean and variance estimates of the lognormal distribution would be assessed using standard statistical techniques, such as confidence intervals. If the variability of the mean and variance estimates are large enough, further soil sampling for benzene concentrations may be warranted.

6.3.2 Exposure Duration. The exposure duration is the time that a person resides at the contaminated location. The EPA (1989b-Exposure Factors Handbook) reports that the 90th percentile for exposure duration is 30 years and the median is 9 years. These values are based on a survey conducted by the U.S. Bureau of the Census in 1983. The values represent the amount of time the householder live in their current house.
Israeli and Nelson (1992) point out that using time spent in the current house is biased high for the total residence time. That is, total residence time and current residence time do not generally share the same distribution. This is because the majority of households a typical person lives in are for relatively short periods, but may spend most of the time at one or two residences. Additionally, Israeli and Nelson (1992) note that in averaging total residence times over a time interval, frequent movers may appear several times, while in averaging current residence times each household appears only once. For the risk assessment, the total residence time is of more interest.

Israeli and Nelson (1992) develop a model for total residence time based on current residence time data collected from two large surveys of U.S. households. They find that the average total residence time calculated for all U.S. households is 4.6 years. Based on the results presented in Figure 3 of Israeli and Nelson (1992), the distribution for the total residence time is highly skewed right, with over $50 \%$ of the population having a residence time of less than 3 years at a given house.

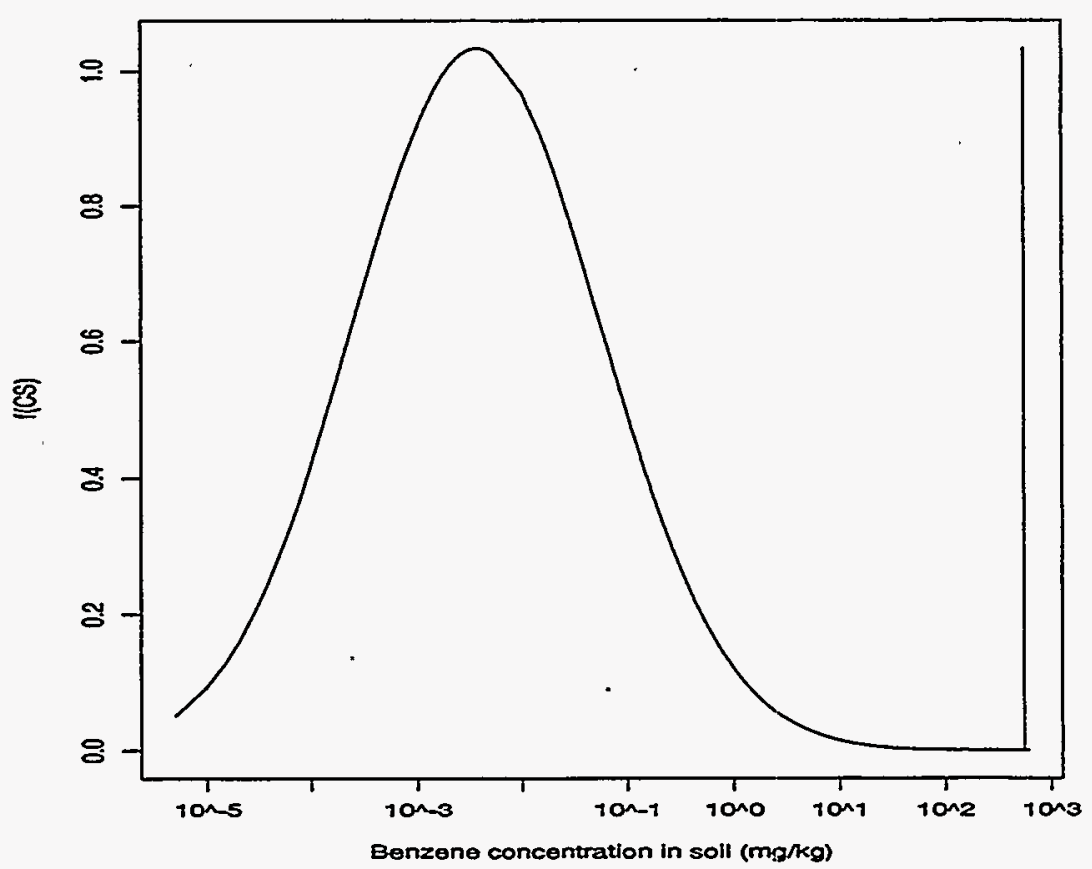

Figure 6. Probability density function for benzene concentration in soil. The vertical line indicates the concentration value used in the BRA point estimate. 
A lognormal distribution with mean 0.546 and standard deviation 1.43 (on the log scale) provided a good fit to the total residence time curve from Figure 3 of Israeli and Nelson (1992). The observed and fitted values are shown in Figure 7. The fitted distribution passes the KolmogorovSmimov one-sample test (at $\alpha=0.10$ ), indicating that the distribution is a reasonable fit to the data. The PDF is shown in Figure 8. The suggested EPA recommended estimate of 30 years falls at the 98 th percentile; EPA's recommended median of 9 years falls at the 88 th percentile. These values are identified in Figure 8.

6.3.3 Ingestion Rate. The ingestion rate is the amount of soil ingested in a given day. Soil ingestion usually occurs incidentally as a result of hand to mouth contact, though pica behavior may result in abnormally high ingestion amounts. For adults, the main two soil ingestion routes are inhalation (of dust or wind-blown soil), with subsequent ingestion of soil trapped in mucous membranes, and adhesion of soil on hands, which are then placed near the mouth during smoking or eating.
Most of the soil ingestion rate data focuses on children; data for adult soil ingestion is lacking. The EPA Exposure Handbook (EPA, 1989b) declines to recommend a soil ingestion rate for adults because of the lack of data. However, it does cite a study by Calabrese from 1987 (apparently reported in Calabrese et al., 1989) that estimates adults ingest $1-100 \mathrm{mg} /$ day with an "intermediate" value of $10 \mathrm{mg} / \mathrm{day}$. In later guidance for soil ingestion, EPA (1989c) recommends an ingestion rate of $100 \mathrm{mg} /$ day for age groups greater than 6 years old.

LaGoy (1987) takes the results from two previous studies and estimates that adults on average ingest $50 \mathrm{mg} /$ day of soil, though it is noted that a value of $25 \mathrm{mg} /$ day may be a better estimate for adults who do not exhibit frequent hand-to-mouth behavior. Thompson and Burmaster (1991) develop a lognormal distribution (with an average of $91 \mathrm{mg} /$ day) of soil ingestion by children based on some of the same data used by LaGoy (1987). Brorby and Finley (1994) also develop a lognormal distribution for soil ingestion by children, but they find the average to be $21 \mathrm{mg} /$ day and base their results on the Calabrese data.

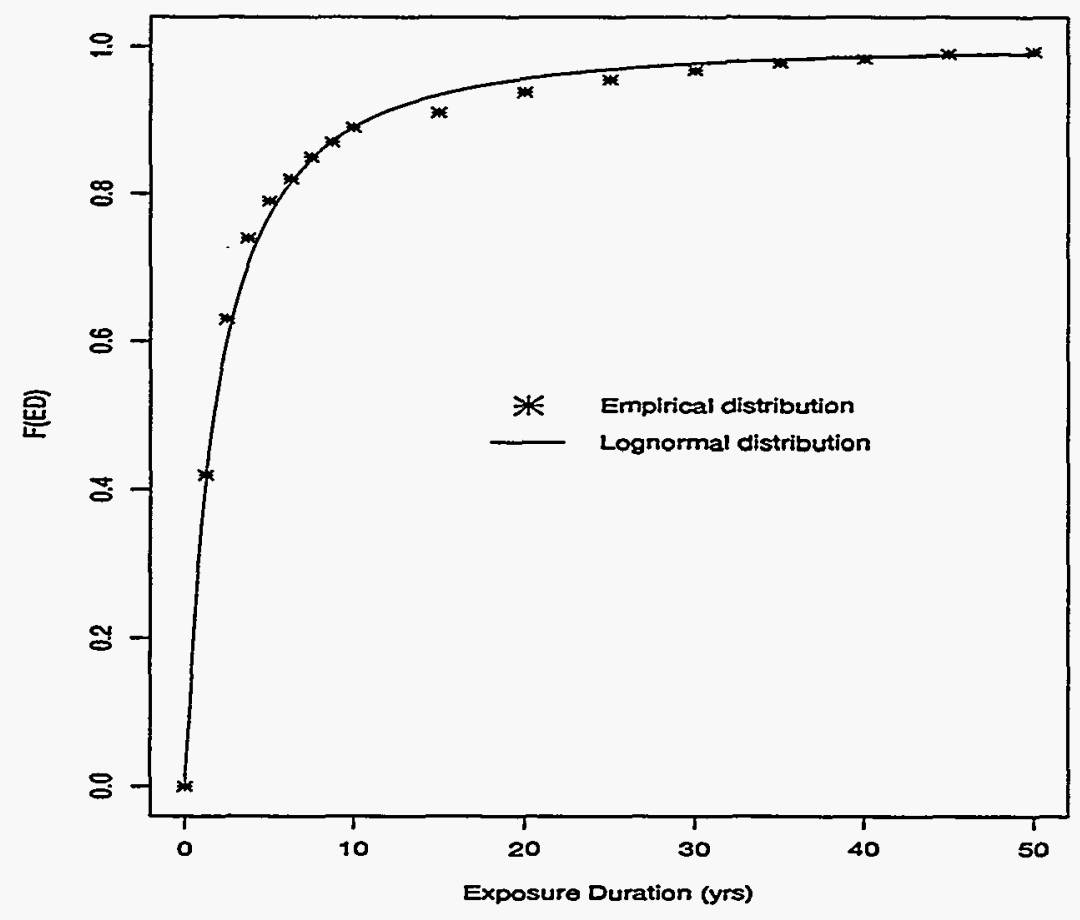

Figure 7. Exposure duration (total residence time) distribution fit. 


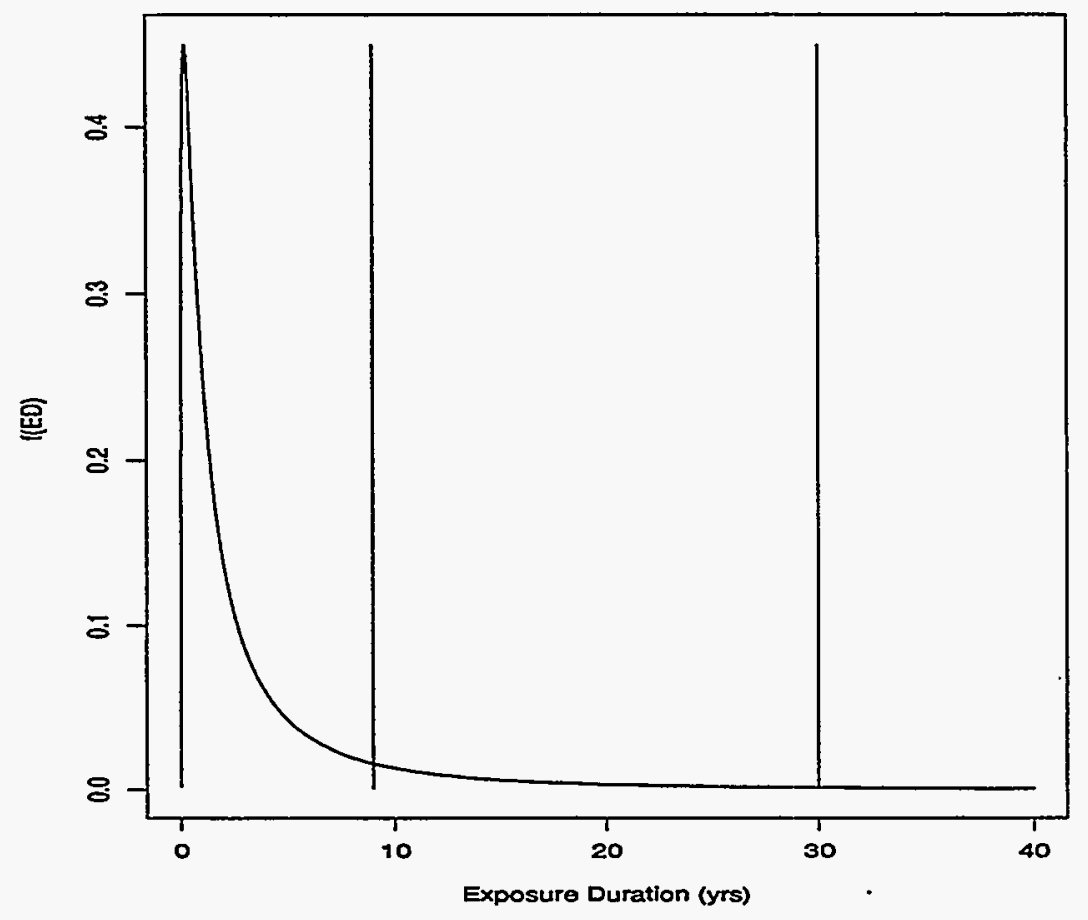

Figure 8. Probability density function for exposure duration. Vertical lines indicate the EPA point estimates of exposure duration at 9 and 30 years.

Brorby and Finley (1994) argue that the Calabrese study was of higher quality than other studies, including those cited by LaGoy (1987) and Thompson and Burmaster (1991) - though the latter do adjust their results for known study shortcomings. In any case, both Brorby and Finley (1994) and Thompson and Burmaster (1991) conclude that the distributional form for soil ingestion by children is lognormal.

If it is assumed that the adult ingestion rate is also lognormal, then it remains to estimate the mean and standard deviation of the distribution for use in the MCA. Brorby and Finley (1994) estimate the average ingestion of soil for children to be $21 \mathrm{mg} /$ day, which is less than the ingestion rate for adults as estimated by LaGoy at $25 \mathrm{mg} /$ day. The "intermediate" value of $10 \mathrm{mg} /$ day for adults cited by the EPA (1989b) does appear to be in agreement with the Brorby and Finley average, since adults are generally expected to ingest less soil than children. As such, an approximate mean of $10 \mathrm{mg} /$ day will be used for adults.
Selecting the standard deviation appears to be a bit trickier. The standard deviation of the underlying normal distribution reported by Thompson and Burmaster (1991) is 0.80 ; that reported by Brorby and Finley (1994) is $1.7(=\ln 5.5)$. Again, both of these numbers are for soil ingestion rates in children. Following the argument given in Brorby and Finley that the Calabrese data is of higher quality, there is reason to prefer their estimate of the standard deviation. Assuming that the relative variation in soil ingestion is roughly the same in adults and children, the standard deviation for the underlying normal distribution of 1.7 will be used.

Hence, a lognormal distribution with parameters (of the underlying normal distribution) mean 2 (which results in an arithmetic mean of the data of 10 in the original units, $\mathrm{mg} / \mathrm{d}$ ) and standard deviation 1.7 will be used. This distribution is plotted in Figure 9. The EPA recommended value of $100 \mathrm{mg} / \mathrm{d}$ falls at the 94 th percentile of this distribution, as shown in Figure 9. 


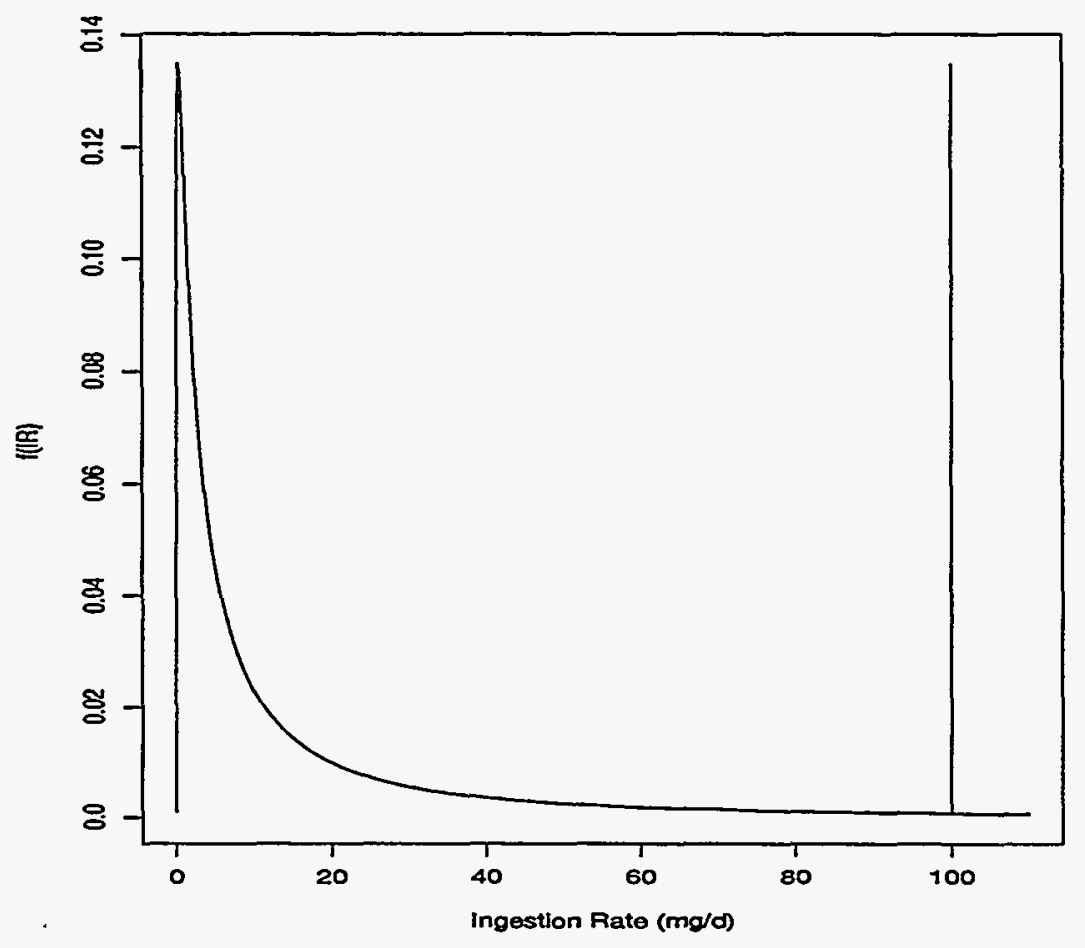

Figure 9. Probability density function for soil ingestion rate. Vertical line indicates the BRA point estimate of soil ingestion rate.

6.3.4 Slope Factor. The slope factor is the ratio of risk to unit dose. More specifically, the slopefactor represents an upper 95th percent confidence limit on the probability of a response per unit intake of a chemical over a lifetime. Since the slope factor is found by extrapolating from higher doses to low doses and is commonly based on experimental results with animals, it is subject to a great deal of uncertainty. That is, it is difficult if not impossible to observe a statistically meaningful estimate of the slope factor for low doses. Hence it is a modeled value that is generally not observable.

As previously discussed, there is some controversy as to whether slope factors, also known as cancer potency factors, should be considered probabilistically in an MCA analysis. The EPA (1994) suggests that the MCA should only be applied to exposure results, and not to toxicity results. The counter argument is that holding the slope factor constant in an MCA defeats its purpose since the slope factor is typically the most uncertain parameter.

Establishing the uncertainty in the slope factor requires a great deal of professional expertise. Lacking such expertise, one must rely heavily on available literature, if any. In this case, Thompson et al. (1992) have developed a CDF for ingestion of benzene. They found it to be lognormally distributed with the underlying normal distribution parameters of mean -4.33 and standard deviation 0.67. This distribution is shown in Figure 10. The verified EPA estimate for benzene ingestion, $2.9 \times 10^{-2}(\mathrm{mg} /(\mathrm{kg}-\mathrm{d}))^{-1}$, is shown in the Figure, and is located at approximately the 88 th percentile.

6.3.5 Averaging Time Distribution. The averaging time for carcinogens is the estimated lifetime over which the dose is averaged. Note that the units for the averaging time used in the equation given at the start of Section 6 are days. The discussion here uses units of years so that the reader can better understand the magnitude of the values. 


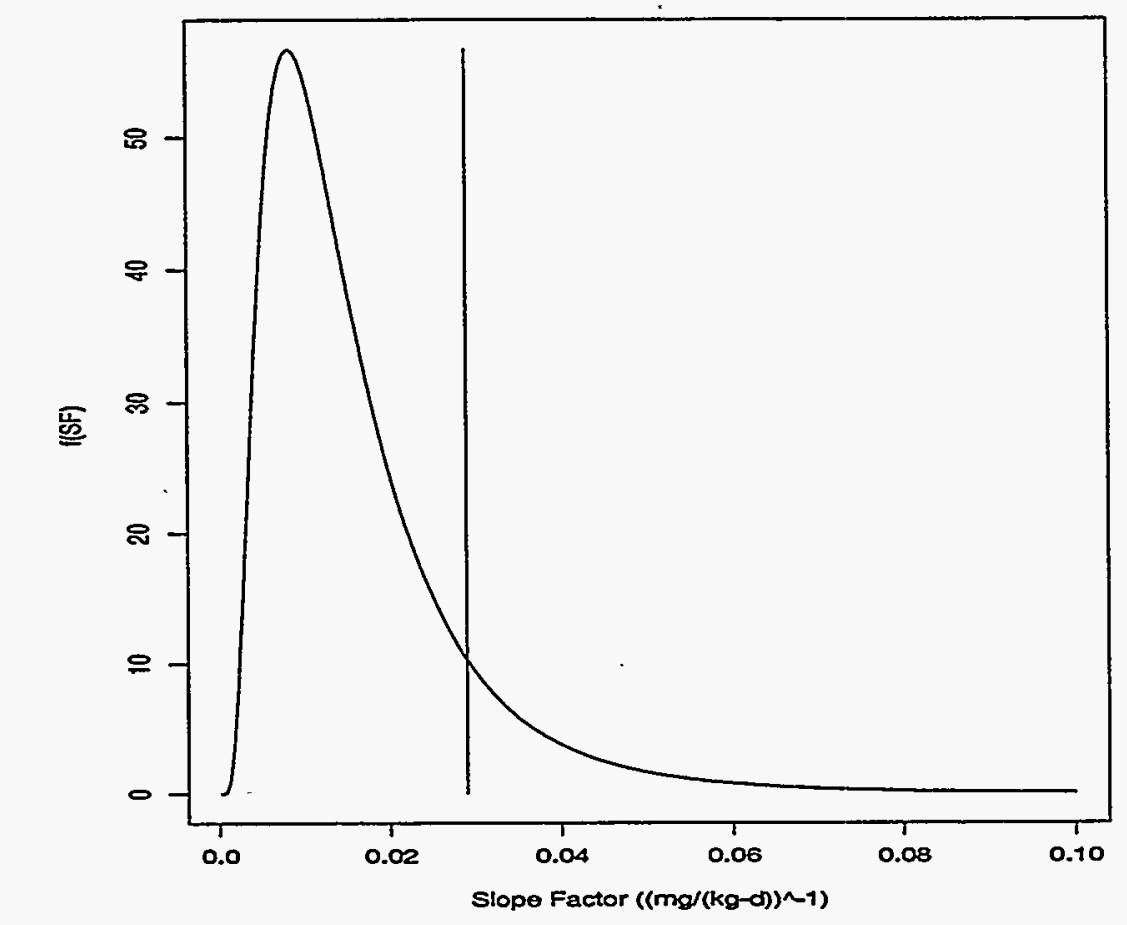

Figure 10. Probability density function for slope factor. The vertical line indicates EPA's estimated slope factor for benzene ingestion.

Using data contained in Wright (1993) and U.S. Bureau of the Census (1989), a lifetime distribution for persons 15 years and older was developed. Two types of distributions were fit to the data: Weibull and Beta. These were used because they easily accommodate negative skewness (i.e., leftskewed). The range used for the Beta distribution (which has a finite range, whereas the Weibull has an infinite range) was 15 to 100 years. Both distributions gave essentially the same fit (see Figure 11). The sum of the absolute deviations of the fitted value to the observed values was 0.0487 for the Beta distribution and 0.0481 for the Weibull distribution.

Both distributions result in median age at deaths of 73; this is about 5 years shorter than indicated from life expectancy information (Wright, 1993). This discrepancy is in part due to a lack of informa- tion on the death rates for ages greater than 85 , and not due to the fitting procedure. Using an age distribution with an expected value larger than the true value is not conservative for the averaging time parameter.

The Beta distribution, with shape parameters 4.2 and 2.1 will be used as the distribution in the Monte Carlo simulations. This is selected over the Weibull because the Weibull gives higher probabilities to extremely old ages. The Beta distribution cuts off at 100 years, which while it may not be realistic, is a bit more conservative, and may help to counteract the non-conservative expected value of the distribution. The PDF is shown in Figure 12. The EPA recommended value of 70 years is at the 42nd percentile of this distribution, as illustrated in Figure 12. 


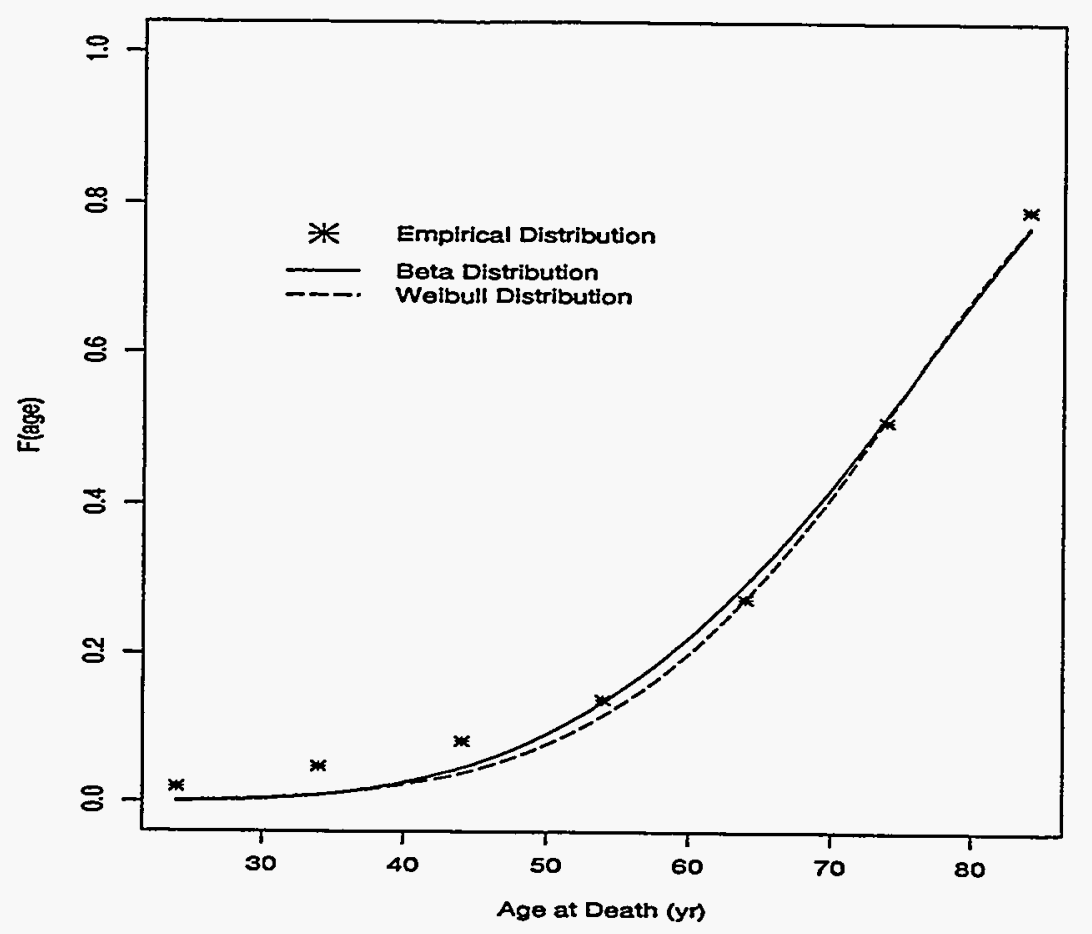

Figure 11. Averaging time (age) distribution fit. The size of the Kolmogorov-Smirnov indicates the vertical distance between the observed and fitted value that would be considered significant at $\alpha=0.10$.

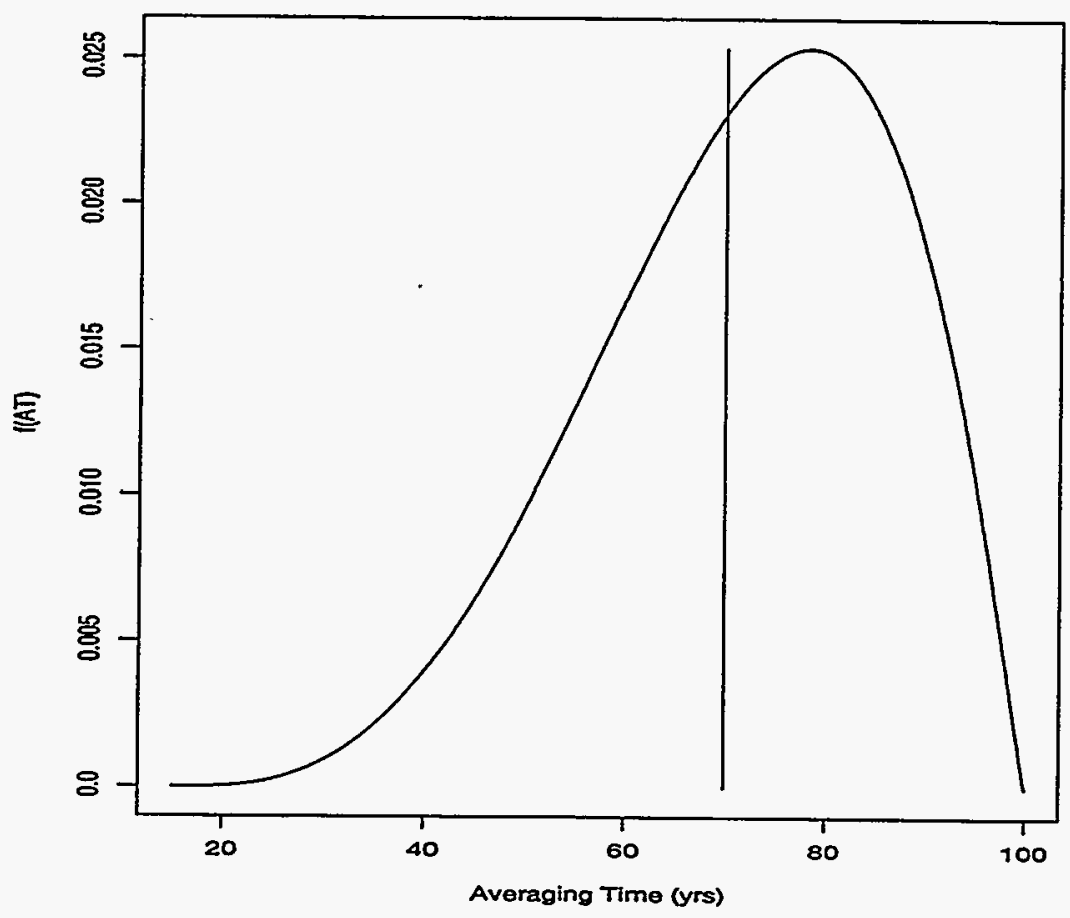

Figure 12. Probability density function for averaging time. Vertical line indicates the BRA point estimate. 
6.3.6 Body Weight. Body weight only has minor impact on the risk estimates. However, body weight distributions are readily available. One of the better quality studies is that by Brainard and Burmaster (1992). They find, not surprisingly, that the distribution differs for men and women. Men's weights are lognormally distributed with underlying normal parameters of mean 5.13 and standard deviation 0.17 . Women's weights are also lognormally distributed with underlying normal parameters of mean 4.96 and standard deviation 0.20 (original units are in $\mathrm{kg}$ ). These distributions are shown in Figure 13. For men, the EPA recommended value of $70 \mathrm{~kg}$ is at the 30 th percentile; for women, it is at the 65th percentile, as shown in Figure 13.

\subsection{Number of Monte Carlo Samples}

As discussed in Section 5.3.1, the number of Monte Carlo samples typically recommended is on the order of 10,000 . This number allows all percentiles of the resulting risk distribution to be estimated within one percentile with $95 \%$ confidence.
Since in this example a simple model is used to generate the exposures and risks, there is no need to consider LHS nor reducing the number of simulations. Hence, 10,000 simulations will be run. Further, a second set of 10,000 simulations will be run to validate the results from the first set of simulations.

\subsection{Monte Carlo Implementation}

The 10,000 sample run of the Monte Carlo simulation was implemented on a DECstation 5000/200 using S-Plus (Statistical Sciences, Inc., 1991). 10,000 randomly selected values were selected from each distribution shown in Table 5, except for body weight. For body weight, 5,000 samples were selected randomly from the male distribution and 5,000 from the female distribution. The parameters not treated probabilistically, exposure frequency and fraction ingested, were held at $340 \mathrm{~d} / \mathrm{yr}$ and 1.0 .

The results are summarized in Table 8 and the $\mathrm{CDF}$ and PDF of the risk are shown in Figures 14 and 15 , respectively. The expected risk was $1.2 \times 10^{-7}$ and the median was $1.8 \times 10^{-10}$. The

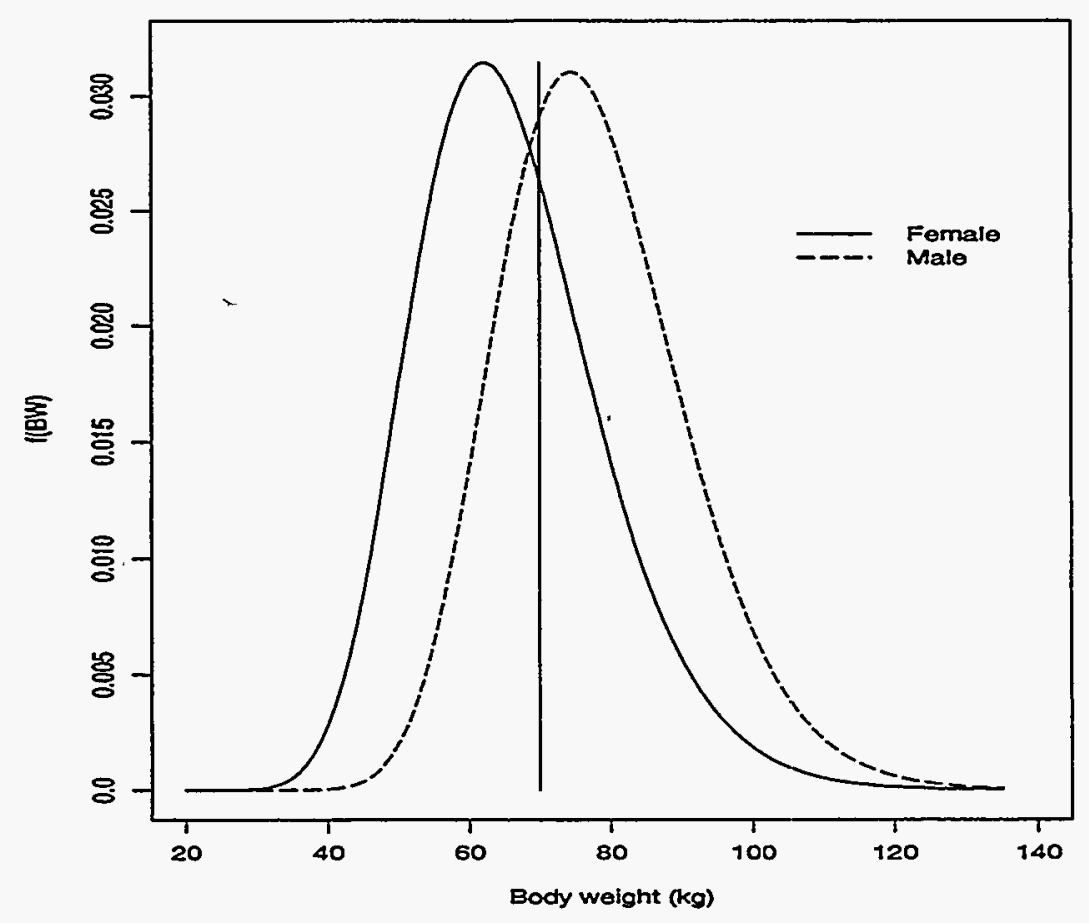

Figure 13. Probability density function for body weight. Vertical line indicates the BRA point estimate. 
Table 8. Summary statistics for results of the Monte Carlo analysis.

Statistics:

Mean $=1.2 \times 10^{-7} \quad$ Median $=1.8 \times 10^{-10} \quad$ Std. Dev. $=1.5 \times 10^{-5} \quad$ Maximum $=4.4 \times 10^{-4}$

Percentiles:

\begin{tabular}{cccccccc}
5 & $4.7 \times 10^{-13}$ & 30 & $2.8 \times 10^{-11}$ & 55 & $2.9 \times 10^{-10}$ & 80 & $3.8 \times 10^{-9}$ \\
10 & $1.8 \times 10^{-12}$ & 35 & $4.8 \times 10^{-11}$ & 60 & $4.5 \times 10^{-10}$ & 85 & $7.4 \times 10^{-9}$ \\
15 & $4.4 \times 10^{-12}$ & 40 & $7.4 \times 10^{-11}$ & 65 & $7.1 \times 10^{-10}$ & 90 & $1.7 \times 10^{-8}$ \\
20 & $9.0 \times 10^{-12}$ & 45 & $1.2 \times 10^{-10}$ & 70 & $1.2 \times 10^{-9}$ & 95 & $7.1 \times 10^{-8}$ \\
25 & $-1.7 \times 10^{-11}$ & 50 & $1.8 \times 10^{-10}$ & 75 & $2.1 \times 10^{-9}$ & 99 & $8.2 \times 10^{-7}$ \\
\hline
\end{tabular}

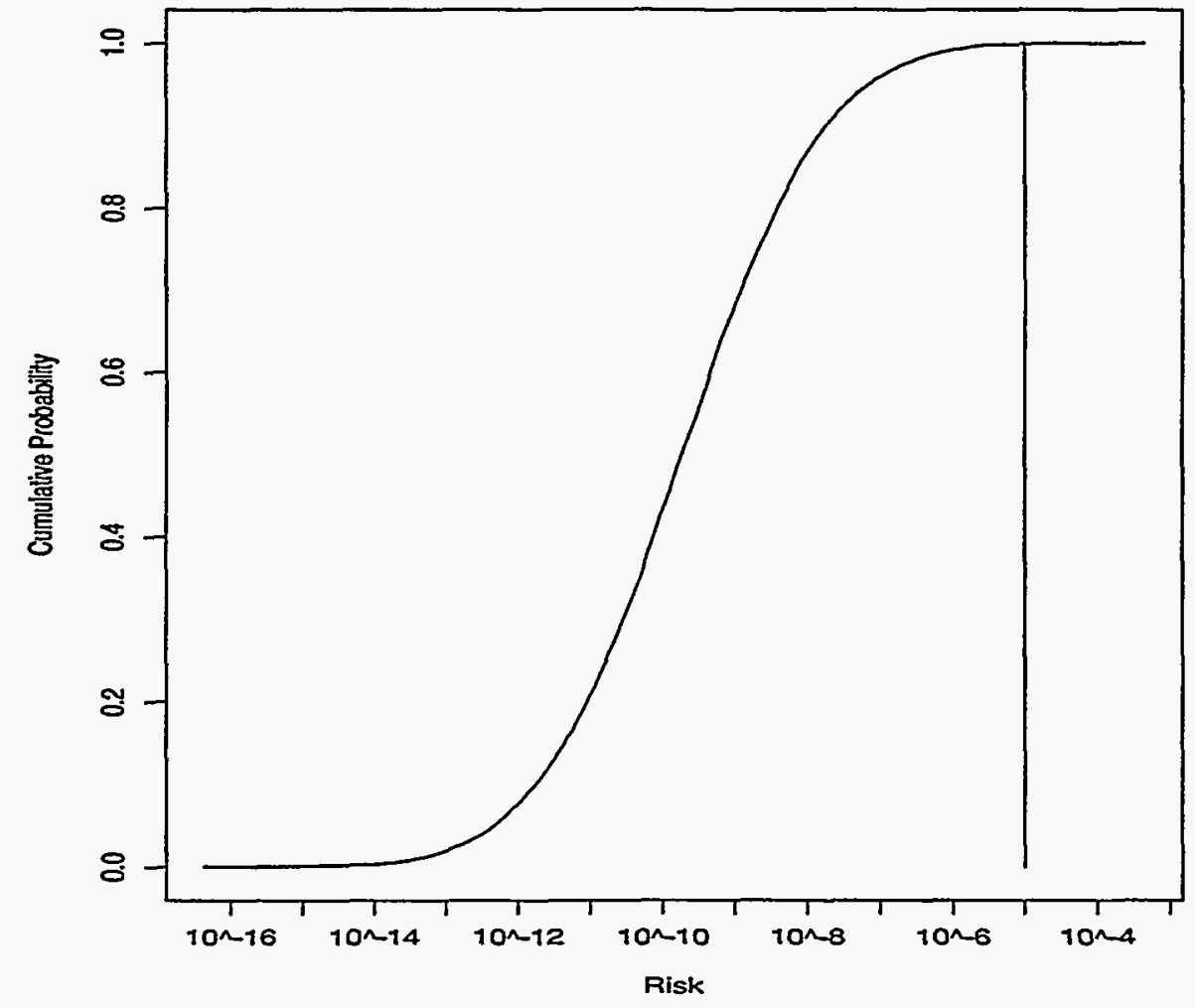

Figure 14. Cumulative distribution function of risks as observed from the Monte Carlo simulations. The vertical line indicates the baseline risk assessment point estimate. 


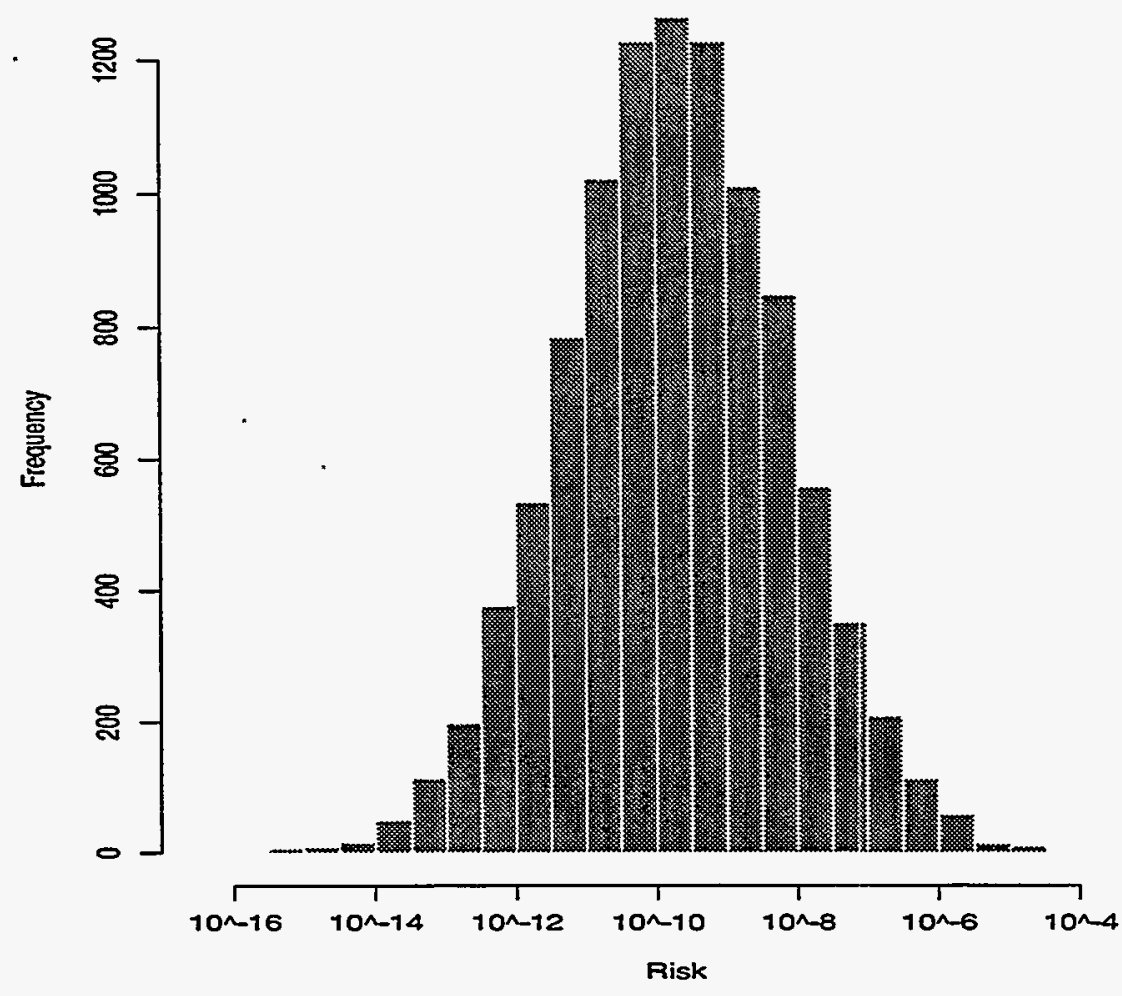

Figure 15. Probability density function of risks as observed from the Monte Carlo simulations. The BRA point estimate of the risk is $1 \times 10^{-5}$.

risks were approximately lognormally distributed with underlying normal parameters of mean -22.4 and standard deviation 3.6.

With this distribution estimation, the following can be concluded. There is roughly $50 \%$ probability that the risk for a given person exposed to benzene in soil will be less than $1.8 \times 10^{-10}$. There is a $0.9 \%$ probability that a given exposed person will have a risk greater than $1 \times 10^{-6}$, and less than a $0.03 \%$ chance that a given exposed individual will have a risk greater than $1 \times 10^{-4}$.

\subsection{Quality of the Results}

6.6.1 Reproducibility. To confirm the results of the initial simulations, a second set of 10,000 runs were made. While this may sound extreme, the actual time running the simulations and summarizing the results is small. On a DECstation
5000/200 using S-Plus, the simulations take under 5 seconds to run; calculating data summaries takes about a half-hour (including plots).

Overall, the replicate run was in substantial agreement with the original set of simulations. Comparison of the summary statistics are shown in Table 9. The mean, standard deviation, and quartiles, inclucling the median, show close agreement with each other. The maximum, which is more sensitive to skewness and outliers, is generally not reproducible. The lognormal mean estimates for the original and replicate samples are $1.2 \times 10^{-7}$ and $1.0 \times 10^{-7}$, respectively. The lognormal standard deviation estimates for the original and replicate samples are $1.5 \times 10^{-4}$ and $1.3 \times 10^{-4}$, respectively.

For the percentiles of the risk distribution shown in Table 10, the two sets of results again show close agreement through the center of the 
Table 9. Comparison of risk summary statistics between the original simulation and its replicate.

\begin{tabular}{lcccccc} 
Simulation & Mean $^{2}$ & Std. Dev. $^{\mathrm{a}}$ & 1st Quartile. & Median & 3rd Quartile & Maximum \\
\hline & & & & & & \\
Original & $1.2 \times 10^{-7}$ & $1.5 \times 10^{-4}$ & $1.7 \times 10^{-11}$ & $1.8 \times 10^{-10}$ & $2.1 \times 10^{-9}$ & $4.4 \times 10^{-4}$ \\
Replicate & $1.0 \times 10^{-7}$ & $1.3 \times 10^{-4}$ & $1.6 \times 10^{-11}$ & $1.8 \times 10^{-10}$ & $1.9 \times 10^{-9}$ & $4.4 \times 10^{-5}$
\end{tabular}

a. The mean and standard deviation are calculated using equations for lognormal distributions.

Table 10. Comparison of percentiles of the risk distributions between the original simulation and its replicate.

\begin{tabular}{ccccccccc} 
Simulation & \multicolumn{2}{c}{ Percentiles: } & & & & & \\
Original & 5 & $4.7 \times 10^{-13}$ & \multirow{2}{*}{30} & $2.8 \times 10^{-11}$ & 55 & $2.9 \times 10^{-10}$ & 80 & $3.8 \times 10^{-9}$ \\
Replicate & & $5.5 \times 10^{-13}$ & & $2.8 \times 10^{-11}$ &. & $2.7 \times 10^{-10}$ & & $3.6 \times 10^{-9}$ \\
Original & 10 & $1.8 \times 10^{-12}$ & \multirow{2}{*}{35} & $4.8 \times 10^{-11}$ & 60 & $4.5 \times 10^{-10}$ & 85 & $7.4 \times 10^{-9}$ \\
Replicate & & $1.8 \times 10^{-12}$ & & $4.5 \times 10^{-11}$ & & $4.4 \times 10^{-10}$ & & $6.8 \times 10^{-9}$ \\
Original & \multirow{2}{*}{15} & $4.4 \times 10^{-12}$ & \multirow{2}{*}{40} & $7.4 \times 10^{-11}$ & 65 & $7.1 \times 10^{-10}$ & 90 & $1.7 \times 10^{-8}$ \\
Replicate & & $4.3 \times 10^{-12}$ & & $7.3 \times 10^{-11}$ & & $7.0 \times 10^{-10}$ & & $1.6 \times 10^{-8}$ \\
Original & \multirow{2}{*}{20} & $9.0 \times 10^{-12}$ & \multirow{2}{*}{45} & $1.2 \times 10^{-10}$ & 70 & $1.2 \times 10^{-9}$ & 95 & $7.1 \times 10^{-8}$ \\
Replicate & & $8.4 \times 10^{-12}$ & & $1.2 \times 10^{-10}$ & & $1.2 \times 10^{-9}$ & & $7.0 \times 10^{-8}$ \\
Original & 25 & $1.7 \times 10^{-11}$ & \multirow{2}{*}{50} & $1.8 \times 10^{-10}$ & 75 & $2.1 \times 10^{-9}$ & 99 & $8.2 \times 10^{-7}$ \\
Replicate & & $1.6 \times 10^{-11}$ & & $1.8 \times 10^{-10}$ & & $1.9 \times 10^{-9}$ & & $7.3 \times 10^{-7}$ \\
\hline
\end{tabular}

distribution. At the 5th, 95th, and 99th percentile, there is some degradation of reproducibility. This increased variation in the tails is to be expected and reflects the uncertainty in the extreme risks.

So it can be concluded that 10,000 simulations provides reasonable precision in estimation for this example; while the precision at the high end of risk is not good. Certainly, using a larger number of simulations would improve the reproducibility of the high-end risk. However, because the largest uncertainty is associated with the tails of the input and resulting risk distributions, demanding better reproducibility is not likely to get one closer to the truth.

\subsubsection{Sensitivity of Risk to Input Parame-} ters. The final analysis is to assess the sensitivity of the uncertainty in the risk to each of the input parameters. Both the Pearson's correlation coefficient and Spearman's rank correlation coefficient were calculated to contrast the results; ordinarily only one of the correlations would be calculated. Recall from Section 5.3.2.2 that the Pearson's correlation coefficient is more sensitive to skewed distributions and outliers than Spearman's rank correlation coefficient. That is, in the presence of skewed data, the variation in the estimated Pearson's correlation coefficient will be larger than that in Spearman's coefficient.

The resulting correlations are shown in Table 11. The Pearson's correlation gives ambiguous results between the original and replicate simulations. However, the Spearman's correlation clearly indicates that benzene concentration in soil, soil 
Table 11. Measures of influence of the input parameters on the risk as observed in the original simulation and its replicate.

\begin{tabular}{llllllll}
\hline Correlation & Simulation & CS & IR & ED & BW & AT & SF \\
\hline \multirow{2}{*}{ Pearson's } & Original & 0.08 & 0.04 & 0.09 & 0.01 & 0.02 & 0.02 \\
& Replicate & 0.28 & 0.22 & 0.13 & 0.01 & 0.00 & 0.02 \\
& & & & & & & \\
Spearman's & Original & 0.75 & 0.46 & 0.36 & -0.06 & -0.07 & 0.20 \\
& Replicate & 0.74 & 0.48 & 0.37 & -0.05 & -0.06 & 0.17 \\
\hline
\end{tabular}

ingestion rate, exposure duration, and to a lesser extent slope factor have the largest impact on the uncertainty in the risk estimate. Hence if a risk risk, they would first attempt to reduce the uncertainties in these parameters, if possible. It is also interesting to note that the averaging time, which was very difficult to adequately fit a distribution to, had little impact on the risk; little information would have been lost had we held this parameter constant.

By comparing the Pearson and Spearman correlation results between the two sets of simulations, one sees the sensitivity of the Pearson correlation to skewness. For example, consider benzene concentration in soil (CS). The original simulation calculated a Pearson's correlation of 0.08 ; the replicate was 0.28 . For the same parameter, the Spearman's correlation was about 0.75 for both sets of simulations. These results clearly illustrate the advantage of a nonparametric correlation (e.g., Spearman's) over Pearson's correlation in the presence of skewed data.

\subsection{Conclusions}

The MCA results indicate that the probability that an individual is exposed to the BRA point estimate of risk is small, much less than $1 \%$. Additionally, there is only about a $1 \%$ probability that an individual will be subject to a risk greater than $1 \times 10^{-6}$. Individuals with high soil ingestion rates or residing at the location for a long period of time have the highest probability of being exposed to excessive risks.
The most important parameters were benzene concentration, ingestion rate, exposure duration, and slope factor. There was little data on adult soil ingestion rates, so the distribution used in the MCA was extrapolated from child ingestion rates (of which there is sufficient data). The exposure duration distribution used in the MCA is based on total residence times; these times were adjusted from the current residence times given by the EPA and others.

The slope factor was only the third most important input parameter; it generally would be expected to be of much greater importance. The distribution for slope factor was based on results given in Thompson et al (1992). They derived these distributions by adjusting the EPA recommended value for a variety of factors which are used to derive the slope factor. The resulting distributions may not have adequately accounted for all the uncertainty in this parameter.

The averaging time distribution, which was based on age at death, did not have a significant impact on risk. This distribution was slightly conservative; the median averaging time was about 73 years, while the true median is closer to 78 years. Shifting this distribution 5 yrs would not likely result in major changes to the calculated risks and particularly to the conclusions drawn. Also, the degree of conservatism in the distribution would be considered small.

In discussions with the risk manager, the risk analyst would emphasize the graphical results of the analysis as presented in Figures 14 and 15 . The analyst would indicate the best estimate is $99 \%$ of the 
population would be exposed to risks of less than $1 \times 10^{-6}$. The analyst would explain that the relatively high point estimates $\left(1 \times 10^{-5}\right)$ was mostly due to the use of the maximum benzene concentration in the soil. This occurred because of the great amount of variation in the observed benzene concentrations. 


\section{SUMMARY}

In this report, the motivation for using $\mathrm{MCA}$ as an improvement over the BRA point estimates is given. Two major shortcomings of the point estimate approach is that it suffers from creeping conservatism so that the user does not know how conservative the estimate is, and it provides limited information to the risk manager and public. The use of MCA helps alleviate both of these problems.

Some reasons for implementing an MCA include:

- Evidence shows that "best estimates" are not particularly good estimates; the quality of the estimates is significantly improved when the analysis forces the consideration of uncertainties.

- One purpose of risk assessment is to help anticipate the unexpected; again, by explicitly considering uncertainties, it is easier to determine the contingencies.

- $\quad$ Risk managers must rely on experts when making decisions; without uncertainty analysis, the manager cannot assess the reliability of the expert's information.

Some of the disadvantages of implementing an MCA include:

- Adds increased complexity to the risk assessment process
- Difficult to determine a good MCA from a bad MCA

- Some skepticism of MCA by EPA personnel.

Except under the situation in which the point estimate of risk leads to an unequivocal decision for an INEL site, the advantages of implementing an MCA far outweigh the disadvantages. A phased approach to implementing MCA risk analysis is recommended. Each phase would be a refinement, based on more information, of the previous phase. Sensitivity and correlation results from each phase would be useful in identifying which parameters to focus energies on.

While the actual implementation of MCA is straightforward, there are a number of issues that need to be agreed upon by all INEL stakeholders. These issues include selection of parameter distributions, use of expert judgment, implementation of correlation between parameters, and bounding of the assessment.

The use of MCA helps to focus attention on the uncertainty in the risk estimates. Further, it provides a quantitative description of the uncertainty. In this way, the manager and stakeholders are better able to understand the risks and are better able to make informed decisions. A key theme of the document is that uncertainty matters and $\mathrm{MCA}$ provides a practical description of the uncertainty. 


\section{REFERENCES}

Allen, Bruce C., Kenny S. Crump, and Annette M. Shipp (1988), "Correlation Between Carcinogenic Potency of Chemicals in Animals and Humans," Risk Analysis, 8(4):531-544.

Barry, Timothy M. (1993), "Monte Carlo Analysis in Support of Rulemaking at EPA-progress despite hurdles," a paper presented at the Workshop on the Application of Monte Carlo Modeling to Exposure and Risk Assessment, Savannah, GA, December 5, 1993.

Barry, Timothy M. (1994), "Monte Carlo Analysis Potpourri-Workshop on the Application of Monte Carlo Modeling to Exposure Assessment," presented at the Society for Risk Analysis Annual Meeting, Baltimore, MD, December 4, 1994.

Brainard, Jennifer and David E. Burmaster (1992), "Bivariate Distributions for Height and Weight of Men and Women in the United States," Risk Analysis, 12(2):267-275.

Brorby, Greg and Brent Finley (1993), "Standard Probability Density Functions for Routine Use in Environmental Health Risk Assessment," a paper presented at the Workshop on the Application of Monte Carlo Modeling to Exposure and Risk Assessment, Savannah, GA, December 5, 1993.

Burmaster, David E. and Paul D. Anderson (1994), "Principles of Good Practice for the Use of Monte Carlo Techniques in Human Health and Ecological Risk Assessments," Risk Analysis, 14(4):477-481.

Burmaster, David E. and Robert H. Harris (1993), "The Magnitude of Compounding Conservatisms in Superfund Risk Assessments," Risk Analysis, 13(2):131-134.

Calabrese, E.J., R. Barnes, E.J. Stanck, III, H. Pastides, C.E. Gilbert, P. Veneman, X. Wang, A. Laszity, and P.T. Kostecki (1989), "How Much Soil Do Young Children Ingest? An Epidemiologic Study," Regulatory Toxicology and Pharmacology, 10:123-137.

Cronin, William J., IV, Eric J. Oswald, Michael L. Shelley, Jeffrey W. Fisher, and Carlyle D. Flemming (1995), "A Trichloroethylene Risk Assessment Using a Monte Carlo Analysis of Parameter Uncertainty in Conjunction with Physiologically-Based Pharmacokinetic Modeling," Risk Analysis, 15(5):555-565.

Evans, Merran, Nicholas Hastings, and Brian Peacock (1993), Statistical Distributions, 2nd edition, New York: John Wiley \& Sons.

Finkel, Adam M. (1990), Confronting Uncertainty in Risk Management: A Guide for Decision-Makers, Center for Risk Management, Resources for the Future, Washington, D.C.

Finkel, Adam M. (1993), "Stepping Out of Your Own Shadow: A Didactic Example of How Uncertainty Can Inform and Improve Decision-Making," a paper presented at the Workshop on the Application of Monte Carlo Modeling to Exposure and Risk Assessment, December 5, 1993, Savannah, GA.

Finley, Brent, and Dennis Paustenbach (1994), "The Benefits of Probabilistic Exposure Assessment: Three Case Studies Involving Contaminated Air, Water, and Soil," Risk Analysis, 14(1):53-73.

Finley, Brent, Deborah Proctor, Paul Scott, Natalie Harrington, Dennis Paustenbach, and Paul Price (1994), "Recommended Distributions for Exposure Factors Frequently Used in Health Risk Assessment," Risk Analysis, 14(4):533-553. 
Freedman, David A., Lois Swirsky Gold, and Thomas H. Slone (1993), "How Tautological Are Interspecies Correlations of Carcinogenic Potencies?" Risk Analysis, 13(3):265-272.

Gilbert, Richard O. (1987), Statistical Methods for Environmental Pollution Monitoring, New York: Van Nostrand Reinhold, 1987.

Haimes, Yacov Y., T. Barry, and J.H. Lambert (eds.) (1994), "When and How Can You Specify a Probability Distribution When You Don't Know Much?," Risk Analysis, 14(5):661-706.

Hattis, Dale, and David E. Burmaster (1994), "Assessment of Variability and Uncertainty Distributions for Practical Risk Analyses," Risk Analysis, 14(5):713-730.

Hoffman, F.O. and J.S. Hammonds (1992), An Introductory Guide to Uncertainty Analysis in Environmental and Health Risk Assessment, ES/ER/TM-35, Oak Ridge National Laboratory.

Iman, Ronald L. and Jon C. Helton (1988), "An Investigation of Uncertainty and Sensitivity Analysis Techniques for Computer Modẹls," Risk Analysis, 8(1):71-90.

Israeli, Miron and Christopher B. Nelson (1992), "Distribution and Expected Time of Residence for U.S. Households," Risk Analysis, 12(1):65-72.

Johnson, N.L. and S. Kotz (1969), Distributions in Statistics: Discrete Distributions, John Wiley \& Sons, NY, NY.

Johnson, N.L. and S. Kotz (1970a), Distributions in Statistics: Continuous Univariate Distributions 1 , John Wiley \& Sons, NY, NY.

Johnson, N.L. and S. Kotz (1970b), Distributions in Statistics: Continuous Univariate Distributions 2, John Wiley \& Sons, NY, NY.

Johnson, N.L. and S. Kotz (1972), Distributions in Statistics: Continuous Multivariate Distributions, John Wiley \& Sons, NY, NY.

Kaplan, Stanley, and B. John Garrick (1981), "On the Quantitative Definition of Risk," Risk Analysis, $1(1): 11-27$.

Knuth, Donald E. (1969), The Art of Computer Programming, Volume 2/Seminumerical Algorithms, Addison Wesley Publishing Co, Reading, MA.

LaGoy, Peter K. (1987), "Estimated Soil Ingestion Rates for Use in Risk Assessment," Risk Analysis, $7(3): 355-359$.

Lambert, James H., Nocholas C. Matalas, Con Way Ling, Yocov Y. Haimes, and Duan Li (1994), "Selection of Probability Distributions in Characterizing Risk of Extreme Events," Risk Analysis, 14(5):731-742.

McKone, Thomas E. and Kenneth T. Bogen (1991), "Predicting the uncertainties in risk assessment," Environmental Science and Technology, 25(10):1674-1681.

Morgan, M. Granger, and Max Henrion (1990), Uncertainty: A Guide to Dealing with Uncertainty in Quantitative Risk and Policy Analysis, New York: Cambridge University Press, 1990. 
Price, Paul (1993a), "Uncertainty and Variation in Regulatory Toxicology," a paper presented at the Workshop on the Application of Monte Carlo Modeling to Exposure and Risk Assessment, Savannah, GA, December 5, 1993.

Price, Paul (1993b), "Monte Carlo Case Example: Exposure to Dioxins from the Consumption of Fish Caught Downstream of Pulp and Paper Mill Discharges," a paper presented at the Workshop on the Application of Monte Carlo Modeling to Exposure and Risk Assessment, Savannah, GA, December 5 , 1993.

Price, Paul S., James Sample, and Robert Strieter (1992), "Determinations of Less-Than-Lifetime Exposures to Point Source Emissions," Risk Analysis, 12(3):367-382.

Rish, William R. (1988), Approach to Uncertainty in Risk Analysis, ORNL/TM-10746, Oak Ridge National Lab.

Seiler, Fritz A. and Joseph L. Alvarez (1996), "On the Selection of Distributions for Stochastic Variables," Risk Analysis, 16(1):5-18.

Smith, Andrew E., P. Barry Ryan, and John S. Evans (1992), "The Effect of Neglecting Correlations When Propagating Uncertainty and Estimating the Population Distribution of Risk," Risk Analysis, 12(4):467-474.

Smith, T.H. (1994), "Parameters Expected to be Sensitive and Uncertain in Probabilistic Risk Assessments of INEL Environmental Restoration Sites," Draft White Paper, LITCO.

Steel, Robert G. D. and James H. Torrie (1980), Principles and Procedures of Statistics: A Biometrical Approach, 2nd edition, New York: McGraw-Hill Book Co.

Thompson, Kimberly M. and David E. Burmaster (1991), "Parametric Distributions for Soil Ingestion by Children," Risk Analysis, 11(2):339-342.

Thompson, Kimberly M., David E. Burmaster, and Edmund A.C. Crouch (1992), "Monte Carlo Techniques for Quantitative Uncertainty Analysis in Public Health Risk Assessments," Risk Analysis, 12(1):53-63.

U.S. Bureau of the Census (1989), Statistical Abstract of the United States: 1989 (109th edition.), Washington, DC.

U.S. Department of Energy (1994a), Information Brief on Risk Characterization: EPA Guidance on Risk Characterization For Risk Managers and Risk Assessors, EH-232-0008/08/94.

U.S. Department of Energy (1994b), Information Brief on Guidelines for Exposure Assessment: Exposure Assessment Guidelins, EH-232-0009/08/94.

U.S. Environmental Protection Agency (1989a), Risk Assessment Guidance for Superfund: Volume 1-Human Health Evaluation Manual (Part A), EPA/540/1-89/002.

U.S. Environmental Protection Agency (1989b), Exposure Factors Handbook, EPA/600/8-89/043.

U.S. Environmental Protection Agency (1989c), Interim Final Guidance for Soil Ingestion Rates, OSWER Directive 9850.4. 
U.S. Environmental Protection Agency (1991), Health Effects Assessment Summary Tables, PB91-921100.

U.S. Environmental Protection Agency (1992a), Guidelines for Exposure Assessment: Notice, May 29, 1992, 57(104) FR 22888, Washington, D.C.

U.S. Environmental Protection Agency (1992b), Supplemental Guidance to RAGS: Calculating the Concentration Term, OSWER Publication 9285.7-081.

U.S. Environmental Protection Agency (1993), An SAB Report: Superfund Site Health Risk Assessment Guidelines; Review of the Office of Solid Waste and emergency Responses Draft Risk Assessment Guidance for Superfund Human Health Evaluation Manual by the Environmental Health Committee, EPA-SAB-EHC-93-007.

U.S. Environmental Protection Agency (1994), "Use of Monte Carlo Simulation in Risk Assessments," Region III Technical Guidance Manual, EPA 903-F-94-001.

Wright, John W., editor (1993), The Universal Almanac 1994, Andrews and McMeel, Kansas City, MO. 